\title{
Vergleichende MR-volumetrische Untersuchung des dorsolateralen präfrontalen Kortex bei Schizophrenie, Bipolarer Störung, Zwangserkrankung und gesunden Kontrollpersonen
}

\author{
INAUGURAL-DISSERTATION \\ zur Erlangung des Doktorgrades \\ für Zahnheilkunde \\ der Medizinischen Fakultät \\ der Georg-August-Universität zu Göttingen \\ vorgelegt von \\ Kristina Kremer
}

aus

Göttingen

Göttingen 2010 
Dekan:
I. Berichterstatter:
Priv.-Doz. Dr. med. T. Wobrock
II. Berichterstatter:
III. Berichterstatter:
Priv.-Doz. Dr. rer. nat. P. Dechent
Priv.-Doz. Dr. med. J. Schmidt

Tag der mündlichen Prüfung: 11.04.2011
Prof. Dr. med. C. Frömmel 


\section{Inhaltsverzeichnis}

Abkürzungsverzeichnis

1. Einleitung. 1

1.1. Allgemeines, Epidemiologie und Ätiopathogenese der Schizophrenie 2

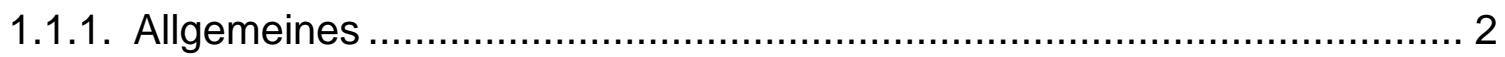

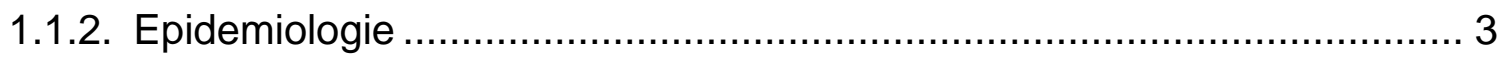

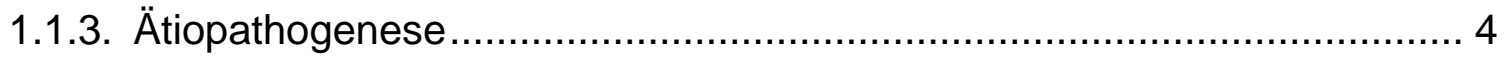

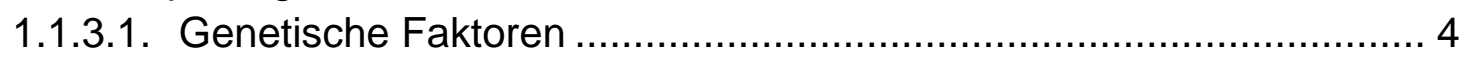

1.1.3.2. Hirnstrukturelle Befunde .................................................. 5

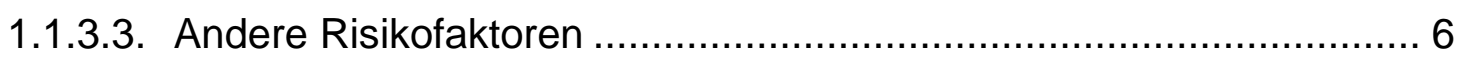

1.2. Hirnvolumetrische Messungen bei Schizophrenie................................ 8

1.3. Dorsolateraler präfrontaler Kortex - Morphologie und Funktion ........... 9

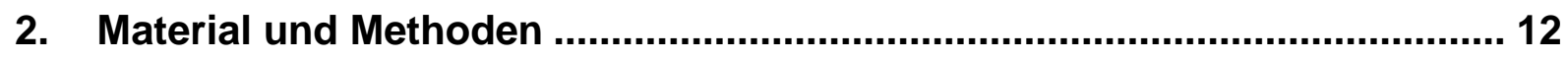

2.1. Fragestellung der Arbeit ............................................................. 12

2.2. Beschreibung der verwendeten psychopathologischen Skalen.......... 12

2.3. Klinische und soziodemographische Daten der Patientengruppen .... 14

2.3.1. Deskriptive Statistik der Gruppe 'schizophrene Patienten' ................... 14

2.3.2. Deskriptive Statistik der Gruppe 'Bipolare Patienten' ........................... 17

2.3.3. Deskriptive Statistik der Gruppe 'Zwangspatienten' ............................ 17

2.3.4. Deskriptive Statistik der Gruppe 'gesunde Kontrollen' ........................ 18

2.4. MR-technische Parameter und Volumetrie ...................................... 19

2.5. Definition der anatomischen Grenzen des DLPFC............................ 21

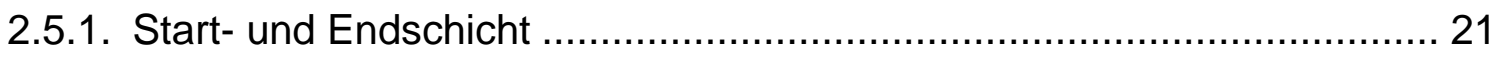

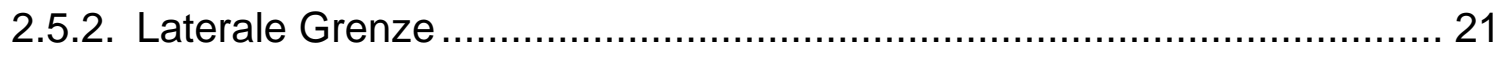

2.5.3. Mediale Grenze ............................................................... 22

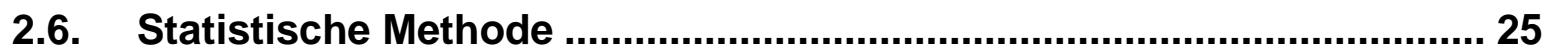

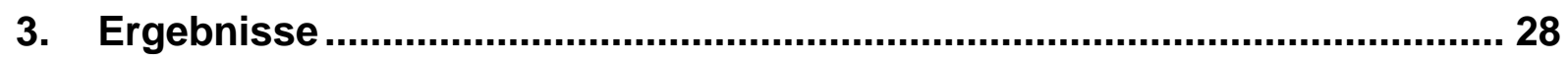

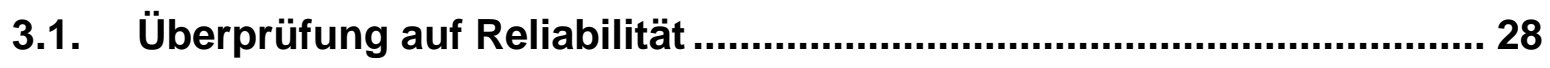

3.2. Soziodemografische und klinische Variablen der untersuchten MRStichproben im Vergleich ................................................................ 29

3.3. Vergleich der DLPFC-Volumina zwischen den Diagnosegruppen ....... 33

3.4. Berücksichtigung des Einflusses intervenierender Variablen ............ 36

3.5. Einfluss intervenierender Variablen bei Schizophrenie...................... 40

3.5.1. Einfluss familiärer Belastung bei schizophren Ersterkrankten ..............42 42 
3.5.2. Einfluss des Krankheitsverlaufs und der Psychopathologie bei schizophren Ersterkrankten .

3.6. Einfluss intervenierender Variablen bei Bipolaren Patienten ............. 47

3.6.1. Einfluss des Krankheitsverlaufs bei Bipolaren Patienten ....................... 47

3.7. Einfluss intervenierender Variablen bei Zwangspatienten................... 47

3.7.1. Einfluss des Krankheitsverlaufs bei Zwangspatienten .......................... 48

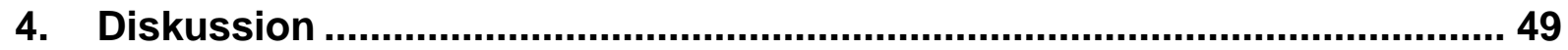

4.1. Kritische Wertung methodischer Aspekte ....................................... 54

4.2. Bewertung des Einflusses intervenierender Variablen........................62

4.3. Bewertung der Subgruppenvergleiche ..............................................64

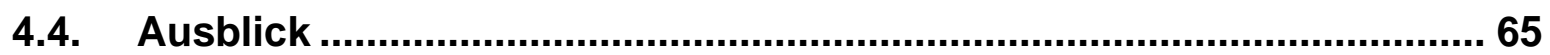

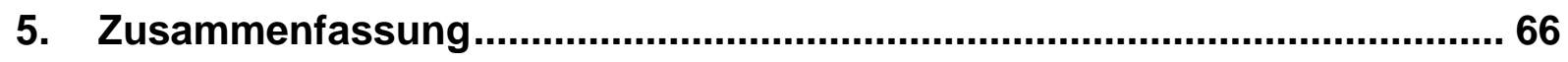

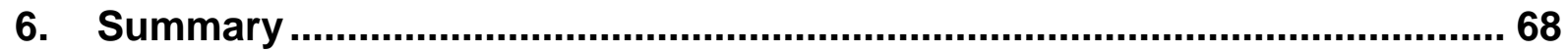

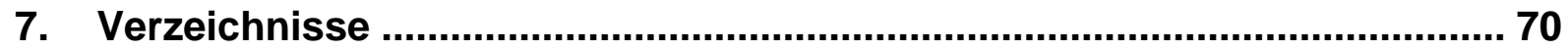

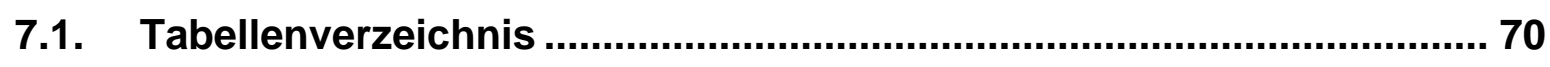

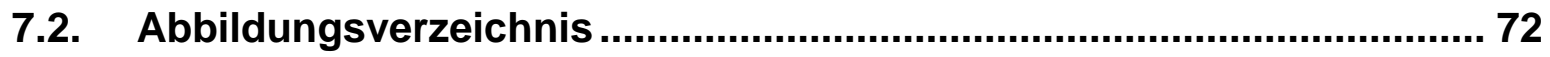

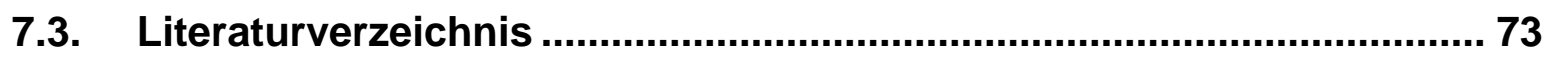




\section{Abkürzungsverzeichnis}

\begin{tabular}{|c|c|}
\hline a & Alter \\
\hline Abb. & Abbildung \\
\hline Al & Asymmetriekoeffizient \\
\hline ANCOVA & analysis of covariance \\
\hline ANOVA & analysis of variance \\
\hline $\mathrm{b}$ & Bildung \\
\hline Bip. & Bipolare \\
\hline C & Kontrollen \\
\hline CS & chronisch Schizophrene \\
\hline CGI & Clinical Global Impressions (Guy 1976/2000) \\
\hline $\mathrm{CPZ}$ & Chlorpromazin-Äquivalente \\
\hline df & Freiheitsgrade \\
\hline DLPFC & dorsolateraler präfrontaler Kortex \\
\hline DSM IV & $\begin{array}{l}\text { Diagnostic and Statistical Manual of Mental Disorders IV (Saß et } \\
\text { al. 1996) }\end{array}$ \\
\hline DUI & Dauer unbehandelte Erkrankung \\
\hline DUP & Dauer unbehandelte Psychose \\
\hline $\mathrm{F}$ & Statistik \\
\hline FENS & first-episode not schizophrenia \\
\hline FES & first-episode schizophrenia \\
\hline g & Geschlecht \\
\hline GAF & Global Assessment of Functioning \\
\hline GS & graue Substanz \\
\hline $\mathrm{h}$ & Händigkeit \\
\hline ICC & Intraclasscorrelation \\
\hline ICD & $\begin{array}{l}\text { International Statistical Classification of Diseases and Related } \\
\text { Health Problems (Dilling et al. 1999) }\end{array}$ \\
\hline I & links \\
\hline $\mathrm{m}$ & Mittelwert \\
\hline MADRS & Montgomery-Asberg depression rating scale \\
\hline MANCOVA & multiple analysis of covariance \\
\hline MANOVA & multivariate analysis of variance \\
\hline MRT & Magnetresonanztomographie \\
\hline $\mathrm{ms}$ & Millisekunde \\
\hline $\mathrm{n}$ & Fallzahl \\
\hline OCD & Obsessive-compulsive disorder \\
\hline $\mathrm{p}$ & Wahrscheinlichkeit für Fehler erster Ordnung \\
\hline p.c. & partielle Korrelation \\
\hline PANSS & Positive and Negative Syndrome Scale \\
\hline
\end{tabular}




$\begin{array}{ll}\text { PFC } & \text { präfrontaler Kortex } \\ r & \text { rechts } \\ \text { ROI } & \text { region of interest } \\ \text { sd } & \text { Standardabweichung } \\ \text { SPM } & \text { Statistical Parametric Mapping } \\ \text { Sz } & \text { Schizophrene Patienten } \\ \text { V } & \text { Volumen } \\ \text { VBM } & \text { voxel-basierte Morphometrie } \\ \text { VOI } & \text { volume of interest } \\ \text { WHO } & \text { world health organisation } \\ \text { WS } & \text { weiße Substanz } \\ \text { YMRS } & \text { young mania rating scale (score) }\end{array}$




\section{$1 \quad$ Einleitung}

Die Schizophrenie ist eine Hirnerkrankung, bei der es zu einer Störung in zahlreichen Bereichen des seelischen Erlebens wie des inhaltlichen und formalen Denkvermögens, der Wahrnehmung, des Icherlebens, der Affektivität, des Antriebs, der Psychomotorik, der Aufmerksamkeit, der Konzentration und des Gedächtnisses kommt. Die Lebenszeitprävalenz liegt weltweit zwischen 0,5 und 1,6\%. Die Erkrankung tritt bevorzugt zwischen dem 15. und dem 35. Lebensjahr auf. Als Kardinalsymptome werden das Vorhandensein von Halluzinationen, Wahnvorstellungen, von anderen Personen kontrolliert zu werden, emotionaler Verflachung und fehlender Krankheitseinsicht angesehen (Falkai et al. 2002).

Genetische sowie umweltbedingte Faktoren wirken zusammen. Das "VulnerabilitätsStress-Bewältigungs-Modell" gibt die vielschichtige Ätiopathogenese derzeit am besten wieder (Nuechterlein et al. 1994). Ätiopathogenetisch ist bei der schizophrenen Erkrankung davon auszugehen, dass zumindest bei einer Subgruppe der Patienten eine komplexe Störung der Neuro- und Myeloarchitektonik vorliegt. Weiterhin wird davon ausgegangen, dass die synaptischen Umbauvorgänge in mehreren Hirnregionen mit Betonung der frontalen, temporalen und limbischen Strukturen gestört sind. Dies führt zu einer beeinträchtigten Konnektivität von neuronalen Netzwerkverbänden. Neurobiochemisch wird eine Störung des Neurotransmittergleichgewichtes angenommen, welche neben einer dopaminergen Überfunktion des mesolimbischen Systems auch Veränderungen anderer Systeme wie Glutamat, Serotonin und GABA ( $\gamma$-Aminobuttersäure) beinhaltet.

Die bis dato verwendeten medikamentösen Therapieansätze (Antipsychotika) basieren alle auf dem Prinzip der Blockade von Dopamin D2-Rezeptoren, trotz in den letzen Jahren vielfältiger anderer Überlegungen, wie beispielsweise die Modulation des glutamatergen Systems oder des Endocannabinoidsystems. Ein besseres Verständnis der pathophysiologischen Grundlagen der Erkrankung, zu dem auch volumetrische Untersuchungen von Schlüsselregionen wie des frontalen Kortex beitragen, könnte zu innovativen und mehr kausal orientierten Therapieansätzen führen. 


\subsection{Allgemeines, Epidemiologie und Ätiopathogenese der Schizophrenie}

\subsubsection{Allgemeines}

Die Krankheitsbezeichnung Schizophrenie geht auf E. Bleuler (Bleuler 1911) zurück, der darunter eine Aufspaltung des Denkens, Fühlens und Wollens sowie der des subjektiven Gefühls der Persönlichkeit verstand. Im Gegensatz zu E. Kraepelin (Kraepelin 1896), der die Dementia praecox mit stets ungünstiger Prognose dem manisch-depressiven Irresein gegenüberstellte, beschrieb Bleuler mit seiner Konzeption der Gruppe der Schizophrenien bereits ätiologische und prognostische Heterogenität der Erkrankung.

In der Internationalen Erkrankungsklassifikation der WHO, der ICD-10 (Dilling et al. 1999), wird die Symptomatologie mit einer grundlegenden Persönlichkeitsveränderung, durch charakteristische Verzerrungen von Denken und Wahrnehmung sowie unpassende oder abgeflachte Affekte, beschrieben. Die Klarheit des Bewusstseins ist in der Regel nicht beeinträchtigt. Zusammengefasst werden aufgrund ihrer ähnlichen psychopathologischen Symptome in Kapitel V(F), in Rubrik F2, die Schizophrenie, schizotype und wahnhafte Störungen (Dilling et al.1999).

Obwohl im triadischen System die Schizophrenie eine Hauptgruppe der endogenen Psychosen darstellt und die Schizophrenie als funktionelle Störung den hirnorganischen Erkrankungen gegenüber gestellt wird, werden im Zuge der Erforschung der neurobiologischen Grundlagen dieser Erkrankung zunehmend subtile hirnstrukturelle Veränderungen bei an Schizophrenie Erkrankten gefunden (Falkai und Bogerts 1986).

Laut Schmitt et al. (2001) konnte durch die nichtinvasiven morphologischen Bildgebungsverfahren (Computertomographie, Kernspintomographie, MRT) wesentlich zu der Erkenntnis beigetragen werden, dass die Schizophrenien Erkrankungen des Gehirns sind.

Die Schizophrenie tritt in allen Völkern und Kulturen gleich häufig auf (Häfner 1988). Ausprägung und Häufung sind in einfacheren Wohngebieten von Großstädten und generell in städtischen gegenüber ländlichen Regionen ausgeprägter (Falkai et al. 2002). Sie zählt zu den zehn am häufigsten zur Behinderung (z.B. gemessen in 
„Disability Adjusted Life Years“) führenden Erkrankungen im Alter von 15-44 Jahren. Damit kommt der Weiterentwicklung effizienter Behandlungsstrategien für dieses Krankheitsbild aus ätiopathogenetischen Überlegungen heraus mit der Konsequenz einer mehr ursachenorientierten Therapie eine enorme Bedeutung zu (Wobrock et al. 2004). Da sie in der Regel junge Erwachsene betrifft und bei ca. der Hälfte von ihnen zu einer deutlichen Beeinträchtigung der psychosozialen Entwicklungsfähigkeit, der Erreichung normaler beruflicher wie auch privater Zielsetzungen, führt, zählt sie aufgrund ihrer indirekten Folgekosten zu den zehn kostenintensivsten Krankheiten weltweit (Falkai et al. 2002).

\subsubsection{Epidemiologie}

Die Lebenszeitprävalenz, d.h. das Risiko einer bestimmten Person, im Laufe des Lebens mindestens einmal an einer schizophrenen Episode zu erkranken, liegt weltweit zwischen 0,5 und 1,6\%. Die Anzahl der Ersterkrankungen im Hauptrisikoalter (15-59 Jahre) (Inzidenzrate) liegt zwischen 0,16 und 0,42 pro 1000 Einwohner und die Erkrankungshäufigkeit zu einem bestimmten Zeitraum in einer definierten Bevölkerung schwankt zwischen 1,4 und 4,6 pro 1000 Einwohner (Punktprävalenz) (Jablensky 2000). Die Erkrankung tritt bevorzugt zwischen dem 15. und dem 35. Lebensjahr auf. Das Lebenszeitrisiko zwischen den Geschlechtern ist gleich, Männer erkranken jedoch etwa 3-4 Jahre früher als Frauen, wobei als Ursache psychosoziale, subtypologische und neurohumorale Faktoren wie eine Schutzwirkung des Östrogens diskutiert werden (Häfner et al. 1993). Unter Personen mit niedrigem Bildungsabschluss und niedrigem sozioökonomischem Status sind schizophrene Psychosen gehäuft zu finden, was darauf zurückgeführt wird, dass es durch die Krankheit selbst bereits früh (teilweise schon im Vorstadium der Krankheit) zu einer Beeinträchtigung des sozialen Status kommen kann.

Die Mortalitätsrate ist bei an Schizophrenie Erkrankten im Vergleich zur gesunden Bevölkerung mit 10\% deutlich erhöht. Diese geht auf unnatürliche Todesursachen, wie den Selbstmord, aber auch auf ein z.B. erhöhtes kardiovaskuläres Risiko zurück (Falkai et al. 2002).

In Deutschland befinden sich etwa 97\% aller Kranken mit Schizophrenie in ambulanter Behandlung, nur ca. $3 \%$ in einer stationären Langzeitbehandlung 
(Bestehehorn et al.1999). Die direkten und indirekten Kosten sind denen somatischer Volkskrankheiten mindestens vergleichbar und werden pro Jahr in Deutschland bei ca. 400.000 erkrankten Patienten auf 4 bis 9 Milliarden Euro geschätzt (Kissling et al. 1999). Die Hälfte aller psychiatrischen Versorgungsleistungen in vielen Ländern muss für Patienten mit einer Schizophrenie verwandt werden.

\subsection{3 Ätiopathogenese}

Die Schizophrenie ist eine komplexe Erkrankung, bei der die Ursachen noch weitgehend unbekannt sind. Das "Vulnerabilitäts-Stress-Bewältigungs-Modell" (Nuechterlein et al. 1994) geht von einer permanent, d.h. auch im interepisodischen Intervall vorhandenen subklinischen - neuropsychologisch und psychophysiologisch nachweisbaren Vulnerabilität im Sinne einer Disposition für die Manifestation einer Schizophrenie aus. Die Ursache wird in genetischen und/oder nicht-genetischen Einflüssen (z.B. Geburtskomplikationen) gesehen. Genetische Faktoren können zu ca. $50 \%$ die Basisvulnerabilität erklären. Schwangerschafts- und Geburtskomplikationen erhöhen das Risiko, an einer Schizophrenie zu erkranken, um ca. weitere 1-2\%. In der Kindheit können zusätzliche Faktoren wie z.B. der frühe Verlust eines Elternteils, körperliche Misshandlung oder sexueller Missbrauch das Risiko für eine spätere psychische Störung erhöhen. Endogene und exogene Stressoren biologischer und psychosozialer Natur führen dann bei reduzierter Verarbeitungskapazität und nicht ausreichenden Bewältigungsstrategien zu einem passageren Funktionsversagen mit der klinischen Konsequenz einer akuten psychotischen Symptomatik.

\subsubsection{Genetische Faktoren}

Die schizophrene Erkrankung gehört zu den genetisch komplexen Erkrankungen, bei denen mittels Kopplungs- und Assoziationsuntersuchungen eine Reihe von Suszeptibilitätsgenen (Kandidatengenen) identifiziert werden konnten. Die einzelnen Gene liefern jeweils nur einen geringen Beitrag zur Gesamtvulnerabilität (Maier et al. 1999). Belegt wird die genetische Komponente (polygener Erbgang) durch Familien-, 
Adoptions- und Zwillingstudien. Im Vergleich von eineiigen und zweieiigen Zwillingspaaren wurde ein mehr als doppelt so häufiges Vorkommen einer Schizophrenie bei den monozygoten Zwillingen beobachtet (Cardno und Gottesmann 2000).

Das Lebenszeitrisiko für Angehörige ersten Grades, ebenfalls an einer schizophrenen Psychose zu erkranken, liegt ca. 10-15 fach höher als das der übrigen Bevölkerung und ist etwa so hoch wie das Erkrankungsrisiko adoptierter so genannter High-Risk-Kinder (Gottesmann und Bertelsen 1989).

Genetisch vermittelte Anlagen zur Entwicklung einer Schizophrenie können sich auch in geringer Ausprägung manifestieren, so dass lediglich die Kriterien der schizophrenen Spektrumerkrankung erfüllt werden.

\subsubsection{Hirnstrukturelle Befunde}

Es ist davon auszugehen, dass bei der Schizophrenie eine komplexe Störung der Neuro- und Myeloarchitektonik einschließlich der synaptischen Umbauvorgänge mit Betonung der fronto-temporalen Strukturen vorliegt, die zu einer fokalen Dysfunktion und einer beeinträchtigten Konnektivität des zugrundeliegenden neuronalen Netzwerkes führt (Wobrock et al. 2004). Morphologische Grundlage der Vulnerabilität ist vermutlich Folge einer Hirnentwicklungsstörung, die funktionell mit einer reduzierten Informationsverarbeitungskapazität einhergeht. Zur Erfassung der volumetrischen Veränderungen bei schizophrenen Patienten im Vergleich zu Kontrollpersonen werden derzeit insbesondere computergestützte BildanalyseVerfahren mit verbesserten Segmentierungsalgorithmen in der strukturellen Magnetresonanztomographie eingesetzt (Wobrock et al. 2004). Es konnten mit dem Krankheitsverlauf progredient fortschreitende Volumenverluste der grauen Substanz nachgewiesen werden (DeLisi et al. 1997), so dass neben einer gestörten Hirnentwicklung auch eine neurodegenerative Komponente diskutiert wird. 


\subsubsection{Andere Risikofaktoren}

Geringe körperliche Auffälligkeiten (minor physical abnormalities) wie veränderte Schädelformen und andere kraniofaziale Abweichungen wurden mit einem erhöhten Risiko einer Hirnentwicklungsstörung in Verbindung gebracht. Dazu zählen pränatale Virusinfektionen, Schwangerschaftskomplikationen, Ernährungsdefizite und VitaminD-Mangel. Beim Velokardiofazialen Syndrom findet sich in ausgeprägter Form eine genetisch bedingte Fehlentwicklung. Es handelt sich um eine Mikrodeletion auf dem langen Arm des Chromosoms 22. Diese geht mit einem deutlich erhöhten Risiko für die Entwicklung einer Schizophrenie oder einer bipolaren Psychose einher (Gothelf et al. 1999). Dermatoglyphische Veränderungen, d.h. Abnormitäten der Finger und Hände wie die angedeutete 4-Finger-Falte, Größe, Form und Anzahl der Fingerglieder sind bei Patienten mit Schizophrenie gehäuft zu finden (Fananas et al. 1990). Dies wird unter anderem darauf zurückgeführt, dass die Ausformung der Gliedmaßen genau wie die Hirnentwicklung wesentlich im zweiten Trimester der Schwangerschaft stattfindet.

Der saisonale Faktor der Zeitpunkt der Geburt wurde als ein häufig replizierter Risikofaktor angesehen. So wurde eine Häufung der Schizophrenie in der Bevölkerung der nördlichen Hemisphäre beobachtet. In den späten Wintermonaten und frühen Frühjahrsmonaten Geborene erkrankten häufiger an einer Schizophrenie. Parallel hierzu ergab sich ein reduziertes Risiko für Menschen, die in den späten Sommer-Herbst-Monaten geboren wurden (Torrey et al. 1997). Eine einheitliche Erklärung dieser saisonalen Effekte konnte nicht gegeben werden, vermutet wurden gehäufte perinatale Virusinfekte in der kalten Jahreszeit genauso wie z.B. veränderte Ernährungsgewohnheiten im Vergleich zu den Sommermonaten. Für die südliche Hemisphäre konnte ein solcher saisonaler Zusammenhang nicht hergestellt werden. Auch dem Geburtsort wurde eine Bedeutung als Risikofaktor für die Entwicklung einer Schizophrenie beigemessen. Das relative Risiko für die Entwicklung einer Schizophrenie war bei Geburt in einer städtischen Umgebung um das 2,4-fache gegenüber der Geburt in einer ländlichen Region erhöht. Dabei bestanden in einigen Untersuchungen auch Anhaltspunkte dafür, dass das Risiko mit der Anzahl der Jahre ansteigt, die in der Kindheit in einer (Groß)-Stadt verbracht werden (Pedersen und 
Mortensen 2001). Bei diesem Risikofaktor sind prä- und perinatale Effekte schwer von Einflüssen in der Kindheitsentwicklung zu trennen.

Für Schwangerschafts- und Geburtskomplikationen konnte ein moderater, aber signifikanter Einfluss auf die Entwicklung einer Schizophrenie nachgewiesen werden (Geddes et al. 1999). Dabei handelt es sich vermutlich um einen komplexen Zusammenhang, der eine genaue Auftrennung nach pathophysiologischen Gegebenheiten wie Hypoxie/Ischämie, Infektionen, Mangelernährung und andere nicht zulässt. Mit einem höheren Risiko einer Schizophrenie assoziiert waren z.B. ein niedriges Geburtsgewicht (weniger als 2500g), Frühgeburt, Präeklampsie bei der Mutter, verlängerte Geburtsperiode, Asphyxie und Hypoxie, Blutungen in der Schwangerschaft, Rhesusunverträglichkeit, fetaler Stress und höheres Alter der Mutter.

Passend zur Hypothese der Hirnentwicklungsstörung scheinen prä- und perinatale Komplikationen mit einem ungünstigeren Verlauf der Schizophrenie verknüpft zu sein. Schwangerschafts- und Geburtskomplikationen erhöhen generell das Risiko für eine spätere kognitive Beeinträchtigung. Aus Tierversuchen wurde abgeleitet, dass insbesondere chronisch hypoxische Zustände eine Veränderung der Hirnreifung (Synapsenbildung im glutamatergen System) nach sich ziehen können. Die Vulnerabilität für eine Schädigung durch chronische Hypoxie könnte wiederum genetisch beeinflusst sein (Cannon 1997).

Als weiterer Einflussfaktor gelten pränatale Virusinfektionen. So ergab sich eine Assoziation zwischen Influenza-Epidemien bzw. Exposition und einem erhöhten Risiko für die Entwicklung einer Schizophrenie. Dieser nur in einigen epidemiologischen Studien gefundene Effekt konnte vor allem bei weiblichen Schizophreniekranken belegt werden (McGrath und Murray 2003). Dazu passend konnten experimentell in Tierversuchen Veränderungen der Hirnentwicklung durch die pränatale Infektion mit dem Influenzavirus nachgewiesen werden (Fatemi et al. 1999).

Insgesamt ist jedoch die Wertigkeit von pränatalen Infektionen für die spätere Entwicklung einer Schizophrenie unklar, vermutlich ist von einer geringen unspezifischen Risikoerhöhung für verschiedene ZNS-Erkrankungen auszugehen. 
Tabelle 1: $\quad$ Risikofaktoren der Schizophrenie (Falkai 2003,17)

Faktoren

Das Risiko, an einer Schizophrenie zu erkranken, ist im Vergleich zur NormalBevölkerung erhöht um:

Verwandte 1. Grades mit

einer Schizophrenie

ca. $50 \%$

Schwangerschafts- und

Geburtskomplikationen

ca. $1-2 \%$

Geburtsort

ca. $1-2 \%$

Virusinfektion in der

Schwangerschaft

ca. $1-2 \%$

Drogenmissbrauch

ca. $1-2 \%$

\subsection{Hirnvolumetrische Messungen bei Schizophrenie}

Durch die Entwicklung der Magnetresonanztomographie in den 80er Jahren konnten nichtinvasiv detaillierte morphologische Studien in vivo durchgeführt werden. Ein großer Vorteil der Methode liegt in der Segmentierung des Gehirns, also der Abgrenzung und Quantifizierung von grauer und weißer Substanz sowie von Liquorräumen (Schmitt et al. 2001). Eine detaillierte Aufteilung in kortikale und subkortikale Regionen zeigt, dass die Volumenreduktion nicht einheitlich über das Gehirn verteilt ist, sondern auf distinkte Areale beschränkt bleibt. Im frontalen Kortex zeigen 70\% aller Untersuchungen eine Volumenabnahme, im Thalamus finden 63\% und im Zerebellum 60\% aller Studien eine Größenminderung. Damit haben aus morphologischer Sicht diese Strukturen die größte Bedeutung für die Pathophysiologie der Schizophrenie und für das Auftreten klinischer Symptome (Schmitt et al. 2001).

In den meisten Studien konnte eine Ventrikelerweiterung mit Schwerpunkt im Bereich der Temporalhörner und der Seitenventrikel gefunden werden. Weiterhin wurde ein Verlust zerebraler Asymmetrien wie z.B. des Planum temporale und veränderte 
Gyrierung frontal beobachtet (Vogeley et al. 2001). Auch Falkai et al. (1995) konnten eine Erweiterung des Ventrikelsystems bei ca. 30-40\% der schizophrenen Patienten in einer Vielzahl von postmortalen und bildgebenden Studien feststellen.

Offen bleibt die Frage nach der Ursache der Volumenreduktion. Segmentierungsdaten lassen nur den Schluss zu, dass eine Reduktion der grauen Substanz die Hauptursache der Volumenreduktion ist. Den Defiziten der grauen Substanz können sowohl strukturelle Veränderungen der Neurone als auch der kortikokortikalen und subkortikokortikalen Verbindungen zugrunde liegen. Ursächlich können eine verminderte Anzahl oder Größe mit weniger Zellausläufern bzw. mangelnder Myelinisierung sein (Schmitt et al. 2001).

Außer den lokalisierten Hirnveränderungen finden sich allgemeine Hirnveränderungen, die aber in Bezug auf eine differenzierte Topologie der Schizophrenie wenig ergiebig sind. Darunter sind Berichte über Volumen-, Hirnlängen- und Hirngewichtsveränderungen und Veränderungen der neuronalen Dichte (Benes et al. 1991).

\subsection{Dorsolateraler präfrontaler Kortex - Morphologie und Funktion}

Der präfrontale Kortex (PFC) ist die größte funktionelle Region des menschlichen Gehirns, er umfasst ca. 29\% des gesamten Kortex und ist damit vergleichsweise doppelt so groß wie bei Schimpansen (Andreasen et al. 1992).

In seiner Funktion als massiver Assoziationskortex unterhält er afferente und efferente Verbindungen $\mathrm{zu}$ allen anderen neokortikalen Regionen, sowie zum limbischen System und zu den Basalganglien. Der PFC ist der am meisten komplexe und der höchst entwickelte Anteil des menschlichen Neokortex (Andreasen et al. 1992). Der Thalamus dient als die wichtigste Schaltstelle, der die Afferenzen moduliert, wobei insbesondere zu den dorsomedialen Thalamuskernen ein enger Zusammenhang besteht, so dass diese Verbindung den PFC definiert. Die morphologischen Grenzen des PFC sind der Sulcus arcuatus, der untere Teil des Sulcus praecentralis und der vordere Gyrus cinguli. Der Kortex besteht in dieser Region aus sechs Schichten mit Pyramidenzellen (Andreasen und Black 1993). 
Die optimale neurobiologische Funktion mit kompletter Myelinisierung und Reifung wird beim gesunden Menschen nicht vor der zweiten/dritten Lebensdekade erreicht (Bachneff 1991). Die funktionelle Bedeutung für den Menschen liegt in der Generierung und Modulation spezifischer menschlicher Denkfunktionen, wie sprachlicher und emotionaler Ausdrucksfähigkeit, Planung neuer Ideen sowie Mediation sozialer Interaktionen.

Den präfrontalen Kortex kann man in einen orbitofrontalen, medialen und lateralen Anteil gliedern. Der laterale präfrontale Kortex wird wiederum in dorsolaterale und ventrolaterale Bereiche unterteilt. Brodmann (1909) lokalisiert den dorsolateralen präfrontalen Kortex (DLPFC) auf dem Rindenareal 9/46. Zur Lage der Areale siehe Abbildung 1.


Abbildung 1: Schematische Darstellung von Subregionen des Stirnhirns (Förstl 2004, 22)

Dem DLPFC fällt eine wichtige Rolle in der zeitlichen Organisation von Informationen und dem Zeiterleben zu (Berman et al. 1988). Goldman-Rakic (1987) nimmt an, dass der DLPFC notwendig ist, um internalisierte Repräsentanzen von Situationen oder Stimuli zu bilden, das heißt, die Möglichkeit herzustellen, auf Situationen stärker auf 
der Basis gespeicherter Informationen als auf der Basis unmittelbarer Reizstimulation hin zu antworten.

Weiterhin ist Goldman-Rakic der Ansicht, dass ohne die Regulation des DLPFC die Antwort eines Individuums eine rein stimulusgebundene Reaktion bleibt.

Nach Andreasen und Black (1993) führen Läsionen im DLPFC zu Apathie, Hypokinese und zu Störungen der kognitiven Leistungsfähigkeit.

In einer volumetrischen Untersuchung zeigten die dem heteromodalen Assoziationskortex zugerechneten Regionen superiorer temporaler Gyrus, inferiorer Parietallappen und DLPFC zusammengenommen eine Volumenminderung bei schizophren Erkrankten im Vergleich zu Gesunden. Diese Verminderung konnte für Bipolare Patienten nicht gezeigt werden (Schlaepfer et al. 1994).

Laut Fuster (1997) ist der DLPFC eine für das Arbeitsgedächtnis kritische Hirnstruktur, die mit dem temporolimbischen, parietalen und cingulären Kortex in enger Verbindung steht. Zu den Aufgaben des Arbeitsgedächtnisses gehören die vorübergehende Speicherung (z.B. Sätze inhaltlich zu verstehen), die Lösung komplexer Aufgaben, das problemlösende Denken und der Erwerb neuen Wissens. Ferner gehören das Verständnis der gegenwärtig umgebenden Umwelt und die mentale Repräsentation dieser dazu.

Nach Goldman-Rakic und Selemon (1997) zählen zu den wesentlichen Merkmalen des Arbeitsgedächtnisses die aktive Verarbeitung von verbalem oder visuellräumlichem Material, die Manipulation und Wiederholung neuer Informationen, sowie das Sicherstellen der Kontinuität zwischen vergangener Erfahrung und gegenwärtigen Prozessen. Einbußen in Arbeitsgedächtnisaufgaben gelten seit langem als zentrale neuropsychologische Fehlfunktion bei der Schizophrenie. Insbesondere wenn an Schizophrenie Erkrankte Aufgaben lösen, die das Arbeitsgedächtnis oder die exekutive Handlungskontrolle beanspruchen, konnte eine gestörte Aktivierung des DLPFC in bildgebenden Studien repliziert werden (Silver et al. 2003). 


\section{Material und Methoden}

\subsection{Fragestellung der Arbeit}

In der vorliegenden Dissertation wurde untersucht, ob eine Verminderung der Volumina des DLPFC bei Schizophrenie im Vergleich zu gesunden Kontrollen vorliegt.

Weiterhin stellte sich die Frage, ob ein eventuell gefundener Unterschied (Reduktion) diagnosespezifisch ist. Deswegen wurde im Vergleich bei einer Gruppe von Bipolaren Patienten und Zwangspatienten ebenfalls das Volumen des DLPFC bestimmt.

Abschließend erfolgte eine Korrelation der volumetrischen Befunde mit soziodemografischen und klinischen Variablen der untersuchten Stichproben, um mögliche Zusammenhänge aufzuzeigen.

\subsection{Beschreibung der verwendeten psychopathologischen Skalen}

Die Positive and Negative Syndrome Scale (PANSS) gehört seit ihrer Entwicklung von Kay et al. 1987 zu den maßgebenden Skalen zur Erfassung der Psychopathologie in der Schizophrenieforschung (Bryson et al. 1999). Die Skala beurteilt die positiven und negativen Dimensionen der schizophrenen Erkrankung. Unter Positivsymptomatik werden Wahn, Halluzination (beispielsweise akustische, optische und den eigenen Körper betreffende Halluzinationen) und IchErlebnisstörungen (Gedankeneingebung, -ausbreitung, -entzug) gezählt. Zur Negativsymptomatik zählt man Antriebsmangel, Affektstörungen, Alogie, Anhedonie, Mangel an sozialen Kontakten sowie Konzentrationsstörungen. Diese Symptome werden in einem 30-40 minütigen formalisierten psychiatrischen Interview anhand einer siebenstufigen Skala von 1 (nicht vorhanden) bis 7 (extrem ausgeprägt) bewertet. Jedes Symptom ist einer von drei Skalen zugeordnet, sieben der Positivskala, weitere sieben der Negativskala und die restlichen 16 der Globalskala. Diese Beurteilung bezieht sich auf die Befindlichkeit der Person während der vorangegangenen sieben Tage. Es können ebenfalls Informationen durch das 
Krankenhauspersonal oder Angehörige mit in die Bewertung einfließen. Aus der PANSS ergibt sich ein Summenscore von 30 bis 120 (Kay et al. 1987).

Die Global Assessment of Functioning Skala (GAF) dient einer globalen Beurteilung des Funktionsniveaus in sozialen und beruflichen Bereichen. Dabei können Werte von 0 bis 100 Punkte erzielt werden. Die Abstufung der Skala erfolgt jeweils in 10er Bereichen. Von einer nahezu unbeeinträchtigten Leistungsfähigkeit ist beispielsweise bei einem Wert bis zu 80 Punkten auszugehen. Diese Skala wurde dem Diagnostischen und Statistischen Manual IV (DSM-IV; Saß et al. 1996) entnommen.

Die Skala Clinical Global Impressions (CGI) (Guy 1976/2000) ist eine Möglichkeit, den klinischen Zustand bzw. den Schweregrad der Erkrankung des Patienten mit Hilfe von 8 Stufen zu beurteilen. Dabei reicht die Skala von 0 als nicht beurteilbar, über 1 überhaupt nicht krank bis hin zu 7 extrem schwer krank.

Bei den Bipolaren Patienten wurde die Anzahl der depressiven und manischen Episoden vermerkt. Außerdem wurde jeweils der Score in der Montgomery Asberg Depression Rating Scale (MADRS) und in der Young Mania Rating Scale (YMRS) Score erhoben.

Der MADRS gibt einen Schweregrad für die Depression an und ist unterteilt in zehn Bereiche wie Traurigkeit, Innere Anspannung, Appetit oder Konzentrationsschwierigkeiten. In jedem Teilbereich gibt es eine Punktzahl von 0 (keine Probleme) bis 6 (sehr starke Probleme), daraus ergibt sich ein Summenscore von maximal 60 Punkten (Montgomery und Asberg 1979).

Die YMRS gibt einen Score an für den Schweregrad der Manie. Die YMRS besteht aus 11 Fragen (Young et al. 1978). Diese beziehen sich auf die letzten 48 Stunden. In den Fragen werden manische Symptome wie vermindertes Schlafbedürfnis, gesteigerte Energie, gehobene Stimmung und Antrieb, Reizbarkeit und aggressives Verhalten, veränderte Denkinhalte und Anderes abgefragt. Je stärker ein Symptom ausgeprägt ist, desto mehr Punkte werden pro Frage verliehen. Bei den Fragen 6, 8 und 9 können bis zu 8 Punkte vergeben werden, bei allen anderen Fragen bis zu 4 Punkte mit Abstufungen von einem halben Punkt. Nicht nur die Angaben des 
Patienten, sondern auch der klinische Eindruck und Angaben von Angehörigen können in die Bewertung einfließen. Insgesamt können 60 Punkte erreicht werden (Young et al. 1978).

\subsection{Klinische und soziodemographische Daten der Patientengruppen}

Die MRT-Aufnahmen stammten alle von Probanden aus dem Patientenkollektiv der Klinik für Psychiatrie und Psychotherapie des Universitätsklinikums des Saarlandes. Die Probanden bzw. Patienten wurden vor Beginn der Studie ausführlich aufgeklärt und gaben schriftlich Ihre Einwilligung zur Teilnahme (Informed Consent). Für die Studie lag ein positives Votum der zuständigen Ethik-Kommission (Ärztekammer des Saarlandes) vor.

\subsubsection{Deskriptive Statistik der Gruppe 'schizophrene Patienten'}

Die ersterkrankten Schizophrenen stellten mit 61 Personen die zweitgrößte Patientengruppe dar. Die größte untersuchte Gruppe überhaupt stellten die gesunden Kontrollen mit 69 Personen dar. Berücksichtigt wurden die Faktoren 'Alter', 'Größe', 'Gewicht' und 'Bildung'. Bei dem Bildungsstand waren sowohl die eigene Schulbildung als auch die Schulbildung der Eltern relevant. Weiterhin von Bedeutung waren das Gesamthirnvolumen, die Prodromdauer, die Ausprägung der Psychopathologie (PANSS), der Funktionseinschränkung (GAF) und des Schweregrades der Erkrankung (CGI). Alle Parameter wurden zum Zeitpunkt der stationären Aufnahme ermittelt. Für alle Faktoren wurden Mittelwerte und Standardabweichungen bestimmt. 
Tabelle 2

\begin{tabular}{|c|c|c|c|c|}
\hline \multicolumn{5}{|c|}{$\begin{array}{l}\text { Soziodemografische und klinische Variablen der ersterkrankten } \\
\qquad \text { Schizophrenen (Samplegröße } n=61 \text { ) }\end{array}$} \\
\hline & Minimum & Maximum & Mittelwert & Standardabweichung \\
\hline Alter (in Jahren) & 18 & 46 & 28,57 & 7,639 \\
\hline Körpergröße (cm) & 155 & 195 & 175,79 & 9,651 \\
\hline $\begin{array}{l}\text { Körpergewicht } \\
(\mathrm{kg})\end{array}$ & 55,0 & 178,0 & 78,603 & 20,9483 \\
\hline $\begin{array}{l}\text { Psychosedauer } \\
\text { (Wochen) }\end{array}$ & 1 & 268 & 42,70 & 55,449 \\
\hline $\begin{array}{l}\text { Prodromdauer } \\
\text { (Wochen) }\end{array}$ & 2 & 720 & 168,84 & 160,050 \\
\hline $\begin{array}{l}\text { Alter bei } \\
\text { Ersterkrankung } \\
\text { (Jahre) }\end{array}$ & 17 & 45 & 27,39 & 7,815 \\
\hline $\begin{array}{l}\text { Maximale Anzahl } \\
\text { der Schuljahre } \\
\text { inklusive Studium } \\
\text { insgesamt }\end{array}$ & 8 & 20 & 12,62 & 2,951 \\
\hline $\begin{array}{l}\text { Anzahl der } \\
\text { Schuljahre ohne } \\
\text { spätere } \\
\text { Ausbildung }\end{array}$ & 8 & 13 & 10,49 & 1,757 \\
\hline $\begin{array}{l}\text { Maximale Anzahl } \\
\text { der Schuljahre } \\
\text { inklusive Studium } \\
\text { des Vaters }\end{array}$ & 8 & 20 & 12,52 & 2,837 \\
\hline $\begin{array}{l}\text { Anzahl der } \\
\text { Schuljahre ohne } \\
\text { spätere } \\
\text { Ausbildung des } \\
\text { Vaters }\end{array}$ & 8 & 13 & 9,55 & 1,899 \\
\hline
\end{tabular}


Tabelle 3

\begin{tabular}{|c|c|c|c|c|}
\hline \multicolumn{5}{|c|}{$\begin{array}{l}\text { Soziodemografische und klinische Variablen der ersterkrankten } \\
\text { Schizophrenen (Samplegröße n=61) (Fortsetzung) }\end{array}$} \\
\hline & Minimum & Maximum & Mittelwert & Standardabweichung \\
\hline $\begin{array}{l}\text { Maximale Anzahl } \\
\text { der Schuljahre } \\
\text { inklusive Studium } \\
\text { der Mutter }\end{array}$ & 8 & 18 & 10,63 & 2,571 \\
\hline $\begin{array}{l}\text { Anzahl der } \\
\text { Schuljahre ohne } \\
\text { spätere } \\
\text { Ausbildung der } \\
\text { Mutter }\end{array}$ & 8 & 13 & 9,22 & 1,552 \\
\hline $\begin{array}{l}\text { PANSS positiv } \\
\text { Score }\end{array}$ & 8 & 35 & 21,75 & 5,991 \\
\hline $\begin{array}{l}\text { PANSS negativ } \\
\text { Score }\end{array}$ & 8 & 36 & 22,18 & 6,871 \\
\hline $\begin{array}{l}\text { PANSS } \\
\text { allgemeine PP- } \\
\text { Score }\end{array}$ & 25 & 65 & 47,77 & 9,531 \\
\hline $\begin{array}{l}\text { PANSS Gesamt- } \\
\text { Score }\end{array}$ & 43 & 123 & 91,67 & 17,434 \\
\hline $\begin{array}{l}\text { GAF bei } \\
\text { Aufnahme }\end{array}$ & 15 & 65 & 30,10 & 12,348 \\
\hline $\begin{array}{l}\text { CGI bei } \\
\text { Aufnahme }\end{array}$ & 4 & 7 & 5,83 & 0,668 \\
\hline $\begin{array}{l}\text { MADRS-Gesamt- } \\
\text { Score }\end{array}$ & 6 & 38 & 19,30 & 6,641 \\
\hline
\end{tabular}

PANSS=Positive and Negative Syndrom Scale, PP=Psychopathologie, GAF=Global Assessment of Functioning, $\mathrm{CGI}=\mathrm{Clinical}$ Global Impressions, MADRS= Montgomery-Asberg depression rating scale 


\subsubsection{Deskriptive Statistik der Gruppe 'Bipolare Patienten‘}

Zu dieser Gruppe zählten 44 Personen im Alter zwischen 22 und 66 Jahren. Die Ausbildungsdauer lag zwischen 8 und 18 Jahren. Die Ausbildungsdauer der Eltern lag bei 8 bis 18 Jahren für den Vater. Zwischen 8 bis 18 Jahren für die Mutter.

Für die statistische Auswertung wurden Mittelwerte und Standardabweichung für jeden dieser Faktoren berechnet.

Tabelle 4

\begin{tabular}{|l|c|c|c|c|}
\hline \multicolumn{5}{|c|}{ Soziodemografische Variablen der Bipolaren Patienten } \\
(Samplegröße n=44)
\end{tabular}

\subsubsection{Deskriptive Statistik der Gruppe 'Zwangspatienten'}

Zu den Zwangspatienten zählten 30 Personen zwischen 19 und 54 Jahren. Die Ausbildungsdauer lag zwischen 8 und 18 Jahren. Die Ausbildungsdauer der Eltern lag jeweils bei 9 bis 18 Jahren. Bei Erstuntersuchung wurde bei allen Patienten das gesamte Hirnvolumen gemessen.

Für die statistische Auswertung wurden Mittelwerte und Standardabweichung für jeden dieser Faktoren berechnet. 
Tabelle 5

\begin{tabular}{|c|c|c|c|c|}
\hline \multicolumn{5}{|c|}{$\begin{array}{l}\text { Soziodemografische Variablen der Zwangspatienten } \\
\qquad(\text { Samplegröße } n=25)\end{array}$} \\
\hline & Minimum & Maximum & Mittelwert & $\begin{array}{l}\text { Standard- } \\
\text { abweichung }\end{array}$ \\
\hline Alter (in Jahren) & 19 & 54 & 35,30 & 9,483 \\
\hline $\begin{array}{l}\text { Maximale Anzahl der } \\
\text { Schuljahre inklusive } \\
\text { Studium insgesamt }\end{array}$ & 8 & 18 & 12,72 & 2,011 \\
\hline $\begin{array}{l}\text { Maximale Anzahl der } \\
\text { Schuljahre inklusive } \\
\text { Studium des Vaters }\end{array}$ & 9 & 18 & 12,50 & 2,106 \\
\hline $\begin{array}{l}\text { Maximale Anzahl der } \\
\text { Schuljahre inklusive } \\
\text { Studium der Mutter }\end{array}$ & 9 & 18 & 12,50 & 2,106 \\
\hline
\end{tabular}

\subsubsection{Deskriptive Statistik der Gruppe 'gesunde Kontrollen`}

Zu den gesunden Kontrollen zählten 69 Personen zwischen 19 und 66 Jahren. Von einem Großteil der Kontrollen wurden Körpergröße (Person mit geringster Größe 160 cm, größte Person $195 \mathrm{~cm}$ ) und Gewicht (leichteste Person 55 kg, schwerste Person $130 \mathrm{~kg}$ ) mitberücksichtigt. Die Ausbildungsdauer lag zwischen 12 und 19 Jahren. Die Ausbildungsdauer der Eltern lag bei 9 bis 18 Jahren für den Vater und 9 bis 18 Jahren für die Mutter. Es wurden zusätzlich jeweils von der betreffenden Person, deren Vater und deren Mutter, die Schuljahre ohne anschließende Ausbildung berücksichtigt. Für die statistische Auswertung wurden Mittelwerte und Standardabweichung für jeden dieser Faktoren berechnet. 
Tabelle 6

\begin{tabular}{|c|c|c|c|c|}
\hline \multicolumn{5}{|c|}{$\begin{array}{l}\text { Soziodemografische Variablen der gesunde } \\
\qquad \text { (Samplegröße } n=69 \text { ) }\end{array}$} \\
\hline & Minimum & Maximum & Mittelwert & $\begin{array}{l}\text { Standard- } \\
\text { abweichung }\end{array}$ \\
\hline Alter (in Jahren) & 19 & 66 & 33,76 & 9,849 \\
\hline Körpergröße (cm) & 160 & 195 & 177,61 & 8,340 \\
\hline Körpergewicht (kg) & 55,0 & 130,0 & 77,984 & 18,3999 \\
\hline $\begin{array}{l}\text { Maximale Anzahl der } \\
\text { Schuljahre inklusive } \\
\text { Studium insgesamt }\end{array}$ & 12 & 19 & 15,16 & 2,542 \\
\hline $\begin{array}{l}\text { Anzahl der Schuljahre } \\
\text { ohne spätere Ausbildung }\end{array}$ & 9 & 13 & 11,79 & 1,695 \\
\hline $\begin{array}{l}\text { Maximale Anzahl der } \\
\text { Schuljahre inklusive } \\
\text { Studium des Vaters }\end{array}$ & 9 & 18 & 14,27 & 2,959 \\
\hline $\begin{array}{l}\text { Anzahl der Schuljahre } \\
\text { ohne spätere Ausbildung } \\
\text { des Vaters }\end{array}$ & 8 & 13 & 10,50 & 1,871 \\
\hline $\begin{array}{l}\text { Maximale Anzahl der } \\
\text { Schuljahre inklusive } \\
\text { Studium der Mutter }\end{array}$ & 9 & 18 & 12,33 & 2,578 \\
\hline $\begin{array}{l}\text { Anzahl der Schuljahre } \\
\text { ohne spätere Ausbildung } \\
\text { der Mutter }\end{array}$ & 8 & 13 & 9,97 & 1,497 \\
\hline
\end{tabular}

\subsection{MR-technische Parameter und Volumetrie}

Die MR-Untersuchungen wurden an einem 1,5-Tesla-Scanner der Fa. Siemens, Typ Sonata, in der Abteilung für Neuroradiologie des Universitätsklinikums des Saarlandes durchgeführt. Die volumetrischen Auswertungen erfolgten an T1gewichteten Bildern, Sequenz IR/GR als MPRAGE (Magnetization Prepared Rapid Gradient Echo), Typ 3D, Voxel 1x1×1 cmm; Matrix 176 × 256 × 256, mit den Zeiten TR = 1900 ms; TE = 4 ms; TI = 700 ms; Flip-Winkel 15 Grad. 
Die Volumetrie erfolgte mit dem Programm MRICroN, einem Imaging Tool, welches ANALYZE-Formate, DICOM, NEMA, GE, Interfile, ECAT, Picker, Siemens, Elscint, VFF, VoxBo, und Rohbilddaten verwerten (lesen) kann. Mit MRIcroN kann auch in SPM konvertiert werden. Die Visualisierung schließt Standardschnitte, schiefe Schnittebenen und Volumenmarkierungen ein. Mit MRICroN können bestimmte Regionen markiert und die umfahrene Fläche gemessen werden, bei mehreren Schnitten wird durch die Angabe der Anzahl der Voxel das Volumen ermittelt (Ansatz des Region of Interest, ROI). Das Programm wurde von Chris Rorden, University of South Carolina, entwickelt - frei verfügbar unter der Internet-Adresse: http://www.sph.sc.edu/comd/rorden/mricro.html.

Das Gesamtvolumen der grauen Substanz wurde in einem semiautomatisierten Messverfahren erfasst, wobei die Software SPM2 (Statistical Parametric Mapping, Wellcome Department of Cognitive Neurology, London, UK) verwendet wurde. SPM ist eine Software zum Berechnen von fMRT- und PET-Daten, die als MATLABApplikation programmiert ist. Nach dem Preprocessing wurden mit Hilfe der Segment-Funktion von SPM99 (http://www.fil.ion.ucl.ac.uk/spm/) die T1-gewichteten MR-Scans 'graue Substanz', 'weiße Substanz' und 'Liquorräume' segmentiert. Anschließend konnte das Volumen der grauen Substanz anhand der segmentierten Voxelzahl mit MATLAB berechnet werden.

Das gesamte Volumen des präfrontalen Kortex (PFC) wurde mit einer automatisierten Parcellationsprozedur berechnet, welche in der Software zur Berechnung des automaisierten Gyrifizierungsindex der Arbeitsgruppe um Prof. Stephen Lawrie der Universität Edinburgh implementiert ist. Die Volumenberechnungen wurden mit den übersandten MR-Scans im Analyze-Format direkt in Edinburgh von dem Entwickler des Verfahrens, Thomas William Moorhead, vorgenommen (Bonnici et al. 2007). 


\subsection{Definition der anatomischen Grenzen des DLPFC}

Die Ausdehnung des DLPFC wurde auf den Gyrus frontalis superior und den Gyrus frontalis medius bezogen.

Gemessen wurde ausschließlich die graue Substanz, da sich die Messung der weißen Substanz bei den ersten Versuchen nicht reproduzierbar realisieren ließ.

Die Markierung des DLPFC fand in der Koronarebene statt. Die Horizontal- und Sagittalebene dienten der Orientierung und Korrektur.

Als Hintergrund wurde die MRICroN-Programmeinstellung 'actc' gewählt, der Kontrast war mit 1,3984 und 81,1094 bei allen Schichten eingestellt. Mit dieser Einstellung ließ sich die graue Substanz am besten abgrenzen.

\subsubsection{Start- und Endschicht}

Die Markierung des DLPFC erfolgt von dorsal nach rostral. Zur Definition der Startschicht und Endschicht wird der Medianschnitt in der Sagittalebene markiert. Als Orientierung hierfür hilft der Thalamus. Im Medianschnitt wird durch eine Senkrechte am vorderen Knie des Corpus callosum die Startschicht bestimmt. Die Senkrechte markiert die erste Schicht, die nicht mehr zum Corpus callosum zuzuordnen ist.

Die Endschicht wird durch eine Senkrechte im Sulcus superior festgelegt. Die letzte Schicht vor dem Sulcus superior dient zur Markierung der Senkrechten. Da die Region des DLPFC anatomisch nicht eindeutig abgrenzbar ist, wurde diese Grenze gewählt, auch wenn die Ausdehnung des Gyrus frontalis superior und des Gyrus frontalis medius größer war.

\subsubsection{Laterale Grenze}

Lateral wird der DLPFC durch den Sulcus frontalis inferior gegen den Gyrus frontalis inferior abgegrenzt. 


\subsubsection{Mediale Grenze}

Median ist der DLPFC balkennah gegen den Gyrus cinguli abzugrenzen. Grenzgebend ist hier der Sulcus cinguli. Balkenfern nach rostral muss der DLPFC gegen den Gyrus rostralis superior abgegrenzt werden.

Anfangspunkt der Grenzziehung ist jeweils der tiefste Punkt der Sulci.

Für die Festlegung der anatomischen Grenzen fand eine Orientierung an den Studien von Crespo-Facorro et al. (1999) und Falkai et al. (2004) statt (näheres in der Diskussion).

Außerdem wurde mit Hilfe folgender anatomischer Atlanten gearbeitet:

- Co-Planar Stereotaxic Atlas of the human Brain; 3-Dimensional Proportional System: An Approach to Cerebral Imaging; Jean Talairach, Pierre Tournoux, Thieme, Stuttgart 1988

- $\quad$ The Human Brain; Surface, Blood Supply and Three-Dimensional Sectional Anatomy; Henri M Duvernoy; Springer Wien, New York 1999

- $\quad$ Atlas of the Human Brain; Jürgen K Mai, Joseph Assheuer, George Paxinos; Academic Press, San Diego 1997 


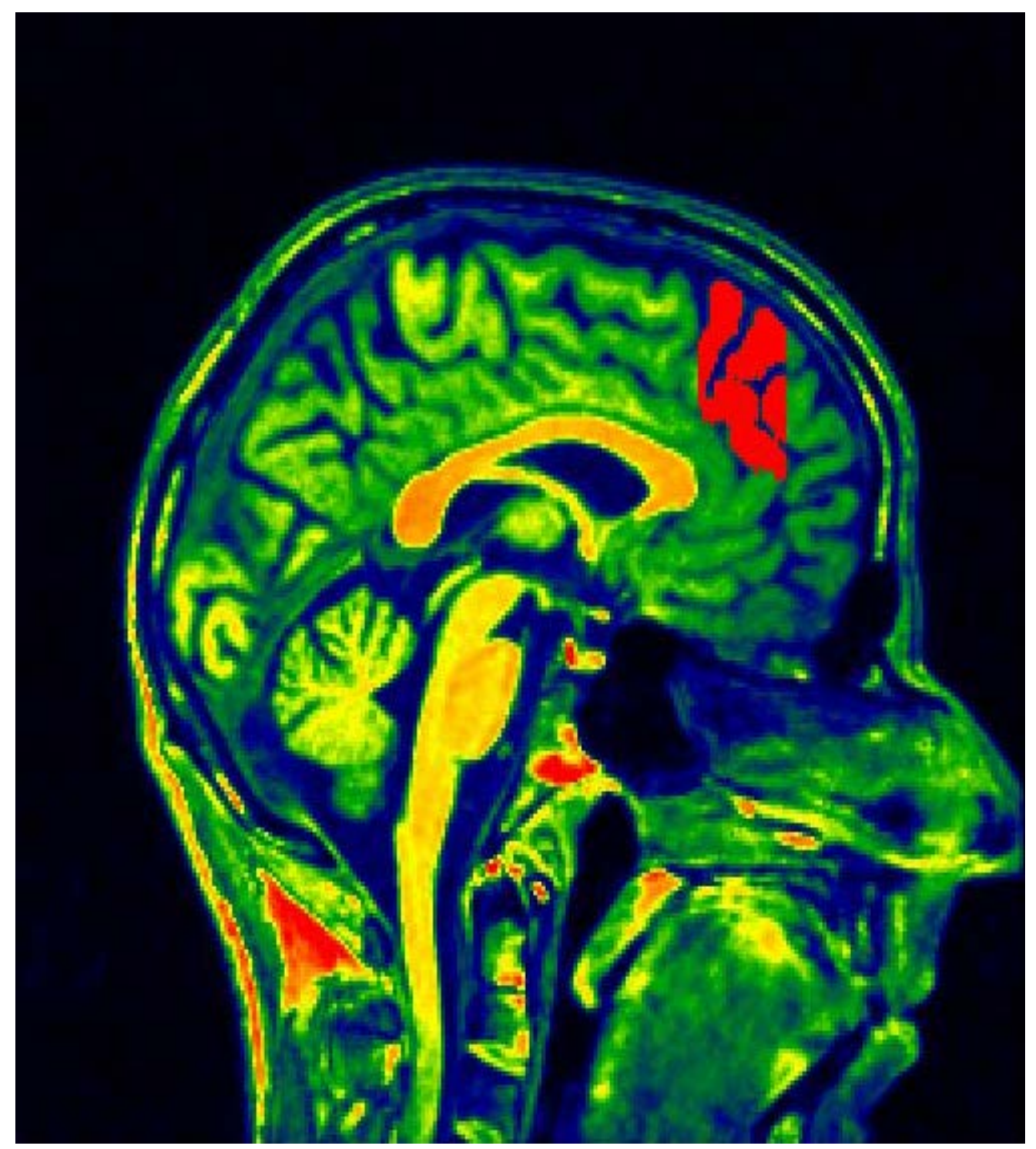

Abbildung 2

Markierung des DLPFC in der Medianebene, T1-gewichtetes MR-Schnittbild 


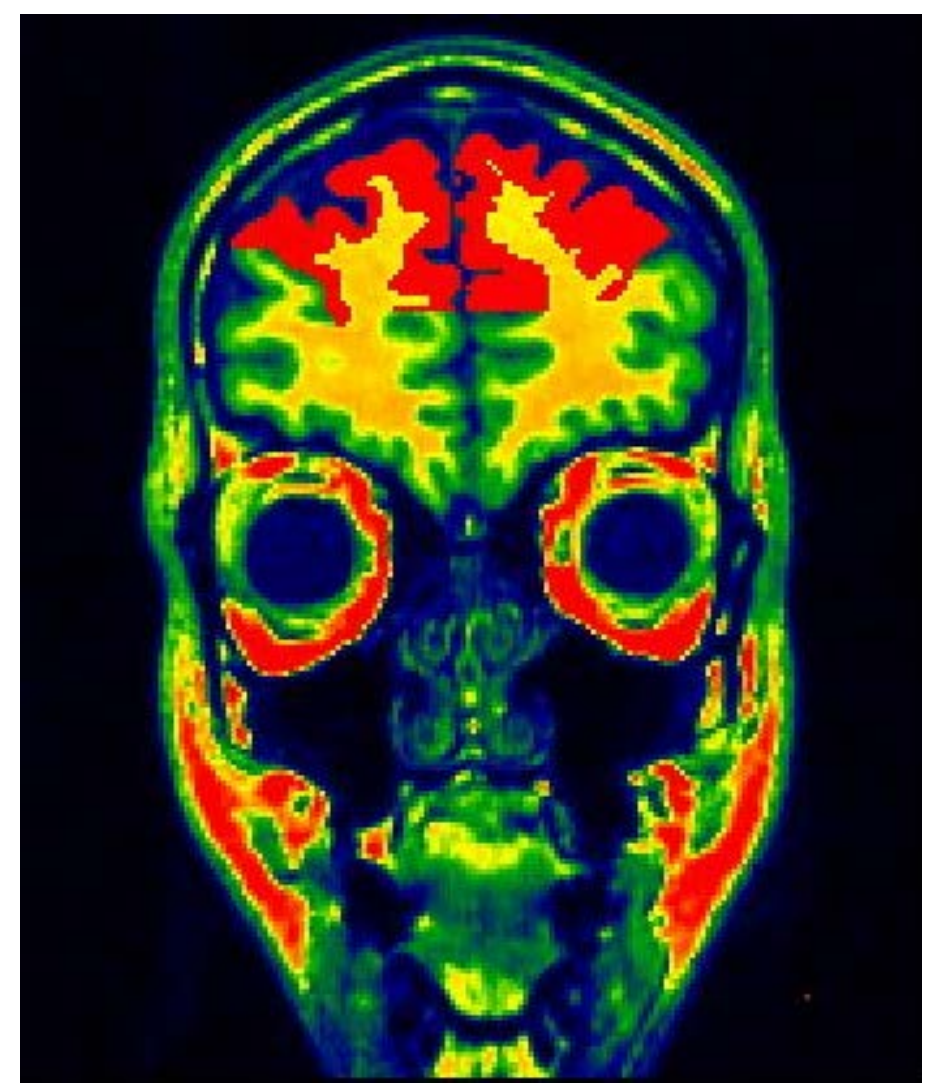

Abbildung 3

Markierung des DLPFC in der Koronarebene, T1-gewichtetes MR-Schnittbild

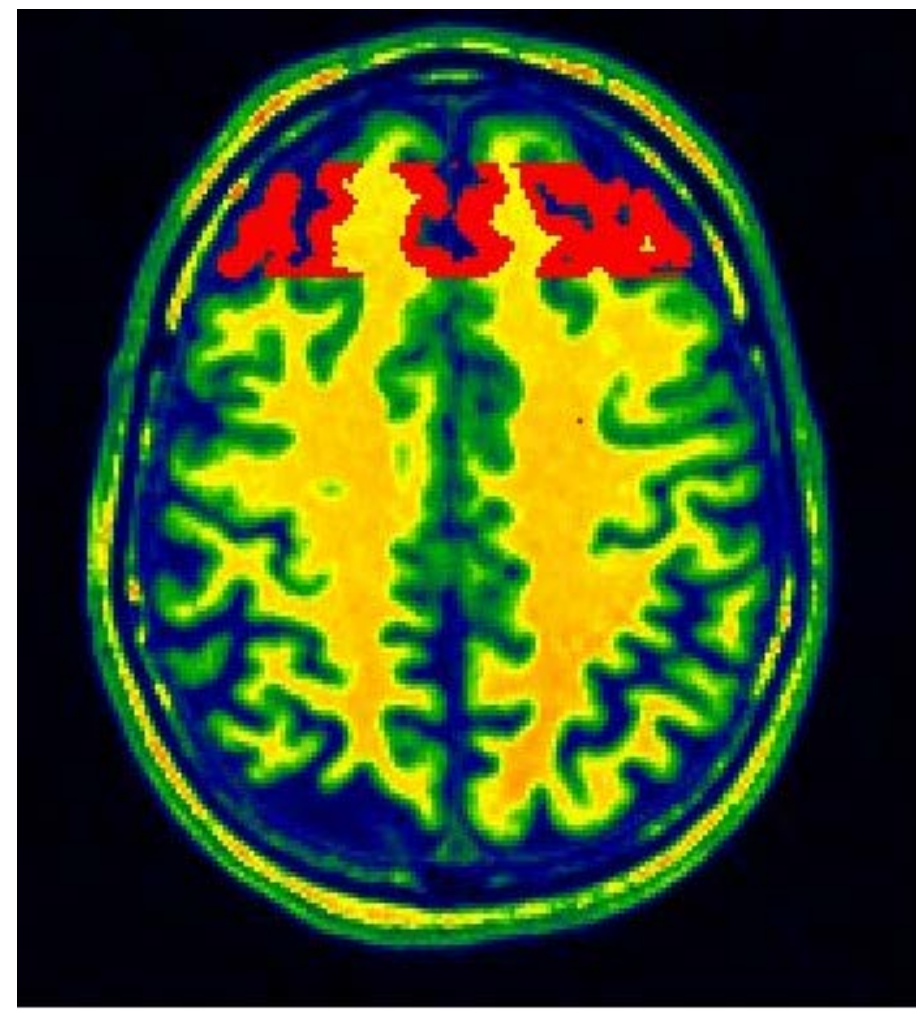

Abbildung 4

Markierung des DLPFC in der Horizontalebene, T1-gewichtetes MR-Schnittbild 


\subsection{Statistische Methode}

Zur Berechnung statistischer Unterschiede zwischen den Gruppen bei normalverteilten Variablen wurde die Methode der Varianzanalyse verwendet.

Als Varianzanalyse bezeichnet man eine Gruppe datenanalytischer und mustererkennender statistischer Verfahren, die zahlreiche unterschiedliche Anwendungen zulassen. Ihnen gemeinsam ist, dass sie Varianzen und Prüfgrößen berechnen, um Aufschlüsse über die hinter den Daten steckenden Gesetzmäßigkeiten zu erlangen. Die Varianz einer oder mehrerer Zielvariable(n) (oder auch 'abhängige Variablen' genannt) wird dabei durch den Einfluss einer oder mehrerer Einflussvariablen (Faktoren, wie z.B. die Diagnose) erklärt. Je nachdem, ob eine oder mehrere Zielvariablen vorliegen, unterscheidet man zwei Formen der Varianzanalyse.

1. Die univariate Varianzanalyse, nach der englischen Bezeichnung analysis of variance auch als ANOVA bezeichnet.

2. die multivariate Varianzanalyse, nach der englischen Bezeichnung multivariate analysis of variance auch als MANOVA bezeichnet.

Je nachdem, ob ein oder mehrere Faktoren vorliegen, unterscheidet man zwischen einfaktorieller (einfacher) und mehrfaktorieller Varianzanalyse. Werden zusätzliche andere Faktoren als mögliche intervenierende Variablen (Einflussvariablen oder Kovariaten) berücksichtigt, so wird eine uni- oder multifaktorielle Kovarianzanalyse (ANCOVA oder MANCOVA) durchgeführt.

Mit Hilfe der Bonferroni-Methode oder Bonferroni-Korrektur wird die AlphafehlerKumulierung bei multiplen Paarvergleichen neutralisiert, also die Fehlerwahrscheinlichkeit für multiples Testen korrigiert.

Die statistische Auswertung der erhobenen Daten in Bezug auf die Fragestellungen der Arbeit wurde wie folgt vorgenommen: 
Als abhängige Variablen (Zielvariablen) wurden die absoluten Volumenwerte für den DLPFC sowie die relativen Volumenwerte für den DLPFC, d.h. der Quotient aus absolutem DLPFC und gesamten Volumen der grauen bzw. weißen Substanz (DLPFC/GS bzw. WS), beidseits verwendet. Weiterhin wurden als weitere relative Volumina das relative Volumen des DLPFC bezogen auf das Gesamtvolumen des präfrontalen Kortex beidseits verwendet (DLPFC/PFC). Die Bestimmung der relativen Volumina ist deshalb von Bedeutung um regionale Veränderungen des DLPFC als Zielregion unabhängig vom Gesamthirnvolumen bzw. der über übergeordneten Region nachweisen zu können.

Die Zielvariablen wurden folgendermaßen ermittelt:

- Die mit dem Programm MRICroN angezeigten Voxelzahlen des Volume of Interest (VOI) (hier: DLPFC) entsprachen den Volumina in cmm (1 Voxel entspricht 1 Kubikmillimeter). Diese absoluten Volumina in $\mathrm{cmm}$ wurden in eine Tabelle übertragen.

- Unter Zuhilfenahme des Gesamthirnvolumens wurde das jeweilige relative DLPFC-Volumen als Quotient aus absolutem DLPFC-Volumen und Gesamthirnvolumen berechnet.

Bei der diagnoseübergreifenden weiteren statistischen Auswertung wurden als abhängige Variablen die Volumina (absolute DLPFC-Volumina links und rechts in $\mathrm{cmm}$, relative DLPFC-Volumina links und rechts in \% des Gesamthirnvolumens und der DLPFC-Asymmetriekoeffizient nach Galaburda) und als unabhängige Variablen die Diagnosegruppen betrachtet.

Der DLPFC-Asymmetriekoeffizient AI wurde wie folgt berechnet:

- $\mathrm{Al}=(2 *(\mathrm{Vr} .-\mathrm{VI}) /.(\mathrm{Vr}$. $+\mathrm{VI}$. $))$, wobei $\mathrm{V}=$ Volumen; $\mathrm{r}=$ rechts und $\mathrm{I}=$ links.

Als intervenierende Variablen wurden untersucht:

- Geschlecht

- Händigkeit

- Alter 
- Bildungsdauer

- für die Bipolare Störung: Krankheitsdauer, Ersterkrankungsalter, Anzahl depressiver u. manischer Episoden, MADRS, YMRS, Psychotische Symptome, Neuroleptika, Lithium, andere Stimmungsaufheller

- für die Schizophren Ersterkrankten: Psychosedauer (Wochen), ProdromDauer, PANSS (positiv, negativ, allgemein, total), CGI, GAF, CPZ (Tagesdosis u. kumulative Gesamtdosis)

Bei der Hauptauswertung wurde anhand der gefundenen relevanten Einflussfaktoren auf das Gesamthirnvolumen (graue Substanz) eine multivariate Kovarianzanalyse vorgenommen mit den abhängigen Variablen absolutes DLPFC-Volumen, relatives DLPFC-Volumen und den Faktoren Diagnose, Geschlecht sowie den Kovariaten Alter, Bildungsdauer (MANCOVA).

Anschließend erfolgten noch Subgruppenanalysen mit jeweils Diagnosegruppen (Schizophrene vs. Kontrollen, Bipolar vs. Kontrollen, OCD vs. Kontrollen) mit Korrektur der p-Werte nach Bonferroni.

Das statistische Signifikanzniveau wurde für die Analysen auf alpha $<0,05$ festgelegt.

Für die statistische Auswertung wurde das Programm SPSS für PC, Version 16.0 verwendet. 


\section{Ergebnisse}

\section{1 Überprüfung auf Reliabilität}

Die Reliabilität ist ein Maß für die Güte der Methode, die zur Messung einer bestimmten Variablen eingesetzt werden.

Zur Überprüfung der Güte der Messungen wurde zunächst die Intra-Rater- oder Retest-Reliabilität bestimmt, d.h. an einem bestimmten Objekt (hier: DLPFCVolumen) wird zweimal dieselbe Messung vorgenommen und die Messwerte miteinander verglichen. Die Retest Reliabilität wurde mittels der Intraclasscorrelation (ICC) berechnet und war mit ICC $>0,99$ bei $n=10$ sehr hoch.

Tabelle 7

\begin{tabular}{|l|l|c|c|}
\hline \multicolumn{4}{|c|}{ Berechnung der Intrarater-Reliabilität } \\
\hline & & Pearson-Korrektur & Intraklassen-Korr. (ICC) \\
\hline $\begin{array}{l}\text { DLPFC total } \\
\text { (GS) }\end{array}$ & $\mathrm{p}$ & 0,998 & 0,998 \\
& $\mathrm{p}$ & $<0,0005$ & \\
\hline $\begin{array}{l}\text { DLPFC links } \\
\text { (GS) }\end{array}$ & $\mathrm{p}$ & 10 & 0,996 \\
& $\mathrm{p}$ & 0,997 & \\
\hline DLPFC & $\mathrm{n}$ & 10 & 0,991 \\
rechts (GS) & $\mathrm{p}$ & 0,0005 & \\
& $\mathrm{n}$ & 10 & \\
\hline
\end{tabular}

Zusätzlich wurden bei Probanden die Messungen von zwei unabhängigen Untersuchern vorgenommen und die Ergebnisse der DLPFC-Volumina miteinander 
verglichen. Auch die Interrater Reliabilität wurde mittels ICC berechnet und bei $n=10$ betrug die ICC>0,960.

Tabelle 8

\begin{tabular}{|l|l|c|c|}
\hline \multicolumn{4}{|c|}{ Berechnung der Interrater-Reliabilität } \\
\hline & & $\begin{array}{c}\text { Pearson- } \\
\text { Korrektur. }\end{array}$ & Intraklassen-Korr. (ICC) \\
\hline $\begin{array}{l}\text { DLPFC total } \\
\text { (GS) }\end{array}$ & r & 0,973 & 0,960 \\
& $\mathrm{p}$ & $<0,0005$ & \\
\hline DLPFC links & $\mathrm{r}$ & 10 & 0,962 \\
(GS) & $\mathrm{p}$ & 0,974 & \\
& $\mathrm{n}$ & $<0,0005$ & \\
\hline DLPFC & $\mathrm{r}$ & 10 & 0,956 \\
rechts (GS) & $\mathrm{p}$ & 0,970 & \\
& $\mathrm{~N}$ & $<0,0005$ & \\
\hline
\end{tabular}

DLPFC =dorsolateraler präfrontaler Kortex, GS=graue Substanz

\subsection{Soziodemografische und klinische Variablen der untersuchten MR- Stichproben im Vergleich}

Von den in die Gesamtstudie eingeschlossenen Probanden konnten aus verschiedenen Gründen nicht alle MR-tomografisch untersucht werden. Bei einem kleinen Teil der Probanden kam es organisatorischen Gründen (z.B. Terminschwierigkeiten) nicht zur MR-Untersuchung, ein anderer Teil lehnte die MRUntersuchung wegen „Platzangst” ab oder brach sie deswegen vorzeitig ab, ein weiterer kleiner Teil der durchgeführten MR-Untersuchungen konnte wegen schlechter Bildqualität nicht ausgewertet werden (Einzelfälle).

Damit konnten bei den Bipolaren Patienten insgesamt 39 MR-Datensätze, bei den schizophren ersterkrankten Patienten insgesamt ebenfalls 35 MR-Datensätze, bei den Zwangspatienten 27 MR-Datensätze und bei der gesunden Kontrollgruppe 37 
MR-Datensätze ausgewertet werden. Mit der voxel-basierten Morphometrie (VBM) unter Zuhilfenahme des Programms SPM 2 wurde das gesamte Hirnvolumen und mit dem VOI-Tool des Programms MRICroN (siehe technische Parameter) manuell das Volumen des DLPFC rechts und links ermittelt werden. 
Tabelle 9

\begin{tabular}{|c|c|c|c|c|c|c|c|c|c|c|c|}
\hline \multicolumn{12}{|c|}{ MR-Stichproben } \\
\hline & \multicolumn{2}{|c|}{$\begin{array}{c}C \\
n=37\end{array}$} & \multicolumn{2}{|c|}{$\begin{array}{c}\mathrm{Sz} \\
\mathrm{n}=35\end{array}$} & \multicolumn{2}{|c|}{$\begin{array}{c}\text { Bip } \\
n=39\end{array}$} & \multicolumn{2}{|c|}{$\begin{array}{l}\text { OCD } \\
n=27\end{array}$} & \multirow[b]{2}{*}{$\mathrm{F}$} & \multirow[b]{2}{*}{ df } & \multirow[b]{2}{*}{$\mathrm{p}$} \\
\hline & $\mathrm{m}$ & $\mathrm{Sd}$ & $\mathrm{m}$ & sd & $\mathrm{m}$ & sd & $\mathrm{m}$ & sd & & & \\
\hline $\begin{array}{l}\text { Alter bei MRT } \\
\text { (Jahre) }\end{array}$ & 35,00 & 11,45 & 28,75 & 7,47 & 43,75 & 12,33 & 35,06 & 9,81 & 12,38 & 3,134 & $<0.0005$ \\
\hline $\begin{array}{l}\text { Schule und } \\
\text { Ausbildung } \\
\text { (Bildungsjahre) }\end{array}$ & 15,07 & 2,72 & 12,81 & 2,78 & 13,99 & 2,91 & 13,37 & 2,45 & 4,35 & 3,133 & 0,006 \\
\hline $\begin{array}{l}\text { Krankheitsdauer } \\
\text { (Jahre) }\end{array}$ & & & & & 13,92 & 10,72 & 13,67 & 9,58 & & & \\
\hline $\begin{array}{l}\text { Erstmanifestations } \\
\text {-alter (Jahre) }\end{array}$ & & & & & 28,89 & 9,50 & 21,56 & 10,87 & & & \\
\hline $\begin{array}{l}\text { Anzahl } \\
\text { depressiver } \\
\text { Episoden (max. } \\
\text { 20) }\end{array}$ & & & & & 7,57 & 7,39 & & & & & \\
\hline $\begin{array}{l}\text { Anzahl manischer } \\
\text { Episoden (max. } \\
\text { 20) }\end{array}$ & & & & & 7,57 & 8,14 & & & & & \\
\hline MADRS (Score) & & & & & 4,40 & 3,32 & & & & & \\
\hline YMRS (Score) & & & & & 2,56 & 2,82 & & & & & \\
\hline $\begin{array}{l}\text { Dauer der } \\
\text { unbehandelten } \\
\text { Psychose (DUP) } \\
\text { (Wochen) }\end{array}$ & & & 48,43 & 59,22 & & & & & & & \\
\hline $\begin{array}{l}\text { Dauer der } \\
\text { unbehandelten } \\
\text { Erkrankung (DUI) } \\
\text { (Wochen) }\end{array}$ & & & 180,03 & 165,98 & & & & & & & \\
\hline $\begin{array}{l}\text { PANSS positiv } \\
\text { Score }\end{array}$ & & & 21,40 & 6,72 & & & & & & & \\
\hline $\begin{array}{l}\text { PANSS negativ } \\
\text { Score }\end{array}$ & & & 21,94 & 6,59 & & & & & & & \\
\hline $\begin{array}{l}\text { PANSS allgemeine } \\
\text { Psychopathologie } \\
\text { Score }\end{array}$ & & & 46,83 & 10,15 & & & & & & & \\
\hline
\end{tabular}


Tabelle 10

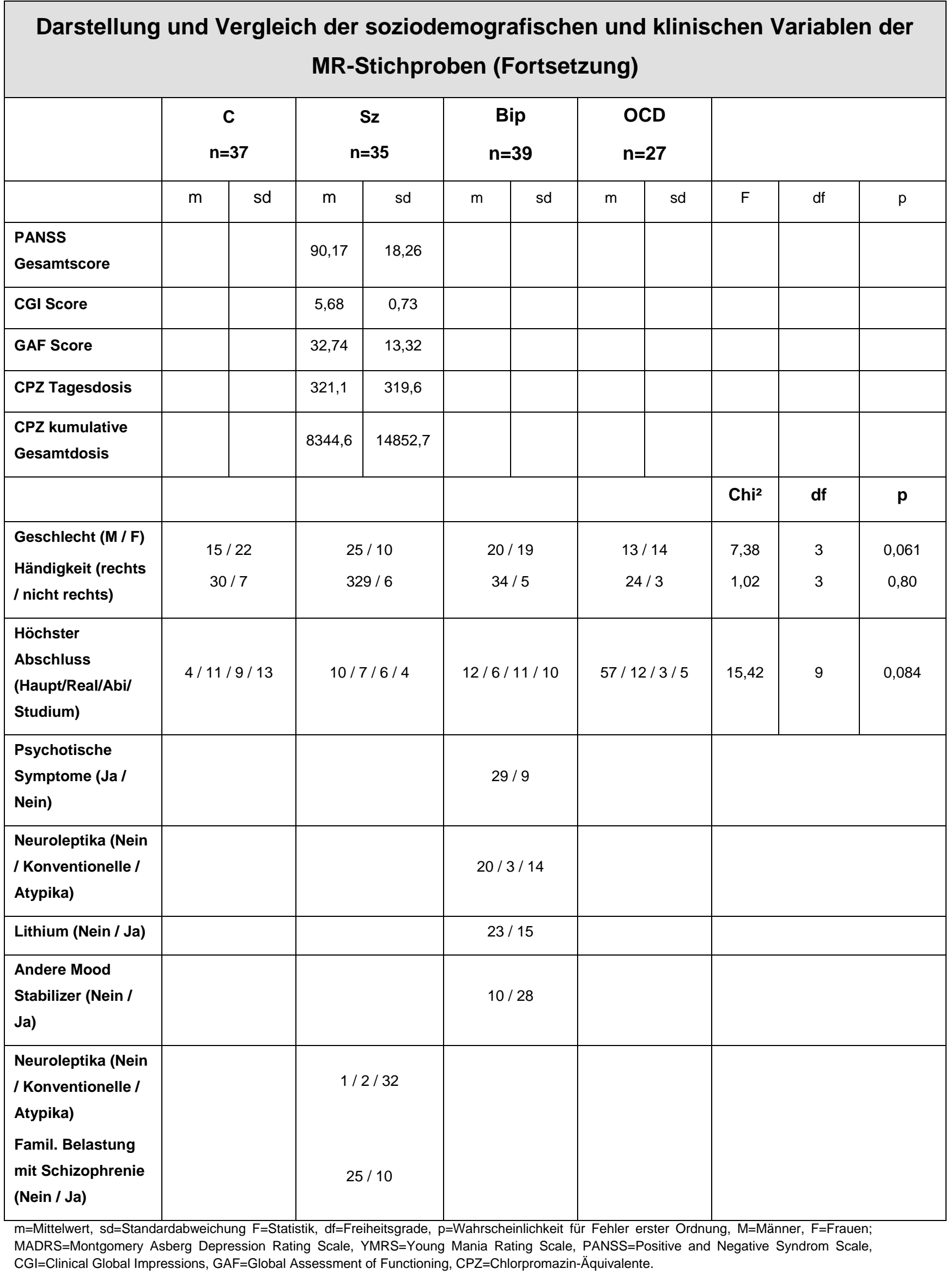


Es ergaben sich insgesamt signifikante Altersdifferenzen. Bipolare Patienten waren am ältesten, gefolgt von Zwangspatienten (OCD) und Kontrollen. Am jüngsten waren die Schizophreniepatienten. Die Bildungsdauer war zwischen den Patientengruppen signifikant verschieden. Die Kontrollgruppe wies die längste Bildungsdauer, die Schizophreniegruppe die kürzeste Bildungsdauer auf.

\subsection{Vergleich der DLPFC-Volumina zwischen den Diagnosegruppen}

Berechnet wurden Fallzahl, Mittelwert und Standardabweichung für die abhängigen Variablen (absolutes und relatives DLPFC-Volumen, Asymmetrie-Index). Dann wurde die prozentuale Differenz der Mittelwerte zwischen den Kontrollen und jeweils den 3 Patientengruppen (Schizophrene, Bipolare, OCD-Patienten) gebildet.

Die vergleichende Statistik bezüglich des Gesamthirnvolumens der grauen Substanz (GS), des Asymmetriekoeffizienten, für das absolute und das relative DLPFC zum PFC und zum Gesamthirnvolumen wurde mit Hilfe einer multivariaten Varianzanalyse (MANOVA) durchgeführt (siehe Tabelle 11).

Im Ergebnis ergaben sich signifikant unterschiedliche Volumina der absoluten Volumina DLPFC grau links, rechts und insgesamt.

Weiterhin ergaben sich sowohl für die linke graue Substanz des DLPFC als auch für die gesamte graue Substanz des DLPFC relativ zur grauen Substanz des PFC (PFCGS) signifikant unterschiedliche Volumina der Gruppen im Vergleich untereinander.

Dabei haben die Schizophrenen die größten Volumina und die Bipolaren die kleinsten Volumina im Vergleich der linken grauen Substanz des DLPFC relativ zur grauen Substanz des PFC.

Im Vergleich der gesamten grauen Substanz des DLPFC mit der grauen Substanz des PFC haben die Schizophrenen die größten Volumina und die Kontrollen die kleinsten Volumina.

Alters- und Geschlechtseffekte wurden hier nicht berücksichtigt. 
Tabelle 11

\section{Vergleich der Hirnvolumina in den Diagnosegruppen}

\begin{tabular}{|c|c|c|c|c|c|c|c|c|c|c|c|}
\hline & \multicolumn{2}{|c|}{$\begin{array}{c}C \\
(n=37)\end{array}$} & \multicolumn{2}{|c|}{$\begin{array}{c}S z \\
(n=35)\end{array}$} & \multicolumn{2}{|c|}{$\begin{array}{c}\text { Bip } \\
(n=40)\end{array}$} & \multicolumn{2}{|c|}{$\begin{array}{c}\text { OCD } \\
(n=27)\end{array}$} & \multicolumn{3}{|c|}{ MANOVA } \\
\hline & m & sd & m & sd & m & sd & $\mathbf{m}$ & sd & $\mathbf{F}$ & df & p \\
\hline Gesamtvolumen der GS $\left(\mathrm{cm}^{3}\right)$ & 731,93 & 84,75 & 755,94 & 73,68 & 726,52 & 78,66 & 724,05 & 59,45 & 2,0 & 3,134 & 0,18 \\
\hline PFC GS $\left(\mathrm{cm}^{3}\right)$ & 90,76 & 12,36 & 91,65 & 14,24 & 88,09 & 14,44 & 87.06 & 14,93 & 0,7 & 3,117 & 0,60 \\
\hline \multicolumn{12}{|l|}{ absolutes Volumen DLPFC } \\
\hline DLPFC links $\left(\mathrm{mm}^{3}\right)$ & 14791 & 2560 & 16212 & 3249 & 14086 & 2944 & 14385 & 2744 & 3,7 & 3,134 & 0,013 \\
\hline DLPFC rechts $\left(\mathrm{mm}^{3}\right)$ & 14762 & 2422 & 16475 & 2900 & 14236 & 3276 & 14396 & 2979 & 4,4 & 3,134 & 0,006 \\
\hline DLPFC gesamt $\left(\mathrm{mm}^{3}\right)$ & 29554 & 4822 & 32687 & 5844 & 283225 & 5843 & 28781 & 5588 & 4,4 & 3,134 & 0,005 \\
\hline \multicolumn{12}{|c|}{ DLPFC Volumen (relativ zum ganzen Gehirn) } \\
\hline DLPFC links grau / GS (\%) & 0,0202 & 0,0026 & 0,0212 & 0,0034 & 0,0194 & 0,0033 & 0,0199 & 0,0033 & 2,1 & 3,134 & 0,10 \\
\hline DLPFC rechts grau / GS (\%) & 0,0202 & 0,0027 & 0,0427 & 0,0055 & 0,0390 & 0,039 & 0,0199 & 0,0036 & 2,4 & 3,134 & 0,067 \\
\hline DLPFC grau / GS (\%) & 0,0404 & 0,0051 & 0,0427 & 0,0055 & 0,0390 & 0,006 & 0,0397 & 0,0066 & 2,6 & 3,134 & 0,055 \\
\hline \multicolumn{12}{|c|}{ DLPFC Volumen (relativ zum PFC) } \\
\hline DLPFC links rau / GS \% & 0,3364 & 0,0346 & 0,3637 & 0,0631 & 0,3299 & 0,0524 & 0,3507 & 0,0462 & 2,8 & 3,117 & 0,043 \\
\hline DLPFC rechts grau / GS (\%) & 0,3170 & 0,0429 & 0,3459 & 0,0429 & 0,3314 & 0,0572 & 0,3225 & 0,0494 & 2,3 & 3,117 & 0,086 \\
\hline DLPFC grau / PFC-GS & 0,3260 & 0,0332 & 0,3536 & 0,0439 & 0,3301 & 0,0469 & 0,3354 & 0,0424 & 2,8 & 3,117 & 0,044 \\
\hline $\begin{array}{l}\text { Asymmetriekoeffizient (AI) } \\
2 *(\text { r. }- \text { I. }) /(\text { r. }+ \text { I. })\end{array}$ & 0,0007 & 0,0902 & 0,0217 & 0,1201 & 0,0062 & 0,1496 & 0,0024 & 0,0855 & 0,3 & 3,134 & 0,83 \\
\hline
\end{tabular}

$\mathrm{m}=$ Mittelwert, $\mathrm{n}=$ Samplegröße, sd=Standardabweichung, $\mathrm{GS}=$ graue Substanz, $\mathrm{F}=\mathrm{F}$ Statistik, df=Freiheitsgrade, $\mathrm{p}=$ Wahrscheinlichkeit für Fehler erster Ordnung, PFC=präfrontaler Kortex, DLPFC=dorsolateraler präfrontaler Kortex

Die Subgruppenanalysen (Zwei-Gruppen-Vergleich) wurden mit Hilfe der univariaten Varianzanalyse (ANOVA) (jeweils Bipolare, Schizophrene und OCD-Patienten vs. Kontrollen) durchgeführt und für multiples Testen nach Bonferroni korrigiert (siehe nachfolgende Tabelle 12). 
Tabelle 12

\begin{tabular}{|c|c|c|c|}
\hline \multicolumn{4}{|c|}{ Vergleich der Hirnvolumina - Subgruppen-Analysen (Bonferroni) } \\
\hline & Sz vs. C & Bip vs. C & OCD vs. C \\
\hline & $\mathrm{P}$ & $\mathrm{P}$ & $\mathrm{p}$ \\
\hline graue Substanz Gesamt $\left(\mathrm{cm}^{3}\right)$ & 0,48 & 1,00 & 1,00 \\
\hline PFC graue Substanz $\left(\mathrm{cm}^{3}\right)$ & 1,00 & 1,00 & 1,00 \\
\hline \multicolumn{4}{|c|}{ absolutes Volumen DLPFC } \\
\hline DLPFC links $\left(\mathrm{mm}^{3}\right)$ & 0,23 & 1,00 & 1,00 \\
\hline DLPFC rechts $\left(\mathrm{mm}^{3}\right)$ & 0,083 & 1,00 & 1,00 \\
\hline DLPFC grau $\left(\mathrm{mm}^{3}\right)$ & 0,11 & 1,00 & 1,00 \\
\hline \multicolumn{4}{|c|}{ relatives Volumen zum gesamten Gehirn } \\
\hline DLPFC grau links / GS (\%) & 1,0 & 1,00 & 1,00 \\
\hline DLPFC grau rechts / GS (\%) & 0,55 & 1,00 & 1,00 \\
\hline DLPFC grau gesamt / GS (\%) & 0,66 & 1,00 & 1,00 \\
\hline \multicolumn{4}{|c|}{ relatives Volumen zum PFC } \\
\hline DLPFC grau links / PFC-GS & 0,15 & 1,00 & 1,00 \\
\hline DLPFC grau rechts / PFC-GS & 0,087 & 1,00 & 1,00 \\
\hline DLPFC grau gesamt / PFC-GS & 0,042 & 1,00 & 1,00 \\
\hline $\begin{array}{l}\text { Asymmetriekoeffizient } \\
2 *(\text { r. }- \text { I. }) /(\text { r. }+ \text { I. })\end{array}$ & 1,00 & 1,00 & 0,92 \\
\hline
\end{tabular}

$\mathrm{Sz}=$ Schizophrene, $\mathrm{C}=$ Kontrollen, $\mathrm{Bip}=$ Bipolare, $\mathrm{OCD}=$ Zwangserkrankte, $\mathrm{PFC}=$ präfrontaler Kortex, DLPFC=dorsolateraler präfrontaler Kortex, GS=graue Substanz

In der Subgruppenanalyse, korrigiert nach Bonferroni, ergab sich ein signifikant vergrößertes Volumen der Grauen Substanz des DLPFC relativ zur grauen Substanz des PFC bei den schizophrenen Patienten im Vergleich zu den gesunden Kontrollen. Die anderen Patientengruppen zeigten keine signifikanten Volumenunterschiede zu den gesunden Kontrollen. 


\subsection{Berücksichtigung des Einflusses intervenierender Variablen}

Mit den demographischen Daten der 4 Diagnosegruppen wurden intervallskalierte Variablen erstellt, mit denen Fallzahl, Mittelwert, Standardabweichung und Mittelwertvergleich (Faktor Diagnose) durch einfaktorielle ANOVA ermittelt wurden. Kategorielle Variablen waren die Fallzahlen der einzelnen Kategorien. Mit dem Chi²Test wurde auf Unabhängigkeit getestet.

Es ergaben sich signifikante Altersdifferenzen, Bipolare Patienten waren am ältesten, gefolgt von Zwangspatienten und Kontrollen, schizophren Ersterkrankte waren am jüngsten.

Die Bildungsdauer war zwischen den Patientengruppen signifikant verschieden (am längsten bei den Kontrollen, am kürzesten bei den schizophrenen Patienten).

Der Anteil der weiblichen Probanden war am höchsten bei den Kontrollen und am kleinsten bei den Schizophrenen, über alle 4 Diagnosegruppen war die Geschlechtsverteilung aber nicht signifikant verschieden.

Die Händigkeitsverteilung war zwischen den Diagnosegruppen nicht signifikant verschieden. Die vergleichende Statistik zwischen den Diagnosegruppen hinsichtlich der soziodemografischen Parameter wurde bereits detailliert in Abschnitt 3.2. dargestellt.

Um den Einfluss der o.g. Variablen auf das Hirnvolumen zu untersuchen, wurde eine zweifaktorielle ANOVA mit den Faktoren Diagnose und Geschlecht durchgeführt.

Es ergaben sich signifikante Geschlechtsdifferenzen für das Volumen der gesamten grauen Substanz (GS), das relative Volumen der grauen Substanz des präfrontalen Kortex (PFC-GS) und für das absolute DLPFC-Volumen. Beide waren bei den weiblichen Patienten signifikant kleiner als bei den männlichen Probanden $(p<0,006)$. Für das relative DLPFC-Volumen ergaben sich keine signifikanten Geschlechtsdifferenzen.

Weiterhin wurde eine zweifaktorielle ANOVA mit den Faktoren Diagnose und Händigkeit durchgeführt. Während sich bei rechtshändigen Probanden im Mittel fast symmetrische, d.h. gleich große DLPFC-GS-Volumina auf beiden Seiten ergaben, 
zeigte sich bei den nicht-rechtshändigen Probanden eine rechtsseitige Asymmetrie, d.h. das DLPFC-GS-Volumen war im Durchschnitt rechts größer als links $(p=0,020)$. Schaut man sich die Subgruppen numerisch an, so findet sich dieser beschriebene Unterschied nicht bei den schizophrenen Patienten.

Des weiteren wurden bivariate Korrelationen zwischen Alter und Bildung und den abhängigen Variablen nach Pearson berechnet. Im Gesamtsample korrelierte das das Alter signifikant negativ mit dem gesamten Volumen der grauen Substanz, dem PFC-GS-Volumen und den absoluten DLPFC-Volumina, in anderen Worten bedeutet dies eine Abnahme der dorsolateralen und gesamten grauen Substanz mit dem Alter. Ebenso signifikant negativ korrelierte das Alter mit den relativen DLPFCVolumen (bezogen auf die gesamte graue Substanz), d.h. der Alterseffekt bildet sich auch dann ab, wenn für die gesamte graue Substanz „normalisiert“ wird, ist also vermutlich in der Region DLPFC besonders ausgeprägt.

Im Gesamtsample korrelierte die Bildungsdauer signifikant positiv mit dem Volumen der gesamten grauen Substanz, d.h. ein größeres Volumen der grauen Substanz ging im Durchschnitt mit einer höheren Bildungsdauer (Bildungsstand) einher. Allerdings ergab sich eine signifikant negative Korrelation zwischen der Bildung und dem relativen Volumen des DLPFC bezogen auf das graue Volumen des Präfrontalkortex (DLPFC-GS-Volumen relativ zu dem PFC-GS-Volumen). Für die Kontrollgruppe alleine, korrelierte die Bildungsdauer (wie im Gesamtsample) signifikant positiv mit dem Volumen der gesamten grauen Substanz. Hier ergab sich zusätzlich eine positive Korrelation von Bildungsdauer und der grauen Substanz des präfrontalen Kortex (PFC-GS-Volumen).

Da sich im Vergleich der demografischen Variablen (Alter, Geschlecht, Bildung, Händigkeit), welche alle einen potentiellen und für Subgruppen hier gesicherten Einfluss auf das Hirnvolumen aufweisen, Unterschiede zwischen den Diagnosegruppen ergaben, wurden die intervenierenden Variablen für die weiteren Auswertungen wie folgt adjustiert.

GS und PFC-GS wurden für Alter, Bildungsdauer und Geschlecht, DLPFC-graueSubstanz-Volumina absolut für Alter und Geschlecht adjustiert. 
Weiter wurde das Alter für das DLPFC graue Substanz Volumen relativ zum GSVolumen adjustiert und die Bildungsdauer für DLPFC graue Substanz Volumen relativ zum PFC-GS-Volumen angepasst.

Der DLPFC-Asymmetriekoeffizient wurde für die Händigkeit korrigiert.

Die vergleichende Statistik unter Adjustierung der o.g. intervenierenden Variablen wurde bezüglich des Gesamthirnvolumens der grauen Substanz (GS) und des Volumens der grauen Substanz des PFC (PFC-GS) mittels univariater Kovarianzanalyse (ANCOVA mit den Faktoren Diagnose, Geschlecht, Kovariaten Alter und Bildungsdauer), für das absolute und das relative DLPFC-Volumen mit Hilfe der multivariaten Kovarianzanalyse (MANCOVA mit den Faktoren Diagnose, Geschlecht, Kovariaten Alter, Bildungsdauer) durchgeführt. Dabei wurden die Volumina relativ zum Gesamthirnvolumen der grauen Substanz (GS) und relativ zum Volumen der grauen Substanz des PFC (PFC-GS) betrachtet.

Für den DLPFC-Asymmetriekoeffizienten wurde eine multivariate Varianzanalyse (MANOVA) mit den Faktoren Diagnose und Händigkeit durchgeführt.

Die Subgruppenanalysen wurden ebenfalls mit Hilfe der multivariaten Kovarianzanalyse (MANCOVA mit den Faktoren Diagnose, Geschlecht, Kovariaten Alter, Bildungsdauer) (jeweils für Bipolare, schizophrene und Zwangspatienten vs. Kontrollen) berechnet.

Es wurde für multiples Testen nach Bonferroni korrigiert.

Aus den univariaten Analysen ergab sich für das linke DLPFC-Volumen relativ zum PFC-Volumen ein Trend zu Diagnoseeffekten $(p=0,076)$. Dabei war das relative Volumen am größten bei den schizophrenen Patienten und am kleinsten bei den Bipolaren Patienten.

In der Subgruppenanalyse Schizophrene versus Kontrollen ergab sich ebenfalls ein Trend zu erhöhtem linken DLPFC-Volumen relativ zum PFC-Volumen bei den schizophrenen Patienten $(p=0,090)$. Für das gesamte DLPFC-Volumen graue Substanz im Verhältnis zur grauen Substanz des präfrontalen Kortex (DLPFC grau/PFC-GS) ergab sich sogar ein signifikant größeres Volumen bei den schizophrenen Patienten im Vergleich zu den Kontrollen. 
Tabelle 13

\section{Einfluss von intervenierenden Variablen und Darstellung der korrigierten p-Werte nach Bonferroni-Korrektur in der Gesamt- und Subgruppen-Analyse \\ MANCOVA (Faktor Diagnose; angeglichen für Geschlecht, Alter, Bildung)}

\begin{tabular}{|c|c|c|c|c|c|c|c|c|c|c|}
\hline & $\mathbf{a}$ & b & $\mathbf{g}$ & h & \multicolumn{3}{|c|}{$\begin{array}{c}\text { Faktor } \\
\text { Diagnosegruppe }\end{array}$} & \multicolumn{3}{|c|}{$\begin{array}{c}\text { Subgruppenanalyse } \\
\text { (Bonferroni); } \\
\text { p-Werte }\end{array}$} \\
\hline & $\mathrm{p}$ & $\mathrm{p}$ & $\mathrm{p}$ & $\mathrm{P}$ & $d f$ & $\mathrm{~F}$ & $\mathrm{p}$ & $\begin{array}{l}\text { Sz vs } \\
\text { C }\end{array}$ & $\begin{array}{l}\text { Bip } \\
\text { vs C }\end{array}$ & $\begin{array}{l}\text { OCD } \\
\text { vs C }\end{array}$ \\
\hline $\begin{array}{l}\text { graue Substanz } \\
\text { total }\left(\mathrm{cm}^{3}\right) \text { (1) }\end{array}$ & $<0,0005$ & 0,069 & $<0,0005$ & & 3,127 & 0,98 & 0,40 & 1,00 & 0,23 & 1,00 \\
\hline $\begin{array}{l}\text { graue Substanz } \\
\text { PFC }\left(\mathrm{cm}^{3}\right)(1)\end{array}$ & $<0,0005$ & 0,84 & $<0,0005$ & & 3,110 & 2,53 & 0,061 & 0,18 & 0,77 & 1,00 \\
\hline \multicolumn{5}{|c|}{ DLPFC absolutes Volumen (2) } & 6,258 & 0,46 & 0,84 & & & \\
\hline DLPFC links grau $\left(\mathrm{mm}^{3}\right)$ & $<0,0005$ & & 0,006 & & 3,129 & 0,40 & 0,75 & 1,00 & 1,00 & 1,00 \\
\hline $\begin{array}{l}\text { DLPFC rechts grau } \\
\left(\mathrm{mm}^{3}\right)\end{array}$ & $<0,0005$ & & 0,011 & & 3,129 & 0,68 & 0,57 & 1,00 & 1,00 & 1,00 \\
\hline DLPFC grau $\left(\mathrm{mm}^{3}\right)$ & $<0,0005$ & & 0,005 & & 3,129 & 0,57 & 0,64 & 1,00 & 1,00 & 1,00 \\
\hline \multicolumn{5}{|c|}{ DLPFC relatives Volumen zum gesamten Gehirn (3) } & 6,266 & 0,39 & 0,89 & & & \\
\hline DLPFC links grau / GS & 0,003 & & & & 3,133 & 0,40 & 0,75 & 1,00 & 1,00 & 1,00 \\
\hline DLPFC rechts grau / GS & 0,003 & & & & 3,133 & 0,73 & 0,54 & 0,45 & 1,00 & 1,00 \\
\hline DLPFC grau / GS & 0,001 & & & & 3,133 & 0,63 & 0,60 & 0,85 & 1,00 & 1,00 \\
\hline \multicolumn{5}{|c|}{ DLPFC relatives Volumen zum PFC (4) } & 9,345 & 1,46 & 0,16 & & & \\
\hline $\begin{array}{l}\text { DLPFC links grau / PFC- } \\
\text { GS }\end{array}$ & & 0,41 & & & 3,155 & 2,35 & 0,076 & 0,090 & 1,00 & 0,75 \\
\hline $\begin{array}{l}\text { DLPFC rechts grau / } \\
\text { PFC-GS }\end{array}$ & & 0,59 & & & 3,155 & 1,47 & 0,23 & 0,081 & 1,00 & 1,00 \\
\hline DLPFC grau / PFC-GS & & 0,87 & & & 3,155 & 1,96 & 0,12 & 0,033 & 1,00 & 1,00 \\
\hline \multicolumn{11}{|l|}{ Asymmetriekoeffizient } \\
\hline $2^{\star}\left(r_{.}-\mathrm{I}_{.}\right) /\left(\mathrm{r}_{\mathrm{r}}+\mathrm{l}.\right)$ & & & & 0,62 & 3,130 & 0,1 & 0,98 & 1,00 & 1,00 & 1,00 \\
\hline
\end{tabular}

$\mathrm{a}=$ Alter, $\mathrm{b}=$ Bildung, $\mathrm{g}=$ Geschlecht, $\mathrm{h}=$ Hand, $\mathrm{F}=\mathrm{F}$ Statistik, $\mathrm{df}=$ Freiheitsgrade, $\mathrm{p}=$ Wahrscheinlichkeit für Fehler erster Ordnung,

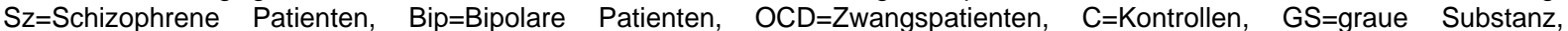
$\mathrm{PFC}=$ präfrontaler Kortex, DLPFC=dorsolateraler präfrontaler Kortex

(1) Angeglichen für Geschlecht, Alter, Bildung

(2) Angeglichen für Alter und Geschlecht

(3) Angeglichen für Bildung

(4) Angeglichen für Handpräferenz 


\subsection{Einfluss intervenierender Variablen bei Schizophrenie}

Es wurde ein nach Alter, Bildungsdauer, Geschlecht und Händigkeit weitestgehend angeglichenes Sample bestehend aus 21 Kontrollen und 21 schizophren Ersterkrankten gebildet. Aufgrund der Gesamtstichprobenstruktur war nur ein ausreichendes Matching möglich, d.h. bei den Schizophrenen war weiterhin der Anteil der männlichen Probanden höher, sie waren im Mittel jünger, und die Bildungsdauer war kürzer als bei den Kontrollen. Alle Differenzen waren nach dem Matching nicht mehr signifikant. Verglichen wurden die abhängigen Variablen zwischen den Kontrollen und den schizophren ersterkrankten Patienten im gematchten Sample. Die p-Werte wurden nicht nach Bonferroni korrigiert für multiple Tests.

Die Statistik wurde bezüglich des Gesamthirnvolumens der Grauen Substanz (GS) und des Volumens der grauen Substanz des PFC (PFC-GS) mittels univariater Kovarianzanalyse (ANCOVA mit den Faktoren Diagnose, Geschlecht, Kovariaten Alter und Bildungsdauer), für das absolute und das relative DLPFC Volumen mit Hilfe der multivariaten Kovarianzanalyse (MANCOVA mit den Faktoren Diagnose, Geschlecht, Kovariaten Alter, Bildungsdauer) durchgeführt. Dabei wurden die Volumina relativ zum Gesamthirnvolumen der graue Substanz (GS) und relativ zum Volumen der grauen Substanz des PFC (PFC-GS) betrachtet.

Für den DLPFC-Asymmetriekoeffizienten wurde eine Varianzanalyse (ANOVA mit den Faktoren Diagnose und Händigkeit) durchgeführt.

Bei schizophren Ersterkrankten versus Kontrollen ergaben sich signifikant höhere DLPFC-Volumina relativ zum PFC-GS-Volumen (links: $p=0,033$; rechts: $p=0,023$; total: $p=0,013)$. Die absoluten DLPFC-Volumina und die DLPFC-Volumina relativ zum gesamten GS-Volumen waren bei Schizophrenen im Matched Sample nicht signifikant erhöht. 
Tabelle 14

\begin{tabular}{|c|c|c|c|c|c|c|c|c|c|c|}
\hline \multicolumn{11}{|c|}{$\begin{array}{c}\text { Vergleich der abhängigen Variablen zwischen Kontrollen und sch } \\
\text { ersterkrankten Patienten im gematchten Sample } \\
\text { MANCOVA (Faktor Diagnose; adjustiert für Geschlecht, Alter, Bildung) }\end{array}$} \\
\hline & \multicolumn{2}{|c|}{$\begin{array}{l}\text { Kontrollen } \\
n=21\end{array}$} & \multicolumn{2}{|c|}{$\begin{array}{l}\text { Schizophrene } \\
\qquad n=21\end{array}$} & \multirow{2}{*}{$\frac{a}{p}$} & \multirow{2}{*}{$\begin{array}{c}\mathbf{b} \\
p\end{array}$} & \multirow{2}{*}{$\begin{array}{l}\mathbf{g} \\
p\end{array}$} & \multicolumn{3}{|c|}{$\begin{array}{l}\text { Faktor } \\
\text { Diagnosegruppe } \\
\text { (Sz vs. C) }\end{array}$} \\
\hline & $\mathrm{m}$ & sd & $\mathrm{m}$ & $\mathrm{Sd}$ & & & & df & $\mathrm{F}$ & $\mathrm{p}$ \\
\hline $\begin{array}{l}\text { graue Substanz } \\
\text { total }\left(\mathrm{cm}^{3}\right)(1)\end{array}$ & 732,320 & 96,19 & 734,40 & 68,94 & 0,002 & 0,32 & 0,060 & 1,40 & 0,05 & 0,83 \\
\hline $\begin{array}{l}\text { graue Substanz } \\
\text { PFC }\left(\mathrm{cm}^{3}\right)(1)\end{array}$ & 90,49 & 12,85 & 85,39 & 11,50 & 0,001 & 0,44 & 0,30 & 1,40 & 3,49 & 0,070 \\
\hline \multicolumn{8}{|c|}{ DLPFC absolutes Volumen (2) } & 2,36 & 0,15 & 0,86 \\
\hline $\begin{array}{l}\text { DLPFC links grau } \\
\left(\mathrm{mm}^{3}\right)\end{array}$ & 14630,7 & 2638,8 & 15137,8 & 3362,8 & 0,001 & & 0,014 & 1,37 & 0,00 & 1,00 \\
\hline $\begin{array}{l}\text { DLPFC rechts } \\
\text { grau }\left(\mathrm{mm}^{3}\right)\end{array}$ & 14652,1 & 2794,1 & 15305,2 & 2649,3 & 0,005 & & 0,059 & 1,37 & 0,11 & 0,74 \\
\hline $\begin{array}{l}\text { DLPFC grau } \\
\left(\mathrm{mm}^{3}\right)\end{array}$ & 29282,9 & 5283,5 & 30443,0 & 5762,6 & 0,001 & & 0,022 & 1,37 & 0,03 & 0,86 \\
\hline \multicolumn{8}{|c|}{ DLPFC relatives Volumen zum gesamten Gehirn (3) } & 2,38 & 0,37 & 0,69 \\
\hline $\begin{array}{l}\text { DLPFC links grau } \\
\text { / GS }\end{array}$ & 0,0200 & 0,0024 & 0,0206 & 0,0038 & 0,021 & & & 1,39 & 0,12 & 0,73 \\
\hline $\begin{array}{l}\text { DLPFC rechts } \\
\text { grau / GS }\end{array}$ & 0,0200 & 0,0027 & 0,0208 & 0,0025 & 0,15 & & & 1,39 & 0,65 & 0,42 \\
\hline DLPFC grau / GS & 0,0399 & 0,0048 & 0,0413 & 0,0059 & 0,041 & & & 1,39 & 0,37 & 0,55 \\
\hline \multicolumn{8}{|c|}{ DLPFC relatives Volumen zum PFC (3) } & 3,37 & 3,04 & 0,041 \\
\hline $\begin{array}{l}\text { DLPFC links grau } \\
\text { / PFC-GS }\end{array}$ & 0,3333 & 0,0303 & 0,3688 & 0,0731 & & 0,31 & & 1,39 & 4,91 & 0,033 \\
\hline $\begin{array}{l}\text { DLPFC rechts } \\
\text { grau / PFC-GS }\end{array}$ & 0,3145 & 0,0444 & 0,3473 & 0,0428 & & 0,61 & & 1,39 & 5,60 & 0,023 \\
\hline $\begin{array}{l}\text { DLPFC grau / } \\
\text { PFC-GS }\end{array}$ & 0,3232 & 0,0327 & 0,3567 & 0,0504 & & 0,40 & & 1,39 & 6,85 & 0,013 \\
\hline
\end{tabular}

$\mathrm{F}=$ Statistik, df=Freiheitsgrade, $\mathrm{p}=$ Wahrscheinlichkeit für Fehler erster Ordnung, Sz=Schizophrene Patienten, $C=$ Kontrollen, $a=A l t e r, b=B i l d u n g, g=G e s c h l e c h t, G S=g r a u e ~ S u b s t a n z, P F C=$ präfrontaler Kortex, DLPFC=dorsolateraler präfrontaler Kortex
(1) adjustiert für Geschlecht, Alter, Bildung
(2) adjustiert für Alter und Geschlecht
(3) adjustiert für Bildung 


\subsubsection{Einfluss familiärer Belastung bei schizophren Ersterkrankten}

Statistisch wurde eine Kovarianzanalyse durchgeführt ((M)ANCOVA) unter Berücksichtigung der intervenierenden Variablen (Kovariaten) Geschlecht, Alter und Bildungsdauer. Die folgende Auswertung bezieht sich nicht nur auf die gematchten Schizophrenen, sondern auf alle schizophren ersterkrankten Patienten der Gesamtstichprobe. 
Tabelle 15

Einfluss familiärer Belastung bei ersterkrankten Schizophreniepatienten

\begin{tabular}{|c|c|c|c|c|c|c|c|c|c|c|}
\hline & \multicolumn{3}{|c|}{$\begin{array}{l}\text { keine Schizophrenie } \\
\text { in der Familie }\end{array}$} & \multicolumn{3}{|c|}{$\begin{array}{c}\text { Schizophrenie in der } \\
\text { Familie }\end{array}$} & \multirow[t]{2}{*}{$\begin{array}{l}\text { \% Differenz } \\
\text { Ja vs. Nein }\end{array}$} & \multicolumn{3}{|c|}{$\begin{array}{l}\text { ANCOVA I } \\
\text { MANCOVA }\end{array}$} \\
\hline & $\mathrm{n}$ & $\mathrm{m}$ & sd & $\mathrm{n}$ & $\mathrm{m}$ & sd & & $d f$ & $\mathrm{~F}$ & $\mathrm{p}$ \\
\hline $\begin{array}{l}\text { graue Substanz total } \\
\left(\mathrm{cm}^{3}\right)(1)\end{array}$ & 26 & 761,0 & 82,6 & 10 & 746,1 & 63,2 & $-2,0$ & 1,30 & 0,80 & 0,38 \\
\hline $\begin{array}{l}\text { graue Substanz } \\
\text { DLPFC }\left(\mathrm{cm}^{3}\right)(1)\end{array}$ & 24 & 91,1 & 14,2 & 9 & 90,8 & 14,5 & $-0,3$ & 1,27 & 1,02 & 0,32 \\
\hline \multicolumn{8}{|l|}{ absolutes Volumen (2) } & 2,28 & 0,14 & 0,87 \\
\hline $\begin{array}{l}\text { DLPFC links grau } \\
\left(\mathrm{mm}^{3}\right)\end{array}$ & 24 & 16247,8 & 3224,8 & 10 & 15481,6 & 2843,5 & $-4,7$ & 1,29 & 0,23 & 0,64 \\
\hline $\begin{array}{l}\text { DLPFC rechts grau } \\
\left(\mathrm{mm}^{3}\right)\end{array}$ & 24 & 16464,5 & 2849,0 & 10 & 15901,1 & 2585,6 & $-3,4$ & 1,29 & 0,25 & 0,62 \\
\hline DLPFC grau $\left(\mathrm{mm}^{3}\right)$ & 24 & 32712,4 & 5677,7 & 10 & 31382,7 & 5247,5 & $-4,1$ & 1,29 & 0,29 & 0,60 \\
\hline \multicolumn{8}{|c|}{ relatives Volumen zum gesamten Gehirn (3) } & 2,30 & 0,09 & 0,92 \\
\hline $\begin{array}{l}\text { DLPFC links grau / } \\
\text { GS }\end{array}$ & 24 & 0,0211 & 0,0035 & 10 & 0,0207 & 0,0030 & $-2,0$ & 1,31 & 0,17 & 0,68 \\
\hline $\begin{array}{l}\text { DLPFC rechts grau / } \\
\text { GS }\end{array}$ & 24 & 0,0214 & 0,0026 & 10 & 0,0212 & 0,0021 & $-0,9$ & 1,31 & 0,11 & 0,75 \\
\hline DLPFC grau / GS & 24 & 0,0425 & 0,0055 & 10 & 0,0419 & 0,0048 & $-1,5$ & 1,31 & 0,17 & 0,68 \\
\hline \multicolumn{8}{|c|}{ relatives Volumen zum PFC (4) } & 2,30 & 0,74 & 0,49 \\
\hline $\begin{array}{l}\text { DLPFC links grau / } \\
\text { PFC-GS }\end{array}$ & 22 & 0,372 & 0,070 & 9 & 0,343 & 0,039 & $-7,8$ & 1,31 & 0,28 & 0,60 \\
\hline $\begin{array}{l}\text { DLPFC rechts grau / } \\
\text { PFC-GS }\end{array}$ & 22 & 0,347 & 0,046 & 9 & 0,344 & 0,035 & $-0,6$ & 1,31 & 1,41 & 0,25 \\
\hline DLPFC grau / GS & 22 & 0,358 & 0,050 & 9 & 0,343 & 0,022 & $-4,3$ & 1,31 & 0,76 & 0,39 \\
\hline $\begin{array}{l}\text { Asymmetriekoeffizient } \\
2^{*}(\text { r.-l. }) /(\text { r. }+ \text { I. })(5)\end{array}$ & 24 & 0,020 & 0,134 & 10 & 0,029 & 0,093 & & 1,30 & 0,01 & 0,90 \\
\hline
\end{tabular}

$\mathrm{n}=$ Samplegröße, m=Mittelwert, sd=Standardabweichung, \%Diff=Differenz in Prozent, F=F Statistik, df=Freiheitsgrade, $p=$ Wahrscheinlichkeit für Fehler erster Ordnung, GS=graue Substanz, PFC=präfrontaler Kortex, DLPFC=dorsolateraler präfrontaler Kortex
(1) adjustiert für Geschlecht, Alter und Bildung
(2) adjustiert für Alter und Geschlecht
(3) adjustiert für Alter
(4) adjustiert für Bildung
(5) adjustiert für Handpräferenz 
Es ergaben sich keine signifikanten Differenzen der absoluten oder relativen DLPFCVolumina zwischen Schizophrenen mit schizophren erkrankten Familienangehörigen und Patienten ohne familiär Erkrankte.

\subsubsection{Einfluss des Krankheitsverlaufs und der Psychopathologie bei schizophren Ersterkrankten}

Für die statistische Auswertung wurden partielle Korrelationen adjustiert für Alter, Bildung, Geschlecht und Händigkeit nach den oben angegebenen Analysen ausgewertet. Die Auswertung wurde zwischen schizophren Ersterkrankten, ihren Krankheitsverlaufsdaten und der Psychopathologie zum Zeitpunkt der Aufnahme in die stationäre Behandlung und den abhängigen Variablen vorgenommen. Die Korrelationen wurden nicht nach Bonferroni korrigiert. 
Tabelle 16

\begin{tabular}{|c|c|c|c|c|c|c|c|c|c|c|}
\hline \multicolumn{11}{|c|}{$\begin{array}{l}\text { Schizophren Ersterkrankte: Partielle Korrelationen zwischen } \\
\text { Krankheitsverlaufsdaten und Psychopathologie } \\
\text { ANCOVA (Faktor Diagnose; angeglichen für Geschlecht, Alter, Bildung, Händigkeit) }\end{array}$} \\
\hline & & $\begin{array}{l}\text { DUP } \\
(w)\end{array}$ & $\begin{array}{l}\text { DUI } \\
\text { (w) }\end{array}$ & $\begin{array}{c}\text { PANSS } \\
+\end{array}$ & $\begin{array}{c}\text { PANSS } \\
-\end{array}$ & $\begin{array}{l}\text { PANSS } \\
\text { general }\end{array}$ & $\begin{array}{l}\text { PANSS } \\
\text { Total }\end{array}$ & $\begin{array}{l}\text { P3 } \\
\text { Hallu } \\
\text { zinat }\end{array}$ & CGI & GAF \\
\hline $\begin{array}{l}\text { graue } \\
\text { Substanz } \\
\text { total }\left(\mathrm{cm}^{3}\right)(1)\end{array}$ & $\begin{array}{r}\mathrm{pc} \\
\mathrm{p} \\
\mathrm{df}\end{array}$ & $\begin{array}{r}-0,09 \\
0,64 \\
30\end{array}$ & $\begin{array}{r}0,07 \\
0,72 \\
30\end{array}$ & $\begin{array}{r}-0,26 \\
0,15 \\
30\end{array}$ & $\begin{array}{r}0,35 \\
0,048 \\
30\end{array}$ & $\begin{array}{r}0,13 \\
0,49 \\
30\end{array}$ & $\begin{array}{r}0,10 \\
0,58 \\
30\end{array}$ & $\begin{array}{r}-0,34 \\
0,055 \\
30\end{array}$ & $\begin{array}{r}0,02 \\
0,90 \\
29\end{array}$ & $\begin{array}{r}-0,04 \\
0,82 \\
29\end{array}$ \\
\hline $\begin{array}{l}\text { graue } \\
\text { Substanz PFC } \\
\left(\mathrm{cm}^{3}\right)(1)\end{array}$ & $\begin{array}{r}\mathrm{pc} \\
\mathrm{p} \\
\mathrm{df}\end{array}$ & $\begin{array}{r}-0,05 \\
0,79 \\
26\end{array}$ & $\begin{array}{r}-0,01 \\
0,97 \\
26\end{array}$ & $\begin{array}{r}-0,23 \\
0,24 \\
26\end{array}$ & $\begin{array}{r}0,44 \\
0,019 \\
26\end{array}$ & $\begin{array}{r}0,20 \\
0,32 \\
26\end{array}$ & $\begin{array}{r}0,18 \\
0,37 \\
26\end{array}$ & $\begin{array}{r}-0,31 \\
0,10 \\
26\end{array}$ & $\begin{array}{r}0,05 \\
0,80 \\
25\end{array}$ & $\begin{array}{r}-0,14 \\
0,49 \\
25\end{array}$ \\
\hline $\begin{array}{l}\text { DLPFC links } \\
\left(\mathrm{mm}^{3}\right)(2)\end{array}$ & $\begin{array}{l}\mathrm{pc} \\
\mathrm{p} \\
\mathrm{df}\end{array}$ & $\begin{array}{r}-0,11 \\
0,53 \\
31\end{array}$ & $\begin{array}{r}0,10 \\
0,59 \\
31\end{array}$ & $\begin{array}{r}-0,36 \\
0,037 \\
31\end{array}$ & $\begin{array}{r}0,14 \\
0,45 \\
31\end{array}$ & $\begin{array}{r}-0,06 \\
0,72 \\
31\end{array}$ & $\begin{array}{r}-0,12 \\
0,52 \\
31\end{array}$ & $\begin{array}{r}-0,26 \\
0,15 \\
31\end{array}$ & $\begin{array}{r}-0,12 \\
0,51 \\
30\end{array}$ & $\begin{array}{r}0,05 \\
0,80 \\
30\end{array}$ \\
\hline $\begin{array}{l}\text { DLPFC rechts } \\
\left(\mathrm{mm}^{3}\right)(2)\end{array}$ & $\begin{array}{l}\mathrm{pc} \\
\mathrm{p} \\
\mathrm{df}\end{array}$ & $\begin{array}{r}-0,11 \\
0,54 \\
31\end{array}$ & $\begin{array}{r}0,04 \\
0,82 \\
31\end{array}$ & $\begin{array}{r}-0,20 \\
0,26 \\
31\end{array}$ & $\begin{array}{r}0,13 \\
0,47 \\
31\end{array}$ & $\begin{array}{r}0,07 \\
0,68 \\
31\end{array}$ & $\begin{array}{r}0,02 \\
0,93 \\
31\end{array}$ & $\begin{array}{r}-0,08 \\
0,64 \\
31\end{array}$ & $\begin{array}{r}-0,02 \\
0,91 \\
30\end{array}$ & $\begin{array}{r}0,06 \\
0,75 \\
30\end{array}$ \\
\hline $\begin{array}{l}\text { DLPFC }\left(\mathrm{mm}^{3}\right) \\
(2)\end{array}$ & $\begin{array}{l}\mathrm{pc} \\
\mathrm{p} \\
\mathrm{df}\end{array}$ & $\begin{array}{r}-0,12 \\
0,51 \\
31\end{array}$ & $\begin{array}{r}0,08 \\
0,67 \\
31\end{array}$ & $\begin{array}{r}-0,31 \\
0,078 \\
31\end{array}$ & $\begin{array}{r}0,15 \\
0,42 \\
31\end{array}$ & $\begin{array}{r}0,00 \\
0,99 \\
31\end{array}$ & $\begin{array}{r}-0,06 \\
0,75 \\
31\end{array}$ & $\begin{array}{r}-0,19 \\
0,30 \\
31\end{array}$ & $\begin{array}{r}-0,08 \\
0,67 \\
30\end{array}$ & $\begin{array}{r}0,06 \\
0,75 \\
30\end{array}$ \\
\hline $\begin{array}{l}\text { DLPFC links I } \\
\text { GS (3) }\end{array}$ & $\begin{array}{l}\mathrm{pc} \\
\mathrm{p} \\
\mathrm{df}\end{array}$ & $\begin{array}{r}-0,06 \\
0,72 \\
32\end{array}$ & $\begin{array}{r}0,09 \\
0,62 \\
32\end{array}$ & $\begin{array}{r}-0,29 \\
0,09 \\
32\end{array}$ & $\begin{array}{r}0,01 \\
0,94 \\
32\end{array}$ & $\begin{array}{r}-0,12 \\
0,51 \\
32\end{array}$ & $\begin{array}{r}-0,17 \\
0,34 \\
32\end{array}$ & $\begin{array}{r}-0,07 \\
0,68 \\
32\end{array}$ & $\begin{array}{r}-0,16 \\
0,37 \\
31\end{array}$ & $\begin{array}{r}0,09 \\
0,61 \\
31\end{array}$ \\
\hline $\begin{array}{l}\text { DLPFC rechts I } \\
\text { GS (3) }\end{array}$ & $\begin{array}{l}\mathrm{pc} \\
\mathrm{p} \\
\mathrm{df}\end{array}$ & $\begin{array}{r}-0,07 \\
0,71 \\
32\end{array}$ & $\begin{array}{r}0,03 \\
0,89 \\
32\end{array}$ & $\begin{array}{r}-0,13 \\
0,47 \\
32\end{array}$ & $\begin{array}{r}-0,04 \\
0,84 \\
32\end{array}$ & $\begin{array}{r}0,01 \\
0,96 \\
32\end{array}$ & $\begin{array}{r}-0,06 \\
0,76 \\
32\end{array}$ & $\begin{array}{r}0,18 \\
0,32 \\
32\end{array}$ & $\begin{array}{r}-0,07 \\
0,69 \\
31\end{array}$ & $\begin{array}{r}0,14 \\
0,43 \\
31\end{array}$ \\
\hline DLPFC / GS (3) & $\begin{array}{l}p c \\
p \\
d f\end{array}$ & $\begin{array}{r}-0,07 \\
0,70 \\
32\end{array}$ & $\begin{array}{r}0,07 \\
0,70 \\
32\end{array}$ & $\begin{array}{r}-0,24 \\
0,17 \\
32\end{array}$ & $\begin{array}{r}-0,01 \\
0,96 \\
32\end{array}$ & $\begin{array}{r}-0,07 \\
0,70 \\
32\end{array}$ & $\begin{array}{r}-0,13 \\
0,46 \\
32\end{array}$ & $\begin{array}{r}0,04 \\
0,83 \\
32\end{array}$ & $\begin{array}{r}-0,13 \\
0,46 \\
31\end{array}$ & $\begin{array}{r}0,13 \\
0,49 \\
31\end{array}$ \\
\hline
\end{tabular}


Tabelle 17

\begin{tabular}{|l|l|r|r|r|r|r|r|r|r|r|}
\hline \multicolumn{8}{|c|}{ Schizophren Ersterkrankte: Partielle Korrelationen zwischen } \\
Krankheitsverlaufsdaten und Psychopathologie (Fortsetzung) \\
MANCOVA (Faktor Diagnose; angeglichen für Geschlecht, Alter, Bildung, Händigkeit) \\
\hline
\end{tabular}

DUP=Dauer der unbehandelten Psychose, DUI=Dauer der unbehandelten Erkrankung, w=Wochen, PANSS=Positiv-undNegativ-Syndrom-Skala, P3 Halluzinat=P3 Halluzinationen, CGI=Clinical Global Impression Score, GAF=Global Assessment of Function, $\mathrm{Pc}=$ partielle Korrelation, $\mathrm{p}=$ Wahrscheinlichkeit für Fehler erster Ordnung, df=Freiheitsgrade, $\mathrm{PFC}=$ präfrontaler Kortex, DLPFC=dorsolateraler präfrontaler Kortex

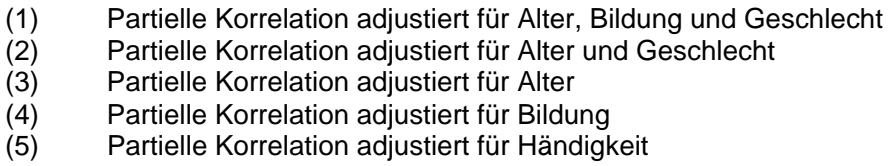

Für die schizophren Ersterkrankten ergaben sich signifikant negative Korrelationen zischen DLPFC-Volumina relativ zum PFC-GS-Volumen und PANSS allgemeine Psychopathologie sowie total und CGI. Signifikant positive Korrelationen ergaben sich zwischen DLPFC-Volumina relativ zum PFC-GS-Volumen und GAF. Dies bedeutet damit, dass je geringer der Krankheitsschweregrad und je besser das Funktionsniveau, desto höher ist das relative DLPFC-Volumen. 


\subsection{Einfluss intervenierender Variablen bei Bipolaren Patienten}

Es wurde ein nach Alter, Bildungsdauer, Geschlecht und Händigkeit weitestgehend angeglichenes Sample bestehend aus 23 Kotrollen und 23 Bipolaren Patienten gebildet. Auch nach dem Matching war das mittlere Alter bei den Bipolaren Patienten noch erhöht und die Bildungsdauer reduziert, alle Differenzen bezüglich der demographischen Variablen waren aber nach dem Matching nicht mehr signifikant.

Zwischen den Bipolaren und den gematchten Kontrollen ergaben sich keine signifikanten Mittelwertunterschiede oder Trends für das absolute oder relative DLPFC-Volumen.

\subsubsection{Einfluss des Krankheitsverlaufs bei Bipolaren Patienten}

Die partiellen Korrelationen wurde adjustiert für Alter, Bildungsdauer, Geschlecht und Händigkeit (vgl. Konsequenz für weitere Auswertungen) zwischen Krankheitsdauer, Ersterkrankungsalter und Anzahl depressiver und manischer Episoden (jeweils max. 20). Die Werte wurden mittels MADRS und YMRS und den abhängigen Variablen erhoben. Die Korrelationen sind nicht Bonferroni korrigiert.

MADRS korrelierte signifikant negativ mit dem PFC-GS-Volumen. Zu den DLPFCVolumina ergaben sich keine signifikanten Korrelationen.

\subsection{Einfluss intervenierender Variablen bei Zwangspatienten}

Es wurde ein nach Alter, Bildungsdauer, Geschlecht und Händigkeit weitestgehend angeglichenes Sample bestehend aus 26 Kontrollen und 26 Zwangspatienten gebildet. Auch nach dem Matching war die mittlere Bildungsdauer bei den Zwangspatienten noch reduziert, aber alle Differenzen bezüglich der demographischen Variablen waren nach dem Matching nicht mehr signifikant.

Auch im gematchten Sample ergaben sich für alle abhängigen Variablen zwischen Zwangspatienten und Kontrollen keine signifikanten Unterschiede. 


\subsubsection{Einfluss des Krankheitsverlaufs bei Zwangspatienten}

Die partiellen Korrelationen wurden adjustiert für Alter, Bildungsdauer, Geschlecht und Händigkeit (vgl. Konsequenz für weitere Auswertungen) zwischen Krankheitsdauer, Ersterkrankungsalter und den abhängigen Variablen. Die Korrelationen sind nicht Bonferroni-korrigiert.

Es ergab sich eine signifikant positive Korrelation zwischen der Krankheitsdauer und dem GS-Gesamthirnvolumen p.c. $=0,42, p=0,042$.

Zwischen DLPFC-Volumen und Krankheitsdauer/Ersterkrankungsalter ergaben sich keine signifikanten Korrelationen. 


\section{Diskussion}

Diese Arbeit befasst sich mit volumetrischen Untersuchungen des präfrontalen dorsolateralen Kortex (DLPFC) auf MRT-Aufnahmen. Das Patientenkollektiv bestand aus ersterkrankten schizophrenen Patienten, Bipolaren Patienten, Zwangspatienten sowie gesunden Kontrollpersonen.

Viele MR-volumetrische Studien untersuchten morphologische Veränderungen an schizophrenen Patienten im Vergleich zu einer gesunden Kontrollgruppe, diagnoseübergreifende Untersuchungen wie in dieser Arbeit wurden bisher nicht durchgeführt.

In einer Metaanalyse von Lawrie und Abukmeil (1998) wurden 40 MRT-Studien an schizophrenen Patienten im Zeitraum 1986 bis 1996 zusammengefasst. Es konnte, in einem großen Teil dieser Studien, ein vermindertes Gesamthirnvolumen bei Schizophrenen festgestellt werden. Die gemittelte Reduktion betrug 3\%. Auch wurde in 60\% der Studien eine Verkleinerung des Frontallappens um 5\% festgestellt.

Weitere Reduktionen wurden im Temporallappen, der Amygdala-Hippocampusregion, im Parietallappen, im Okzipitallappen und im Cerebellum gefunden.

In einem Überblick über strukturelle Magnetresonanztomographie bei Schizophrenie von Schmitt et al. (2001) ergab sich für den Frontallappen eine Verminderung von 70\%. Die Verminderung im Cerebellum betrug 60\%, im Thalamus 63\%. Das Gesamtvolumen des Gehirns bei schizophrenen Patienten blieb in 57\% aller Studien unverändert gegenüber gesunden Kontrollen. Bei 43\% der Patienten ließ sich jedoch eine Abnahme des Gehirnvolumens feststellen. In 83\% aller Studien wurde eine Abnahme der grauen Substanz gezeigt.

Die hier vorgelegte Arbeit beschäftigt sich mit einem Abschnitt des Frontallappens, dem dorsolateralen präfrontalen Kortex (DLPFC). Besondere Beachtung findet dieser Kortex durch die Lokalisation des Arbeitsgedächtnisses, in welchem schizophren Erkrankte Defizite aufweisen. Wie oben beschrieben befassten sich bereits zahlreiche Studien mit Volumenreduktionen des Frontallappens, zumeist wurde dabei ein voxelweiser Vergleich zwischen Erkrankten und Gesunden durchgeführt (VBM-Ansatz). In der hiesigen Arbeit wurde nach dem Region-of-Interest-Ansatz 
vorgegangen, bei dem (manuell) die interessierende Hirnregion umfahren bzw. markiert und das Volumen dieser Region bestimmt wird. Hierfür wurde das Programms MRICroN benutzt.

Der DLPFC wurde in den oben beschriebenen Grenzen beidseits manuell gemessen, um auch evtl. existierende Asymmetrien zu entdecken.

Die hohe Reliabilität der Messungen und damit auch der Zuverlässigkeit der Grenzfestlegung konnte anhand von jeweils 10 zufällig gewählten Fällen, welche verblindet doppelt markiert wurden, mit der Berechnung einer hohen Retest- und Interraterreliabilität bestätigt werden.

Um auch Aussagen über relative Größenveränderungen des DLPFC machen zu können, wurden neben diesen Daten in der statistischen Auswertung ebenfalls bereits vorhandene Daten über die Volumina des Gesamthirnvolumens und des Präfrontalkortex verwendet, welche mit dem voxelbasierten Ansatz von SPM bestimmt wurden.

Unsere Untersuchungen waren geleitet von folgenden Fragestellungen:

1. Besteht ein Unterschied (Verminderung) der Volumina des dorsolateralen präfrontalen Kortex bei Schizophrenie im Vergleich zu einer gesunden Kontrollgruppe?

2. Ist ein evtl. Unterschied (Reduktion) diagnosespezifisch, also auch im Vergleich zu einer Gruppe von Bipolaren und Zwangspatienten nachzuweisen?

Unsere Ergebnisse lassen sich folgendermaßen zusammenfassen:

- Es ergab sich ein signifikant vergrößertes Volumen der grauen Substanz des DLPFC relativ zur grauen Substanz des PFC bei den schizophrenen Patienten im Vergleich zu den gesunden Kontrollen.

- Die Bipolaren- und Zwangspatienten zeigten keine signifikanten Volumenunterschiede zu den gesunden Kontrollen. 
Diese Ergebnisse sowie die anderen Resultate sollen im Folgenden unter Bezugnahme auf die Hypothesen sowie auf den aktuellen Forschungskontext detailliert betrachtet werden.

Hierzu wurde eine systematische Literatursuche in der Datenbank „PubMed“ vorgenommen. Die hierfür bei der ersten Suche zu Beginn der Arbeit verwendeten Stichwörter waren: „dorsolateral prefrontal cortex“, „Schizophrenia“ und „structural MRI“. Nach Abschluss der Messungen fand eine zweite Recherche mit den Stichwörtern „first-episode schizophrenia" und "structural MRI" ebenfalls bei „PubMed“ statt. Die Suche ergab 350 Treffer. Auf Grund dieser sehr hohen Anzahl an so gefundenen Studien wurde die Suche weiter eingegrenzt.

Ausgehend von der Metaanalyse von Steen et al. (2006) beschränkten wir unsere Suche unter folgenden Suchwörtern auf die letzten fünf Jahre, Artikel in Englisch oder Deutsch und Studien nur an Erwachsenen. Diese Suche ergab 44 Treffer.

Die relevanten Studien aus dieser Literaturrecherche bezogen auf den präfrontalen Kortex sind in Tabelle 18 zusammengefasst. Dargestellt wurden die jeweils nur für den präfrontalen Kortex relevanten Ergebnisse, auch wenn die Studie weitere Ergebnisse für andere Hirnregionen enthielt.

Steen et al. (2006) legten ihrer Metaanalyse ebenfalls eine Literatursuche bei „PubMed“ zu Grunde. Eine erste Suche unternahmen sie unter den Stichwörtern „schizophrenia and first-episode and magnetic resonance imaging and volume“. In einem zweiten Schritt fügten sie die Schlüsselwörter „drug naive for first episode“ hinzu. Ihre Suche beschränkte sich auf die Literatur bis Ende November 2004.

Die größten Volumenunterschiede zwischen ersterkrankten Schizophrenen und gesunden Kontrollen sind nach Steen et al. (2006) in der Reduzierung der gesamten grauen Substanz und in der Vergrößerung der Ventrikelräume zu finden. Offen bleibt die Frage, ob die Reduzierung der grauen Substanz bei ersterkrankten Schizophrenen im Verlauf durch das Fortschreiten der Erkrankung zunimmt oder durch andere Faktoren, wie einer antipsychotischen Medikation, Alkoholismus, Drogenmissbrauch, Mangelernährung oder einer sozialen Deprivation bedingt ist. Einige Hirnstrukturen zeigen bereits bei Diagnosestellung eine Reduzierung der Volumina, welche sich im Verlauf der Erkrankung fortsetzt. So ist die Vergrößerung 
der lateralen Ventrikel bereits bei Erstaufnahme signifikant und setzt sich im weiteren Krankheitsverlauf fort. Auch zeigen der Hippocampus, die kortikale graue Substanz, der Heschl-Gyrus und das Planum temporale bei Diagnosestellung und beim Fortschreiten der Erkrankung einen kontinuierlichen Volumenverlust. Andere Strukturen zeigen verminderte Volumina bei Diagnosestellung, jedoch nicht im weiteren Verlauf der Erkrankung, dazu gehören der Thalamus und die Inselregion (Steen et al. 2006). Dies könnte bedeuten, dass Volumenverluste in diesen beiden Strukturen nicht fortschreitend sind oder auch nur, dass zu wenige Studien vorliegen, die diesen Verlauf untersucht haben bzw. einen Volumenverlust signifikant erscheinen lassen. 
Tabelle 18

\begin{tabular}{|c|c|c|}
\hline \multicolumn{3}{|c|}{$\begin{array}{r}\text { Ergebnisse neuerer volumetrischer MR-Studien bezüglich } \\
\text { ersterkrankten Schizophrenen }\end{array}$} \\
\hline Autor/Methode & Region & Ergebnis \\
\hline Whitford et al. 2005, VBM & $\begin{array}{l}\text { ventraler präfrontaler Kortex } \\
\text { (GS) }\end{array}$ & $\begin{array}{l}\text { reduziertes Volumen links } \\
\text { (FES vs. C) }\end{array}$ \\
\hline Farrow et al. 2005, VBM & Frontalkortex (GS) & $\begin{array}{l}\text { reduzierte Volumina bds. } \\
\text { (FES vs. C) } \\
\text { weiter reduzierte Volumina (FES } \\
\text { vs. FES follow up nach } 2 \text { Jahren) }\end{array}$ \\
\hline $\begin{array}{l}\text { Lopez-Garcia et al. 2006, ROI } \\
\text { (automatische Methode, u.a. } \\
\text { präfrontale Brodmann-Areale) }\end{array}$ & $\begin{array}{l}\text { Brodmann Areale } 8,9,45,46 \\
\text { (GS) }\end{array}$ & $\begin{array}{l}\text { links reduziertes Volumen BA } 46 \\
\text { (FES vs. C) } \\
\text { links reduziertes Volumen BA } 46 \\
\text { (CS vs. FES) }\end{array}$ \\
\hline Molina et al. 2006, ROI & DLPFC, GTS & $\begin{array}{l}\text { rechts reduziertes Volumen (FES } \\
\text { vs. FENS bzw. C) }\end{array}$ \\
\hline $\begin{array}{l}\text { Zipparo et al. 2007, ROI } \\
\text { (automatisch, Maske) }\end{array}$ & Frontallappen (GS) & $\begin{array}{l}\text { reduzierte Volumina bds. } \\
\text { (FES vs. FES follow up nach 2-3 } \\
\text { Jahren) }\end{array}$ \\
\hline $\begin{array}{l}\text { Schaufelberger et al. } 2007 \text {, } \\
\text { VBM }\end{array}$ & linker präfrontaler Kortex (GS) & $\begin{array}{l}\text { reduzierte Volumina bds. } \\
\text { (FES vs. C) }\end{array}$ \\
\hline Meisenzahl et al. 2008, VBM & $\begin{array}{l}\text { Orbitofrontaler Kortex (GS) } \\
\text { DLPFC (GS) } \\
\text { Gyrus rectus (GS) } \\
\text { dorsomedialer Präfrontalkortex } \\
\text { (GS) }\end{array}$ & $\begin{array}{l}\text { reduzierte Volumina bds. } \\
\text { (FES vs. C) }\end{array}$ \\
\hline $\begin{array}{l}\text { Whitford et al. 2009, VBM } \\
\text { (vorher Clusterkorrelation mit } \\
\text { Item P1 aus der PANSS-Wahn) }\end{array}$ & $\begin{array}{l}\text { dorsomedialer Frontalkortex } \\
\text { (GS) links }\end{array}$ & $\begin{array}{l}\text { reduziertes Volumen des „Wahn- } \\
\text { Clusters ( } 54 \text { Voxel)“ } \\
\text { (FES vs. C) }\end{array}$ \\
\hline $\begin{array}{l}\text { Takayanagi et al. 2010, ROI } \\
\text { (manuell) }\end{array}$ & $\begin{array}{l}\text { Gyrus frontalis medialis, Gyrus } \\
\text { frontalis superior (GS) }\end{array}$ & $\begin{array}{l}\text { kein signifikanter } \\
\text { Volumenunterschied (FES vs. C) }\end{array}$ \\
\hline
\end{tabular}


Fast alle der erwähnten Studien beschreiben Volumenreduktionen im Bereich des präfrontalen Kortex. Lediglich Takayanagi et al. 2010 konnten keinen signifikanten Volumenunterschied der schizophren Ersterkrankten im Vergleich zu gesunden Kontrollen feststellen. Farrow et al. (2005) und Zipparo et al. (2007) führten follow up Untersuchungen nach 2-3 Jahren durch und konnten weitere Volumenverluste in den oben benannten Arealen bei Ihren Patienten darstellen. Die Verschlechterung kognitiver Funktionen bei schizophren Ersterkrankten geht also mit einem Verlust von Volumen in den beschriebenen Regionen einher (Zipparo et al. 2007).

Kritisch zu betrachten sind die unterschiedlichen Methoden der Autoren. Einige Autoren nutzen die voxel-basierte Morphometrie, andere untersuchten die Areale anhand der ROI-Methode (region of interest). Dabei wurden jedoch zumeist nicht, wie in unserer Arbeit, die Areale einzeln manuell markiert, sondern mit einer automatischen Methode bestimmt. Dafür stellten Lopez-Garcia et al. (2006), Molina et al. (2006) und Zipparo et al. (2007) Masken der zu messenden Region anhand festgelegter Koordinaten standardisierter Atlanten (Talairach und Tournoux 1988) her und projizierten die MRTs der Patienten auf ein sogenanntes Standardgehirn. Die Vermessung der Areale und Bestimmung der Volumina erfolgte dann automatisch. Diese Methodik lässt sich mit der manuellen Segmentierung, wie sie in dieser Arbeit erfolgte, nicht vergleichen, da sie individuelle anatomische Gegebenheiten nur unzureichend berücksichtigt.

Lediglich Takayanagi et al. (2010) wählten eine ROI basierte manuelle Methode, führten die Messungen allerdings nur an 3 koronaren Schichten durch und wählten die Schichten posterior des Balkenknies aus. Wie wir orientierten sie sich in ihrer Grenzziehung des frontalen Kortex an Crespo-Facorro et al. (1999).

\subsection{Kritische Wertung methodischer Aspekte}

Kritisch betrachtet werden muss zunächst die Festlegung der anatomischen Grenzen des DLPFC in dieser Arbeit. Besondere Bedeutung hatte für uns die Lokalisation des Arbeitsgedächtnisses auf diesem Kortex.

In neuropsychologischen Studien mit schizophrenen Patienten konnten häufig Defizite im Bereich der sogenannten exekutiven Funktionen gefunden werden 
(Robbins 1990, Weinberger et al. 1994, Elliott et al. 1995). Darunter werden jene kognitiven Prozesse verstanden, die der Handlungssteuerung und Handlungskontrolle dienen und in unterschiedlichsten Modellen als wesentliche Komponenten menschlicher Informationsverarbeitung und Handlungssteuerung beschrieben werden (Matthes-von Cramon \& Cramon 2000, Baddeley 1992, Goldman-Rakic 1992). Eine besondere Bedeutung bei der Handlungsplanung und Handlungskontrolle kommt dabei dem Arbeitsgedächtnis zu, einem System, das zuständig ist für die vorübergehende Speicherung und gleichzeitige Verarbeitung von Informationen und als zentral für Verhalten und Kognitionen des Menschen angesehen wird. Da diese Funktionen bei schizophrenen Patienten insbesondere beeinträchtigt sind, sind ggf. auch morphometrische Veränderungen dieser Region zu erwarten.

Frühere Studien beschäftigen sich vor allem mit größeren Abschnitten des Präfrontalkortex, wenig jedoch mit der Substruktur des DLPFC alleine. Wir legten die Ausdehnung des DLPFC auf den Gyrus frontalis superior und den Gyrus frontalis medius fest.

Im Gegensatz zu Crespo-Facorro et al. (1999), die den Beginn des Gyrus frontalis medius am inneren Knie des Corpus callosum festmachen, entschieden wir uns, unsere Messungen am äußeren/vorderen Knie des Corpus callosum zu beginnen, da in vielen Arbeiten wie u.a. in Falkai et al. (2004) das vordere Knie des Corpus callosum als dorsale Grenze des Präfrontalkortex festgelegt wurde.

Die Endschicht, sowie die laterale und mediale Grenze des DLPFC wurden mit Hilfe der in der Methodik beschriebenen Atlanten vorgenommen.

Zur präzisen Bestimmung der unregelmäßigen Kontur des Hirnareals wurde neben dem manuellen Umfahren der Region im Programm MRICroN zusätzlich jeder für jede dazugehörige kleine Volumeneinheit (Voxel) einzeln korrigiert. Dies verhinderte eine versehentliche Mitmarkierung benachbarter Hirnstrukturen.

Viele frühere Studien verwendeten Markierungen nicht in jeder Schicht der MRTAufnahmen und errechneten dann das Volumen mit diesen 'Lücken'. Dies beschränkt die Genauigkeit der Messung. Um ein möglichst genaues Volumen messen zu können, wählten wir möglichst dünne Schichten (1 mm Schichtdicke). 
Außerdem kann es bei MRT-Aufnahmen zu Bewegungsartefakten und Unschärfen kommen. In der hiesigen Arbeit wurden eindeutig verzerrte und unscharfe MRTs bereits zu Beginn aussortiert und nicht vermessen.

Die Genauigkeit der Messungen hängt zudem stark von der individuellen Genauigkeit der messenden Person ab. Zur Überprüfung einer reproduzierbaren Grenzziehung und der Genauigkeit der Messungen berechneten wir die Retest(Messung durch ein und dieselbe Person von gleichen Patientendaten, welche zuvor verblindet wurden) und die Interrater-Reliabilität (Messung der gleichen Patientendaten durch von einander unabhängige Personen). Die Höhe der Reliabilität (Messgenauigkeit) sollte mindestens bei einer ICC $>0,85$ liegen. Der Retest war mit ICC $>0,99$ sehr hoch und auch die Interrater-Reliabilität war mit 0,960 sehr gut. Aufgrund der hohen individuellen Variabilität der Patientendaten war eine eindeutige Grenzziehung nicht immer anhand der vorher festgelegten anatomischen Strukturen zu realisieren, jedoch spricht die hohe Reliabilität der Messungen insgesamt für eine hohe Vergleichbarkeit und einen geringen Messfehler.

Die Messung der grauen und weißen Substanz wurde in unserer Studie getrennt voneinander durchgeführt. Da sich die Messungen der weißen Substanz als nicht reproduzierbar erwiesen, konzentrierten wir uns auf Messungen der grauen Substanz und verglichen nur diese Ergebnisse.

In den genannten Studien der MR-Volumetrie kortikaler Gyri, wurde zum Teil nur graue Substanz, zum Teil auch weiße Substanz in die Messungen einbezogen. Deswegen war es nicht möglich, Studien in der die graue Substanz untersucht wurde, mit Studien, die graue und weiße Substanz zusammen ermittelten, zu vergleichen.

Davon abgesehen ist der Vergleich von MRT- mit früheren postmortalen Messungen auf Grund der unterschiedlichen Gewebe- und Flüssigkeitsvolumina schwierig.

Unser Patientenkollektiv bestand aus 37 Kontrollen, 35 ersterkrankten Schizophreniepatienten, 39 Patienten mit bipolarer Störung und 27 Zwangspatienten. 
Alle Patienten stammten aus dem ambulanten und stationären Patientenkollektiv der Klinik für Psychiatrie und Psychotherapie des Universitätsklinikums des Saarlandes.

Entgegen unserer Hypothese der Reduktion des DLPFC-Volumens der grauen Substanz bei schizophren Ersterkrankten stellten wir ein signifikant höheres relatives DLPFC-Volumen (bezogen auf das Gesamtvolumen des präfrontalen Kortex, PFCGS-Volumen) fest.

Mögliche Ursache für das Ergebnis unserer Studie (signifikant vergrößertes Volumen der grauen Substanz des DLPFC relativ zur grauen Substanz des PFC bei Schizophreniepatienten im Vergleich zu gesunden Kontrollen) ist die Auswahl des Patientenkollektivs. Bei der Gruppe der schizophren Erkrankten handelte es sich um ersterkrankte Schizophreniepatienten zum Zeitpunkt der Aufnahme in die stationäre Behandlung. Eine mögliche Erklärung für das eher vergrößerte Volumen der Schizophreniepatienten in unserer Studie könnte die Tatsache sein, dass hirnmorphologische Veränderungen (also Volumenreduktionen) während des frühen Verlaufs der Erkrankung noch nicht nachgewiesen werden können, da diese möglicherweise erst bei einem längeren Krankheitsverlauf zu Tage treten. Die Ergebnisse könnten demnach in einigen Jahren anders ausfallen, obwohl dieselbe Methodik verwendet und dieselben Patienten untersucht werden würden.

Es liegen bis heute sehr wenige Studien vor, welche die Entwicklung der Hirnmorphologie während der Erkrankung beschreiben (z.B. im Rahmen einer Verlaufsuntersuchung). Weiterhin liegen kaum MR-volumetrische Studien vor, die ersterkrankte mit chronisch schizophrenen Patienten vergleichen. Einige Autoren vermuteten eine zunehmende Veränderung, zunächst hippocampal bei Ersterkrankten bis zur Volumenreduktion in Amygdala und kortikaler grauer Substanz bei chronisch Schizophrenen. In einer Studie zum Vergleich von Ersterkrankten und chronisch Schizophrenen fand man zwar keinen Hinweis eines Krankheitsfortschritts vom Hippocampus zur Amygdala, wohl aber eine Zunahme der kortikalen Veränderungen (Ellison-Wright et al. 2008).

Weitere Erklärungen für den in unserer Studie abweichenden Befund eines eher großen dorsolateralen präfrontalen Kortex bei schizophren Ersterkrankten könnte ein 
Sampleartefakt darstellen, d.h. in der zufälligen Stichprobe sind überdurchschnittlich große Gesamthirnvolumina bei diesen Patienten zu finden.

Außerdem war der Anteil an männlichen Probanden in dieser Gruppe sehr hoch (25 Männer/10 Frauen) und das Durchschnittsalter sehr jung ( $m=28,75 J a h r e)$. Damit sind in diesem Sample der schizophren Erkrankten zwei Charakteristika gegeben, die verhältnismäßig große Hirnvolumina unabhängig von einer Erkrankung im Vergleich zu den anderen Gruppen bedingen. Auch dies könnte mögliche Ursache für unser Ergebnis sein, obwohl beide Variablen zum Teil ais Kovariaten in die Auswertung einbezogen wurden.

Allerdings ergaben sich auch im matched Sample bei schizophren Ersterkrankten versus Kontrollen signifikant höhere DLPFC-Volumina der grauen Substanz relativ zum PFC-GS-Volumen. Dieses Sample war nach Alter, Bildungsdauer, Geschlecht und Händigkeit weitestgehend den gesunden Kontrollen angeglichen. Auf Grund der Gesamtstichprobenstruktur war allerdings nur ein ausreichendes Matching möglich, d.h. bei den Schizophrenen war weiterhin der Anteil der männlichen Probanden etwas höher, sie waren im Mittel etwas jünger und die Bildungsdauer war etwas kürzer als bei den Kontrollen. Alle diese Differenzen waren jedoch nach dem Matching nicht mehr signifikant.

Das absolute DLPFC-Volumen und das DLPFC-Volumen relativ zum gesamten GSVolumen war im matched Sample nicht erhöht.

Unser Ergebnis kontrastiert mit den Befunden in der Literatur. In zahlreichen Publikationen wurde über Volumenreduktionen berichtet. Woodruff et al. (1997) berichten über Volumenreduktionen im Frontal- und Temporallappen. Zu dem gleichen Ergebnis kommt auch Nopoulos et al. (1999). Sie untersuchten die vier Hirnlappen und das Cerebellum. Die Patienten wiesen kleinere Frontal- und Temporallappen auf, für das Cerebellum ergaben sich keine Gruppenunterschiede.

Goldstein et al. (1999) fanden signifikante Unterschiede bei Schizophrenen im Vergleich zu Kontrollen unter anderem im Bereich des Frontallappen. Dabei war das 
Volumen des Gyrus frontalis medius links ca. 8,8\%, das Volumen des frontomedialen Kortex beidseits um ca. 14,9\% und des orbitofrontalen Kortex rechts um ca. 8,5\% kleiner bei den Patienten im Vergleich zu den gesunden Kontrollen.

Auch Studien, die den Präfrontallappen in kleinere Untereinheiten aufteilten, konnten eine Abnahme des Gesamtvolumens des präfrontalen Kortex ausmachen. Sie zeigten auch verminderte Volumina für die graue Substanz in den Unterregionen (Buchanan et al. 1998, Bryant et al. 1999).

Sanfilipo et al. (2002) schilderten eine Reduktion der grauen Substanz im Präfrontallappen bei medizierten schizophrenen Patienten, welche mit schlechteren kognitiven Fähigkeiten korrelierte (psychomotorische Geschwindigkeit, kognitive Flexibilität und Sprachgewandtheit).

Auch andere Autoren schilderten eine Reduktion der grauen Substanz im Präfrontallappen bereits zum Zeitpunkt des Ausbruchs der Krankheit (Hirayasu et al. 2001, Lim et al. 1996, Nopoulos et al. 1995, Zipursky et al. 1998). Gur et al. (1999) konnten eine Verschlechterung neurokognitiver Leistungen einschließlich Abstraktionsvermögen, Aufmerksamkeit, verbales und räumliches Gedächtnis bei Abnahme des Volumens der grauen Substanz nachweisen.

Pomarol-Clotet et al. (2010) untersuchten in verschiedenen bildgebenden Verfahren die Lokalisation von Veränderungen der Hirnstrukturen bei Schizophreniepatienten. Sie benutzten voxel-basierte Morphometrie (VBM), funktionelle Magnetresonanztomographie und die Diffusions-Tensor-Bildgebung. Ihr Patientenkollektiv bestand aus 32 chronisch Schizophrenen und 32 gesunden Kontrollen.

Alle drei bildgebenden Verfahren zeigten eine Abweichung bei den Patienten im medialen frontalen Kortex im Vergleich zu den gesunden Kontrollen. In der VBM zeigte sich eine Volumenminderung in einer frontalen Hirnregion, die partiell auch den rechten DLPFC umfasst.

An strukturellen MRTs untersuchten Schobel et al. (2009) an Schizophrenie erkrankte Patienten und gesunde Kontrollen. Sie untersuchten den anterioren und posterioren Hippocampus, den orbitofrontalen Kortex und den DLPFC. An MRTs 
verglichen sie Strukturen und Volumina von hippokampalen und präfrontalen Subregionen und führten neurokognitive Tests bei Schizophreniepatienten durch. Anschließend wurde überprüft, ob es eine Assoziation von veränderten Hirnstrukturen zur Neurokognition gibt. Reduktionen wurden besonders im linken anterioren Hippocampus und im linken orbitofrontalen Kortex gefunden. Der DLPFC und der posteriore Hippocampus waren nicht signifikant verkleinert. Auch konnte in diesen beiden Abschnitten keine Beziehung zu neurokognitiven Defiziten festgestellt werden.

Kawada et al. (2009) hingegen konnten verminderte DLPFC-Volumina bei Schizophreniepatienten, im Vergleich zu gesunden Kontrollen zeigen. Ihre Untersuchungen fanden anhand der voxel-basierten Morphometrie statt. Des Weiteren fanden sie einen Zusammenhang zwischen reduzierten Volumina des DLPFC mit eingeschränkten frontalen Funktionen. Diese wurden mittels der „frontal system behaviour scale" ermittelt.

Schlaepfer et al. (1994) hielten eine unproportionale Abnahme der grauen Substanz im heteromodalen Assoziationskortex für spezifisch für an Schizophrenie erkrankte Patienten. Zum heteromodalen Assoziationskortex zählten sie den dorsolateralen präfrontalen Kortex, den inferioren parietalen Kortex und den superioren temporalen Gyrus. Sie untersuchten die graue Substanz des ganzen Gehirns und den spezifischen Regionen an transaxialen MRT-Aufnahmen. Untersucht wurden die Gehirne von schizophren Erkrankten, Patienten mit bipolarer Störung und gesunden Kontrollen. Mittels der Methode nach Harris et al. (1991) wurden halbautomatisch die folgenden Regionen vermessen: der DLPFC, der inferiore parietale Kortex und der superiore Temporallappen. Im Subtraktionsverfahren werden bei dieser Methode T2 gewichtete MRT-Bilder genutzt, um den Liquor cerebrospinalis (CSF) heller darzustellen. Halbautomatisch wurde nun auf den einzelnen Horizontalschichten die graue und weiße Substanz für die interessierende Region (ROI) markiert und das Gesamtvolumen aller drei Kortexregionen angegeben bzw. verglichen. Die Grenzen der Regionen wurden anhand der Schnitte festgelegt: DLPFC: vierter bis siebter Schnitt von der untersten bis vordersten kortikalen Region des zweiten Schnitts. Cortex parietalis inferior: sechster bis siebter von der untersten bis vordersten 
kortikalen Region des vierten Schnitts. Gyrus temporalis superior: unterste kortikale Region des zweiten Schnitts bis vorderste kortikale Region des dritten Schnitts.

Es konnte eine signifikante Volumenminderung für diese drei Regionen zusammengenommen festgestellt werden.

Im Gegensatz zu unserer Studie beschränkten sich Schlaepfer et al. (1994) beim Auswerten der MRTs auf bestimmte Schnitte. Individuelle Besonderheiten der Hirnanatomie konnten somit nicht berücksichtigt werden. Wir hingegen untersuchten nur eine dieser drei Substrukturen und legten die Grenzziehung des DLPFC auf dem Gyrus frontalis superior und den Gyrus frontalis medialis fest.

Eine mögliche Ursache für die gegensätzlichen Ergebnisse könnte also in der Festlegung der anatomischen Grenzen des DLPFC liegen. Wie im Methodikkapitel bereits beschrieben, orientierten wir uns bei der Grenzziehung an Crespo-Facorro et al. (1999) und Falkai et al. (2004). Crespo-Facorro et al. (1999) legen als dorsale Grenze des Präfrontalkortex das innere Knie des Corpus callosum fest. Falkai et al. (2004) hingegen beginnen mit ihren Messungen des Präfrontalkortex am äußeren Knie des Corpus callosum, da sie hier die dorsale Grenze des Präfrontalkortex festlegten.

Im Gegensatz zu Schlaepfer et al. (1994) legten Falkai et al. (2004) ihren Forschungsschwerpunkt auf die Form des Präfrontallappens. Sie untersuchten die Gestalt der präfrontalen und parieto-okzipitalen Region. Ihr Patientenkollektiv bestand aus 45 Familienangehörigen schizophrener Patienten, 27 Familienangehörigen mit anderen psychischen Erkrankungen und 51 Familienangehörigen ohne psychiatrische Erkrankungen nach ICD-10. Die Kontrollgruppe bestand aus 41 gesunden Personen ohne jegliche psychiatrische Erkrankung. An koronaren Schnitten von MRT-Aufnahmen wurden, ausgehend vom vorderen Knie des Corpus callosum als dorsale Grenze der präfrontalen und parietookzipitalen Region, Breite, Höhe und Länge der Regionen bestimmt. Der Quotient dieser Abmessungen gleicht einem Kreis wenn der Quotient sich 1 nähert.

Bei allen Familienangehörigen schizophrener Patienten war die Höhe der Präfrontalregion erhöht. Die Quotienten Breite/Höhe und Höhe/2x Länge näherten sich in Schizophrenie betroffenen Familien stärker 1 als in der Kontrollgruppe. Die 
Form des Präfrontalkortex näherte sich also einem Kreis in den koronaren Schnitten. In sagittalen Schnitten somit einem Halbkreis. In der parieto-okzipitalen Region gab es keine signifikanten Unterschiede.

Nach Falkai et al. (2004) liegt also eine Veränderung der Form (Stauchung) der präfrontalen Region bei Personen mit einer Disposition für Schizophrenie vor.

Da sich die Präfrontalregion in der Gehirnentwicklung erst spät entwickelt und genetischen Mechanismen unterliegt, geht man von einer Dysregulation bei Schizophrenie- und Risikopatienten aus. Das Präfrontalhirn ist in seiner Form also kürzer und höher als bei gesunden Kontrollen und wird somit gestaucht.

Wir fanden ein signifikant vergrößertes Volumen der grauen Substanz des DLPFC relativ zur grauen Substanz des PFC bei schizophrenen Patienten im Vergleich zu gesunden Kontrollen. Dieses Ergebnis könnte sich anhand der Studie von Falkai et al. (2004) erklären lassen. Durch die Stauchung des Gyrus frontalis superior und des Gyrus frontalis medialis könnte es zu einem größeren Volumen in unserem gemessenen Abschnitt kommen, dementsprechend könnte der orbitofrontale Kortex dagegen vermindert sein. Um den Aspekt der familiären Belastung zu berücksichtigen wurden die volumetrischen Ergebnisse zwischen der Gruppe der Ersterkrankten mit einem schizophrenen Angehörigen ersten oder zweiten Grades und den schizophren Erkrankten ohne familiäre Belastung verglichen. Auch hier fanden sich keine signifikanten Unterschiede.

Die Ergebnisse der MR-volumetrischen Studien in der Literatur finden überwiegend ihr Korrelat in den postmortalen Untersuchungen. So konnte beispielsweise in einer postmortalen Studie von Selemon et al. (2002) die Volumenreduktion im Frontallappen bestätigt werden.

\subsection{Bewertung des Einflusses intervenierender Variablen}

Wir fanden signifikante Geschlechtsdifferenzen für das GS- und PFC-GS Volumen und für das absolute DLPFC-Volumen. Beide waren bei den weiblichen Patienten signifikant kleiner als bei den männlichen Probanden $(p<0,006)$, was sowohl den Erfahrungen in der Literatur an Gesunden als auch Erkrankten entspricht. Für das relative DLPFC-Volumen ergaben sich keine signifikanten Geschlechtsdifferenzen, 
so dass sich hier globale Volumenreduktionen und keine regionalen Unterschiede abbilden.

Es ergaben sich signifikante Altersdifferenzen, Bipolare Patienten waren am ältesten, gefolgt von Zwangspatienten und Kontrollen, schizophren Ersterkrankte waren am jüngsten. Laut Falkai (2003) erkranken zwei Drittel der Schizophreniepatienten bereits vor dem 30. Lebensjahr. Männer erkranken etwa 3-4 Jahre früher als Frauen. Diese Erkenntnisse spiegeln sich in unserer Patientengruppe der schizophren Ersterkrankten wider. Die Verteilung Männer/Frauen lag bei 25/10 und das Durchschnittsalter betrug 28,75 Jahre.

Weiter fanden wir im Gesamtsample eine signifikant negative Korrelation zwischen dem Alter und dem gesamten Volumen der grauen Substanz, dem PFC-GS-Volumen und den absoluten DLPFC-Volumina.

Dies bedeutet eine Abnahme der dorsolateralen und gesamten grauen Substanz mit dem Alter. Ebenso signifikant negativ korrelierte das Alter mit den relativen DLPFCVolumen (bezogen auf die gesamte graue Substanz), d.h. der Alterseffekt bildet sich auch dann ab, wenn für die gesamte graue Substanz „normalisiert“ wird, ist also vermutlich in der Region DLPFC besonders ausgeprägt. Jackson et al. (2009) fanden auch eine Reduktion des Hirnvolumens im Alterungsprozess. Besonders stellten sie diese in präfrontalen und mittleren temporalen Regionen fest.

Die Auswertung der Faktoren Diagnose und Händigkeit führte zu folgendem Ergebnis. Während sich bei rechtshändigen Probanden im Mittel fast symmetrische, d.h. gleich große DLPFC-GS-Volumina auf beiden Seiten ergaben, zeigte sich bei den nicht-rechtshändigen Probanden eine rechtsseitige Asymmetrie, d.h. das DLPFC-GS-Volumen war im Durchschnitt rechts größer als links $(p=0,020)$.

Schaut man sich die Subgruppen numerisch an, so findet sich dieser beschriebene Unterschied nicht bei den schizophrenen Patienten.

Diesen Trend zur Aufhebung der Asymmetrie bei Schizophreniepatienten fanden auch Petty et al. (1995). In einer strukturell bildgebenden Studie wiesen sie den Verlust der normalen Asymmetrie am Planum temporale nach. Dieser Effekt konnte auch in einer postmortalen-Studie von Falkai et al. (1995) nachgewiesen werden. 
Des Weiteren konnte ein signifikanter Unterschied der Bildungsdauer zwischen den Patientengruppen festgestellt werden. Die längste Bildungsdauer wiesen Kontrollen, die kürzeste schizophrene Patienten auf. Im Gesamtsample korrelierte die Bildungsdauer signifikant positiv mit dem Volumen der gesamten grauen Substanz, d.h. ein größeres Volumen der grauen Substanz ging im Durchschnitt mit einer höheren Bildungsdauer (Bildungsstand) einher. Dies fanden auch Reiss et al. (1996), sie untersuchten gesunde Kinder und fanden eine positive Korrelation zwischen der Größe einiger zerebraler Strukturen, vor allem der grauen Substanz im Präfrontallappen, mit dem Intelligenzquotienten.

Allerdings ergab sich im Gesamtsample in der hiesigen Arbeit eine signifikant negative Korrelation zwischen der Schulbildung und dem relativen Volumen des DLPFC bezogen auf das graue Volumen des Präfrontalkortex (DLPFC-GS-Volumen relativ zu dem PFC-GS-Volumen), was damit zusammenhängen kann, dass die jüngeren Patienten (ersterkrankte Schizophrene) die niedrigste Schulbildung aufwiesen. Für die Kontrollgruppe alleine, korrelierte die Bildungsdauer (wie im Gesamtsample) signifikant positiv mit dem Volumen der gesamten grauen Substanz. Hier ergab sich zusätzlich eine positive Korrelation von Bildungsdauer und der grauen Substanz des präfrontalen Kortex (PFC-GS-Volumen).

\subsection{Bewertung der Subgruppenvergleiche}

Die Diagnose übergreifenden Analysen galten der Fragestellung, ob es eine Krankheitsspezifität für Volumenveränderung des dorsolateralen präfrontalen Kortex gibt. Eine solche Spezifität konnten anhand der Daten in dieser Arbeit nicht gezeigt werden. In den Gruppen 'Bipolare Patienten' und 'Zwangspatienten' konnten keine signifikanten Volumenunterschiede des DLPFC im Vergleich zu gesunden Kontrollen gezeigt werden. 


\subsection{Ausblick}

Nach intensiver Betrachtung der Ergebnisse unserer Studie treten neue Fragestellungen in den Vordergrund. Wie oben beschrieben könnte die Tatsache, dass wir schizophren Ersterkrankte untersuchten, ursächlich für unser Ergebnis sein. Anschließende Studien könnten demnach von folgenden Fragen geleitet sein:

1 Besteht ein Volumenunterschied des DLPFC bei schizophren Ersterkrankten im Vergleich zu chronisch Schizophrenen?

$2 \mathrm{Ab}$ welcher Dauer der Erkrankung ist ein Volumenunterschied messbar?

Auch könnte die Veränderung der Form des Präfrontalkortex (Stauchung) nach Falkai et al. (2004) Ursache für unser Studienergebnis sein. Bislang weiß man von der Stauchung des gesamten Präfrontalkortex. Nicht geklärt ist jedoch, ob die Stauchung gleichmäßig verteilt ist oder ob etwa die orbitofrontale Region stärker gestaucht sein könnte als der dorsale Präfrontalkortex. Unser Studienergebnis ermutigt also zur Untersuchung mehrerer Regionen des präfrontalen Kortex, um hier genauere Aussagen machen zu können.

Es bleiben also interessante Fragestellungen offen, die Forschungsziel weiterer Studien darstellen können. 


\section{$5 \quad$ Zusammenfassung}

Dem dorsolateralen präfrontalen Kortex (DLPFC) wird, als Teil des Präfrontalkortex, die Lokalisation des Arbeitsgedächtnisses zugeschrieben. Bei schizophrenen Patienten konnten in neuropsychologischen Studien regelmäßig Defizite bei Tests festgestellt werden, die sogenannte exekutive Funktionen erfassen. Dem Arbeitsgedächtnis als Vermittler exekutiver Funktionen kommt dabei eine besondere Bedeutung zu. Dieses System ist zuständig für die vorübergehende Speicherung und gleichzeitige Verarbeitung von Informationen und ist bei Schizophrenen besonders häufig beeinträchtigt.

Die vorliegende Arbeit beschäftigt sich mit der volumetrischen Untersuchung des DLPFC auf MRT-Aufnahmen. Untersucht wurden verschiedene Krankheitsbilder. Das Patientenkollektiv bestand aus 35 schizophren Ersterkrankten, 39 bipolar Erkrankten, 27 Zwangspatienten und 37 Kontrollen. Fragestellung der Arbeit war, ob ein Unterschied (Verminderung) der Volumina des DLPFC bei Schizophrenie im Vergleich zu einer gesunden Kontrollgruppe besteht. Des Weiteren beschäftigte uns die Frage, ob ein eventueller Unterschied (Reduktion) diagnosespezifisch ist. Hierzu wurde der Vergleich zu einer Gruppe von Bipolaren Patienten und Zwangspatienten herangezogen.

Gemessen wurde die graue Substanz des DLPFC beider Hirnhälften in den zuvor festgelegten Grenzen. Die Volumetrie erfolgte mit dem Programm MRICroN (Chris Rorden) auf T1-gewichteten MR-Aufnahmen (1,5 Tesla, 1cmm Voxel) nach der Volume-of-Interest-Methode mit manuellem Tracing. Die Wiederholungsmessungen an 10 Probanden erbrachten sehr gute Retest- und Interrater-Reliabilitäten mit einer Intraclass-Korrelation von über 0,95.

Bei der statistischen Auswertung ergab sich ein signifikant vergrößertes Volumen der grauen Substanz des DLPFC relativ zur grauen Substanz des PFC bei den schizophrenen Patienten im Vergleich zu gesunden Kontrollen. Die Bipolaren und Zwangspatienten zeigten keine signifikanten Volumenunterschiede zu den gesunden Kontrollen. Die in der Literatur beschriebene Volumenminderung des Präfrontalkortex sowie des DLPFC bei an Schizophrenie Erkrankten ließ sich somit an unserem Sample mit der von uns verwendeten manuellen Methode nicht replizieren. 
Verantwortlich hierfür könnten zum einen Charakteristika unserer Stichproben sein, zum anderen die hier verwendete manuelle Messmethode. Möglicherweise lässt sich eine Verminderung des Volumens des DLPFC erst nach mehrjähriger Krankheit darstellen und noch nicht bei der Gruppe der ersterkrankten schizophrenen Patienten. Ebenso ursächlich für das gefundene erhöhte DLPFC-Volumen bei den Schizophreniekranken könnte eine Stauchung des Präfrontalhirns und damit eine Formveränderung mit konsekutiver Volumenminderung anderer, mehr anterior gelegener Präfrontalregionen sein. 


\section{Summary}

The presented doctoral thesis deals with the MR-volumetric measurement of the dorsolateral prefrontal cortex (DLPFC) of patients suffering from different psychiatric diseases. In schizophrenia patients several neuropsychological studies demonstrated cognitive deficits in executive functioning. Working memory is seen as a essential part of executive functions. The function of working memory is characterised by the temporarily storing and handling of information which is often disturbed in schizophrenia patients.

The MR sample consists of 35 first-episode schizophrenia patients, 39 bipolar patients, 27 patients with obsessive-compulsive disorder and 37 healthy control subjects. With this study the issue was addressed if there is a volumetric difference of the DLPFC between first-episode schizophrenia patients and healthy subjects.

Further we explored if possible volumetric differences can also be shown in schizophrenia patients compared to bipolar patients and patients with obsessivecompulsive disorder.

The DLPFC volumes have been measured on both sides to discover asymmetries. The volumetric measurements were performed by using the program MRIcroN with the volume of interest approach (manually tracing DLPFC) on T1-weighted MR scans (1,5 Tesla, $1 \mathrm{cmm}$ voxel). The repeated measurements of $10 \mathrm{MR}$ scans (10 subjects) provided high retest and interrater reliability showing an intraclass correlation of more than 0.95 .

Statistical analysis showed significant differences between the groups regarding the relative grey matter DLPFC volume (DLPFC volume in relation to the grey matter prefrontal cortex volume (PFC). Relative DLPFC volume was increased in firstepisode patients compared to healthy controls. Bipolar patients and patients with obsessive-compulsive disorder demonstrated no differences compared to healthy controls. In conclusion, with our volumetric approach in this study no disease-related DLPFC volume reduction could be observed in schizophrenia patients, as it has been reported several times in the literature. This may be related to the characteristics of our study sample and the used morphometric methods. Volume reduction in schizophrenia may occur during the later course due to the disease progression but 
not already in first-episode schizophrenia. Furthermore a change in prefrontal cortex shape (compression of anterior frontal cortex parts) could be responsible for our result of increased DLPFC with consecutive volume reduction in other prefrontal areas. 


\section{$7 \quad$ Verzeichnisse}

\subsection{Tabellenverzeichnis}

Tabelle 1: $\quad$ Risikofaktoren der Schizophrenie (Falkai 2003, 17) S. 8

Tabelle 2: Soziodemografische und klinische Variablen der ersterkrankten Schizophrenen

Tabelle 3: Soziodemografische und klinische Variablen der ersterkrankten Schizophrenen (Fortsetzung) S.16

Tabelle 4: Soziodemografische Variablen der Bipolaren Patienten $\quad$ S.17

Tabelle 5: Soziodemografische Variablen der Zwangspatienten S.18

Tabelle 6: Soziodemografische Variablen der gesunden Kontrollgruppe $\quad$ S.19

Tabelle 7: Berechnung der Intrarater-Reliabilität $\quad$ S.28

Tabelle 8: Berechnung der Interrater-Reliabilität $\quad$ S.29

Tabelle 9: Darstellung und Vergleich der soziodemografischen und klinischen Variablen der MR-Stichproben

Tabelle 10: Darstellung und Vergleich der soziodemografischen und klinischen Variablen der MR-Stichproben (Fortsetzung)

Tabelle 11: Vergleich der Hirnvolumina in den Diagnosegruppen

Tabelle 12: Vergleich der Hirnvolumina-Subgruppenvergleich-Analysen (Bonferroni)

Tabelle 13: Einfluss von intervenierenden Variablen und Darstellung der korrigierten p-Werte nach Bonferroni-Korrektur in der Gesamt- und Subgruppen-Analyse

Tabelle 14: Vergleich der abhängigen Variablen zwischen Kontrollen und schizophren ersterkrankten Patienten im gematchten Sample

Tabelle 15: Einfluss familiärer Belastung bei ersterkrankten Schizophreniepatienten

Tabelle 16: Schizophren ersterkrankte: Partielle Korrelationen zwischen Krankheitsverlaufsdaten und Psychopathologie

Tabelle 17: Schizophren ersterkrankte: Partielle Korrelationen zwischen Krankheitsverlaufsdaten und Psychopathologie (Fortsetzung) 
Tabelle 18: Ergebnisse neuerer volumetrischer MR-Studien bezüglich des präfrontalen Kortex bei ersterkrankten Schizophrenen S.53 


\subsection{Abbildungsverzeichnis}

Abbildung 1 Schematische Darstellung von Subregionen

des Stirnhirns (Förstl 2004, 22)

S.10

Abbildung 2

Markierung des DLPFC in der Sagittalebene

(T1-gewichtetes MR-Schnittbild)

S.23

Abbildung 3 Markierung des DLPFC in der Koronarebene (T1-gewichtetes MR-Schnittbild)

Abbildung 4 Markierung des DLPFC in der Transversalebene (T1-gewichtetes MR-Schnittbild) 


\subsection{Literaturverzeichnis}

(1) Andreasen NC, Black DW: Diagnostic and statistical manual of mental disorders, Beltz Verlag, Weinheim 1993

(2) Andreasen NC, Rezai K, Alliger R, Swayze VW 2nd, Flaum M, Kirchner P, Cohen G, O`Leary DS (1992): Hypofrontality in neuroleptic- naive patients and in patients with chronic schizophrenia. Assessment with xenon 133 single-photon emission computed tomography and the Tower of London. Arch Gen Psychiatry $\underline{49}$ (12), 943-958

(3) Bachneff SA (1991): Positron Emission Tomography and Magnetic Resonance Imaging: a review and a local circuit neurons hypo(dys)function hypothesis of schizophrenia. Biol Psychiatry 30 (9), 857-886

(4) Baddeley A (1992): Working memory. Science 255 (5044), 556-559

(5) Benes FM, McSparren J, Bird ED, SanGiovanni JP, Vincent SL (1991): Deficits in small interneurons in prefrontal and anterior cingulate cortex of schizophrenic and schizoaffective patients. Arch Gen Psychiatry $\underline{48}$ (11), 996-1001

(6) Berman KF, Illowsky BP, Weinberger DR (1988): Physiological dysfunction of dorsolateral prefrontal cortex in schizophrenia .IV. Further evidence for regional and behavioral specificity. Arch Gen Psychiatry $\underline{45}$ (7), 616- 622

(7) Bestehehorn M, Tischer B, Glaser P, Mast O, Schmidt D (1999):

Repräsentative Studie zur Verteilung schizophrener Patienten auf medizinische Versorgungseinrichtungen in Deutschland. Fortschr Neurol Psychiatr $\underline{67}$, 487-492

(8) Bleuler E: Dementia praecox oder Gruppe der Schizophrenien. In: Handbuch der Psychiatrie.hrsg: Aschaffenburg G, Deuticke, Leipzig 1911, 1-8

(9) Bonnici HM, William T, Moorhead TW, Stanfield AC, Harris JM, Owens DG, Johnstone EC, Lawrie SM (2007): Pre-frontal lobe gyrification index in schizophrenia, mental retardation and comorbid groups: an automated study. Neuroimage $\underline{35}$ (2), 648-654 
(10) Brodmann K: Vergleichende Lokalisationslehre der Großhirnrinde in ihren Prinzipien dargestellt auf Grund des Zellenaufbaus, Barth, Leipzig 1909

(11) Bryant NL, Buchanan RW, Vladar K, Breier A, Rothman M (1999): Gender differences in temporal lobe structures of patients with schizophrenia: $A$ volumetric study. Am J Psychiatry 156 (4), 603-609

(12) Bryson G, Bell M, Greig T, Kaplan T (1999): Internal consistency, temporal stability and neuropsychological correlates of three cognitive components of the Positive and Negative Syndrom Scale PANSS. Schizophr Res $\underline{38}$ (1), 27-35

(13) Buchanan RW, Vladar K, Barta PE, Pearlson GD (1998): Structural evaluation of the prefrontal cortex in schizophrenia. Am J Psychiatry 155 (8), 10491055

(14) Cannon TD (1997): On the nature and mechanisms of obstetric influences in schizophrenia: a review and synthesis of epidemiologic studies. Int Rev Psychiatry $\underline{9}, 387-397$

(15) Cardno AG, Gottesmann II (2000): Twin studies of schizophrenia: from bowand-arrow concordances to Star Wars Mx and functional genomics. Am J Med Genet $\underline{97}$ (1), 12-17

(16) Crespo-Facorro B, Kim JJ, Andreasen N, O`Leary D, Wiser A, Bailey J, Harris G, Magnotta VA (1999): Human Frontal Cortex: An MRI-based parcellation method. Neuroimage 10 (5), 500-519

(17) DeLisi LE, Sakuma M, Tew W, Kushner M, Hoff AL, Grimson R (1997): Schizophrenia as a chronic active brain process: a study of progressive brain structural change subsequent to the onset of schizophrenia. Psychiatry Res $\underline{74}$ (3), 129-140

(18) Dilling $\mathrm{H}$, Mombour $\mathrm{W}$, Schmidt $\mathrm{MH}$ (Hrsg.): Internationale Klassifikation psychischer Störungen. ICD-10-Kapitel V (F). Klinisch-diagnostische Leitlinien. Huber, Bern-Göttingen-Toronto 1999

(19) Duvernoy HM: The Human Brain; Surface, Blood Supply and ThreeDimensional Sectional Anatomy; Springer Wien, New York 1999 
(20) Elliott R, McKenna P, Robbins TW, Sahakian B (1995): Neuropsychological evidence for frontostriatal dysfunction in schizophrenia. Psychol Med $\underline{25}$ (3), 619-630

(21) Ellison-Wright I, Glahn DC, Laird AR, Thelen SM, Bullmore E (2008): The anatomy of first-episode and chronic schizophrenia: an anatomical likelihood estimation meta-analysis. Am J Psychiatry 165 (8), 1015-1023

(22) Falkai P: Schizophrenie auf einen Blick, Blackwell Verlag, Berlin 2003

(23) Falkai P, Bogerts B (1986): Cell loss in the hippocampus of schizophrenics. Eur Arch Psych Neurol Sci 236 (3), 154-161

(24) Falkai P, Bogerts B, Schneider T, Greve B, Pfeiffer U, Pilz K, Gonsiorzcyk C, Majtenyi C, Ovary I (1995): Disturbed planum temporal asymmetry in schizophrenia. A quantative post-mortem study. Schizophr Res 14 (2), 161-176

(25) Falkai P, Honer WG, Alfter D, Schneider-Axmann T, Bussfeld P, Cordes J, Blank B, Schönell H, Steinmetz H, Maier W, Tepest R (2002): The temporal lobe in schizophrenia from uni- and multiply affected families. Neurosci Lett $\underline{325}$, 25-28

(26) Falkai P, Tepest R, Honer WG, Dani I, Ahle G, Pfeiffer U, Vogeley K, Schulze TG, Rietschel M, Cordes J, Schönell H, Gaebel W, Kühn KU, Maier W, Träber F, Block W, Schild HH, Schneider-Axmann T (2004): Shape changes in prefrontal, but not parieto-occipital regions: brains of schizophrenic patients come closer to a circle in coronal and sagittal view. Psychiatry Res 132 (3), 261-271.

(27) Fananas L, Moral P, Bertranpetit J (1990): Quantitative dermatoglyphics in schizophrenia: study of family history subgroups. Hum Biol $\underline{62}$ (3), 421-427

(28) Farrow TF, Whitford TJ, Williams LM, Gomes L, Harris AW (2005): Diagnosisrelated regional gray matter loss over two years in first episode schizophrenia and bipolar disorder. Biol Psychiatry $\underline{58}$ (9), 713-723

(29) Fatemi SH, Emamian ES, Kist D, Sidwell RW, Nakajima K, Akhter P, Shier A, Sheikh S, Bailey K (1999): Defective corticogenesis and reduction in Reelin immunoreactivity in cortex and hippocampus of prenatally infected neonatal mice. Mol Psychiatry 4 (2), 145-154 
(30) Förstl H.: Frontalhirn, Springer Medizin Verlag Heidelberg 2004

(31) Fuster JM (1997): Network memory. Trends Neurosci 20 (10), 451-459

(32) Geddes JR, Verdoux H, Takei N, Lawrie SM, Bovet P, Eagles JM, Heun R, McCreadie RG, McNeil TF, O'Callaghan E, Stöber G, Willinger U, Murray RM (1999): Schizophrenia and complications of pregnancy and labour: an individual patient data meta-analysis. Schizophr Bull 25 (3), 413-423

(33) Goldman-Rakic PS: Circuittry of the primate prefrontal cortex and the regulation of bahaviour by representational knowledge. In: handbook of physiology, The Nervous System, Higher Functions of the Brain, hrsg.:Plum F, American Physiological Society, Bethesda/USA, 1987, 373417

(34) Goldman-Rakic PS (1992) Working memory and the mind. Sci Am 267 (3), 110-117

(35) Goldman-Rakic PS, Selemon LD (1997): Functional and anatomical aspects of prefrontal pathology in schizophrenia. Schizophr Res 23, 437-458

(36) Goldstein JM, Goodman JM, Seidman LJ, Kennedy DN, Makris N, Lee H, Tourville J, Caviness VS, Faraone SV, Tsuang MT (1999): Cortical abnormalities in schizophrenia identified by structural magnetic resonance imaging. Arch Gen Psychiatry $\underline{56}$ (6), 537-547

(37) Gothelf D, Frisch A, Munitz H, Rockah R, Laufer N, Mozes T, Hermesh H, Weizman A, Frydman M (1999): Clinical characteristics of schizophrenia associated with velo-cardio-facial syndrome. Schizophr Res $\underline{35}$ (2), 105112

(38) Gottesmann II, Bertelsen A (1989): Confirming unexpressed genotypes for schizophrenia. Risks in the offspring of Fischer's Danish identical and fraternal discordant twins. Arch Gen Psychiatry $\underline{46}$ (10), 867-872

(39) Gur RE, Turetsky BI, Bilker WB, Gur RC (1999): Reduced gray matter volume in schizophrenia. Arch Gen Psychiatry $\underline{56}$ (10), 905-911 
(40) Guy W (1976) (Ed.): Clinical Global Impressions (CGI) Scale. Modified from:

Rush et al.: Psychiatric Measures, APA, Washington DC 2000. Retrieved October 28, 2009 from

https://sakai.ohsu.edu/access/content/user/brodym/.../CGI.doc

(41) Häfner H (1988): Epidemiologie der Schizophrenie. Stand der Perspektiven, Fundamenta Psychiatrica 4 , 264-282

(42) Häfner $\mathrm{H}$, an der Heiden W, Hambrecht M, Riecher-Rössler A, Maurer K, Löffler W, Fätkenheuer B, Gupta S (1993): Ein Kapitel systematischer Schizophrenieforschung-Die Suche nach kausalen Erklärungen für den Geschlechtsunterschied im Ersterkrankungsalter. Nervenarzt 64, 706-716

(43) Harris GJ, Rhew EH, Noga T, Pearlson GD (1991): User-friendly method for rapid brain and CSF volume calculation using transaxial MRI images. Psychiatry Res $\underline{40}$ (1), 61-8

(44) Hirayasu Y, Tanaka S, Shenton ME, Salisbury DF, DeSantis MA, Levitt JJ, Wible C, Yurgelun- Todd D, Kinikis R, Jolesz FA, Mc Carley RW (2001): Prefrontal gray matter reduction in first episode schizophrenia. Cereb Cortex 11 (4), 374-381

(45) Jablensky A (2000): Epidemiology of schizophrenia: the global burden of disease and disability. Eur Arch Psychiatry Clin Neurosci 250 (6), 274-285

(46) Jackson J, Balota DA, Head D (2009): Exploring the relationship between personality and regional brain volume in healthy aging. Neurobiol Aging. Dezember 23, PMID: 20036035

(47) Kawada R, Yoshizumi M, Hirao K, Fujiwara H, Miyata J, Shimizu M, Namiki C, Sawamoto N, Fukuyama H, Hayashi T, Murai T (2009): Brain volume and dysexecutive behavior in schizophrenia. Prog Neuropsychopharmacol Biol Psychiatry $\underline{33}$ (7), 1255-1260

(48) Kay SR, Fiszbein A, Opler LA (1987): The positive and negative syndrome scale (PANSS) for schizophrenia. Schizophr Bull $\underline{13}$ (2), 261-276

(49) Kissling W, Höffler J, Seemann U, Müller P, Rüther E, Trenckmann U, Uber A, Graf v. d. Schulenburg JM, Glaser P, Glaser T, Mast O, Schmidt D (1999): 
Die direkten und indirekten Kosten der Schizophrenie. Fortschr Neurol Psychiatr $\underline{67}$ (1), 29-36

(50) Kraepelin E: Lehrbuch der Psychiatrie, 5. Auflage, Barth, Leipzig 1896

(51) Lawrie SM, Abukmeil SS (1998): Brain abnormalities in schizophrenia. A systematic and quantitative rewiew of volumetric magnetic resonance imaging studies. Br J Psychiatry 172,110-120

(52) Lim KO, Tew W, Kushner M, Chow K, Matsumoto B, DeLisi LE (1996): Cortical grey matter volume deficits in patients with first-episode schizophrenia. Am J Psychiatry 153 (12), 1548-1553

(53) Lopez-Garcia P, Aizenstein HJ, Snitz BE, Walter RP, Carter CS (2006): Automated ROI-based brain parcellation analysis of frontal and temporal brain volumes in schizophrenia. Psychiatry Res 147 (2-3):153-161

(54) Mai JK, Assheuer J, Paxinos G: Atlas of the Human Brain; Academic Press, San Diego 1997

(55) Maier W, Rietschel M, Lichtermann D, Wildenauer DB (1999): Family and genetic studies on the relationship of schizophrenia to affective disorders. Eur Arch Psychiatry Clin Neurosci 249 (4), 57-61

(56) Matthes-von Cramon G, Cramon Y: Störungen exekutiver Funktionen. In: Lehrbuch der Klinischen Neuropsychologie, Sturm W, Herrmann M, hrsg.:Wallesch C-W, Swets \& Zeitlinger, Lisse/NL 2000, 392-410

(57) McGrath JJ, Murray RM: Risk factors for schizophrenia: from conception to birth. In: Schizophrenia, Weinberger D, Hirsch SR, Blackwell Publishing, Oxford/UK 2003, 232-250

(58) Meisenzahl EM, Koutsouleris N, Bottlender R, Scheuerecker J, Jäger M, Teipel SJ, Holzinger S, Frodl T, Preuss U, Schmitt G, Burgermeister B, Reiser M, Born C, Möller HJ (2008): Structural brain alterations at different stages of schizophrenia: a voxel-based morphometric study. Schizophr Res 104 (1-3), 44-60

(59) Molina V, Sanz J, Sarramea F, Luque R, Benito C, Palomo T (2006): Dorsolateral prefrontal and superior temporal volume deficits in first- 
episode psychoses that evolve into schizophrenia. Eur Arch Psychiatry Clin Neurosci 256 (2), 106-111

(60) Montgomery SA, Asberg M (1979): A new depression scale designed to be sensitive to change. Br J Psychiatry 134, 382-389

(61) Nopoulos P, Torres L, Flaum M, Andreasen NC, Ehrhardt JC, Yuh WT (1995): Brain morphology in first-episode schizophrenia. Am J Psychiatry 152 (12) 1721-1723

(62) Nopoulos PC, Ceilley JW, Gailis EA, Andreasen NC (1999): An MRI study of cerebellar vermis morphology in patients with schizophrenia: evidence in support of the cognitive dysmetria concept. Biol Psychiatry 46 (5), 703-711

(63) Nuechterlein KH, Dawson ME, Ventura J, Gitlin M, Subotnik KL, Snyder KS, Mintz J, Bartzokis G (1994): The vulnerability/stress model of schizophrenic relapse: a longitudinal study. Acta Psychiatr Scand Suppl. 382, 58-64

(64) Pedersen CB, Mortensen PB (2001): Evidence of a dose-response relationship between urbanicity during upbringing and schizophrenia risk. Arch Gen Psychiatry $\underline{58}$ (11), 1039-1046

(65) Petty RG, Barta PE, Pearlson GD, McGilchrist IK, Lewis RW, Tien AY, Pulver A, Vaughn DD, Casanova MF, Powers RE (1995): Reversal of asymmetry of the planum temporal in schizophrenia. Am J Psychiatry 152 (5), 715721

(66) Pomarol-Clotet E, Canales-Rodríguez EJ, Salvador R, Sarró S, Gomar JJ, Vila F, Ortiz-Gil J, Iturria-Medina Y, Capdevila A, McKenna PJ (2010): Medial prefrontal cortex pathology in schizophrenia as revealed by convergent findings from multimodal imaging. Mol Psychiatry Januar 12, PMID: 20065955

(67) Reiss AL, Abrams MT, Singer HS, Ross JL, Denckla MB (1996): Brain development, gender and IQ in children. A volumetric imaging study. Brain $\underline{119}, 1763-1774$.

(68) Robbins TW (1990): The case for frontostriatal dysfunction in schizophrenia. Schizophr Bull 16 (3), 391-402 
(69) Sanfilipo M, Lafargue T, Rusinek H, Arena L, Loneragan C, Lautin A, Rotrosen J, Wolki A (2002): Cognitive performance in schizophrenia: relationship to regional brain volumes and psychiatric symptoms. Psychiatry Res 116 (1-2), 1-23

(70) Saß H, Wittchen HU, Zaudig M: Diagnostisches und Statistisches Manual Psychischer Störungen DSM-IV. Hogrefe, Göttingen 1996

(71) Schaufelberger MS, Duran FL, Lappin JM, Scazufca M, Amaro E Jr, Leite CC, de Castro CC, Murray RM, McGuire PK, Menezes PR, Busatto GF (2007): Grey matter abnormalities in Brazilians with first-episode psychosis. $\mathrm{Br} \mathrm{J}$ Psychiatry 191 (51), 117-122

(72) Schlaepfer TE, Harris GJ, Tien AY, Peng LW, Lee S, Federman EB, Chase GA, Barta PE, Pearlson GD (1994): Decreased regional cortical gray matter volume in schizophrenia. Am J Psychiatry $\underline{51}$ (6), 842-848

(73) Schmitt A, Weber-Fahr W, Jatzko A, Tost H, Henn FA, Braus DF (2001): Current overview of structural magnetic resonance imaging in schizophrenia. Fortschr Neurol Psychiatr $\underline{69}$ (3), 105-115

(74) Schobel SA, Kelly MA, Corcoran CM, Van Heertum K, Seckinger R, Goetz R, Harkavy-Friedman J, Malaspina D (2009): Anterior hippocampal and orbitofrontal cortical structural brain abnormalities in association with cognitive deficits in schizophrenia. Schizophr Res 114 (1-3), 110-118

(75) Selemon LD, Kleinman JE, Herman MM, Goldman-Rakic PS (2002): Smaller frontal gray matter volume in postmortem schizophrenic brains. Am J Psychiatry $\underline{159}$ (12), 1983-1991

(76) Silver H, Feldmann P, Bilker W, Gur RC (2003): Working memory deficit as a core neuropsychological dysfunction in schizophrenia. Am J Psychiatry $\underline{160}$ (10), 1809-1816

(77) Steen RG, Mull C, McClure R, Hamer RM, Lieberman JA (2006): Brain volume in first-episode schizophrenia. Br J Psychiatry 188, 510-518

(78) Takayanagi Y, Kawasaki Y, Nakamura K, Takahashi T, Orikabe L, Toyoda E, Mozue Y, Sato Y, Itokawa M, Yamasue H, Kasai K, Kurachi M, Okazaki Y, 
Matsushita M, Suzuki M (2010): Differentiation of first-episode schizophrenia patients from healthy controls using ROI-based multiple structural brain variables. Prog Neuropsychopharmacol Biol Psychiatry $\underline{34}$ (1), 10-17

(79) Talairach J, Tournoux P: Co-Planar Stereotaxic Atlas of the human Brain; 3Dimensional Proportional System: An Approach to Cerebral Imaging. Thieme, Stuttgart 1988

(80) Torrey EF, Miller J, Rawlings R, Yolken RH (1997): Seasonality of births in schizophrenia and bipolar disorder: a review of the literature. Schizophr Res $\underline{28}$ (1), 1-38

(81) Vogeley K, Tepest R, Pfeiffer U, Schneider-Axmann T, Maier W, Honer WG, Falkai P (2001): Right frontal hypergyria differentiates affected from nonaffected siblings in families with schizophrenia. A morphometric MRI study. Am J Psychiatry 158 (3), 494-496

(82) Weinberger D, Aloia M, Goldberg T, Berman K (1994): The frontal lobes and schizophrenia. J Neuropsychiatry Clin Neurosci $\underline{6}$ (4), 419-427

(83) Whitford TJ, Farrow TF, Gomes L, Brennan J, Harris AW, Williams LM (2005): Grey matter deficits and symptom profile in first episode schizophrenia. Psychiatry Res 139 (3), 229-238

(84) Whitford TJ, Farrow TF, Williams LM, Gomes L, Brennan J, Harris AW (2009): Delusions and dorso-medial frontal cortex volume in first-episode schizophrenia: a voxel-based morphometry study. Psychiatry Res $\underline{172}$ (3), 175-179

(85) Wobrock T, Pajonk FG, Falkai P (2004): Schizophrenie. Epidemiologie, Ätiopathogenese, Symptomatologie, Diagnostik und Differenzialdiagnostik. Fortschr Neurol Psychiatr $\underline{72}$, 98-113

(86) Woodruff PW, Wright IC, Shuriquie N, Russouw H, Rushe T, Howard RJ, Graves M, Bullmore ET, Murray RM (1997): Structural brain abnormalities in male schizophrenics reflect, fronto-temporal dissociation. Psychol Med $\underline{27}(6), 1257-1266$

(87) Young RC, Biggs JT, Ziegler VE, Meyer DA (1978): A rating scale for mania: reliability, validity and sensitivity. Br J Psychiatry $\underline{133}$, 429-435 
(88) Zipparo L, Whitford TJ, Redoblado Hodge MA, Lucas S, Farrow TF, Brennan J, Gomes L, Williams LM, Harris WF (2007): Investigating the neuropsychological and neuroanatomical changes that occur over the first 2-3 years in patients with first-episode schizophrenia. Prog Neuropsychopharmacol Biol Psychiatry $\underline{32}$ (2), 531-538

(89) Zipursky RB, Lambe EK, Kapur S, Mikulis DJ (1998): Cerebral gray matter volume deficits in first episode psychosis. Arch Gen Psychiatry $\underline{55}$ (6), $540-546$ 


\section{Danksagungen}

An erster Stelle geht mein Dank an Prof. Dr. med. P. Falkai (Direktor der Abteilung Psychiatrie und Psychotherapie, Universitätsklinikum Georg-August-Universität Göttingen) für die Überlassung des interessanten Themas.

Besonders bedanken möchte ich mich bei Herrn PD Dr. med. T. Wobrock (Oberarzt der Abteilung Psychiatrie und Psychotherapie, Universitätsklinikum Georg-AugustUniversität Göttingen), der mir bei der Planung, Durchführung und Abfassung der Arbeit eine unersetzbare Hilfe war.

Besonderer Dank gilt dem Statistiker Thomas Schneider-Axmann aus Bremen.

Außerdem danke ich dem Personal der Neuroradiologie in Homburg/Saar, das an der Entstehung der Bilder beteiligt war, sowie allen Patienten und Probanden, deren Aufnahmen mir zur Verfügung standen. 